\title{
A Facile and Efficient Synthesis of Polyfunctionalized Pyridin-2(1H)-ones from $\beta$-Oxo Amides under Vilsmeier Conditions
}

Dexuan Xiang, ${ }^{1}$ Kewei Wang, ${ }^{1}$ Yongjiu Liang, ${ }^{2}$ Guangyuan Zhou, ${ }^{2 *}$ and Dewen Dong ${ }^{1,2 *}$

1. Department of Chemistry, Northeast Normal University, Changchun, 130024, China.

2. Changchun Institute of Applied Chemistry, Chinese Academy of Sciences, Changchun, 130022, China.

\section{Supporting Information}

Table of contents

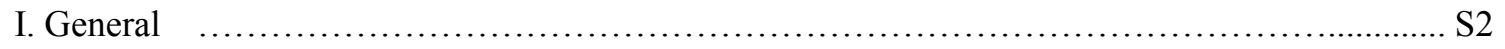

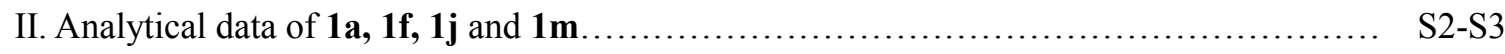

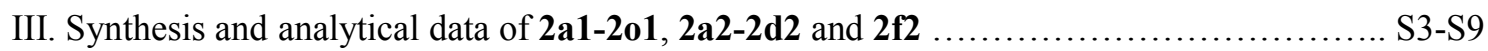

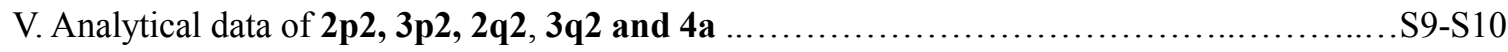

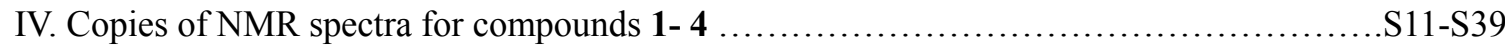




\section{I.General}

All reagents were purchased from commercial sources and used without treatment, unless otherwise indicated. The products were purified by column chromatography over silica gel. ${ }^{1} \mathrm{H}$ NMR and ${ }^{13} \mathrm{C}$ NMR spectra were recorded at $25^{\circ} \mathrm{C}$ at $500 \mathrm{MHz}$ and $125 \mathrm{MHz}$, respectively, with TMS as internal standard. IR spectra $(\mathrm{KBr})$ were recorded on FTIR-spectrophotometer in the range of $400-4000 \mathrm{~cm}^{-1}$. Mass spectra were recorded on LCMsD mass spectrometer.

\section{Analytical data of $1 \mathrm{a}, 1 \mathrm{1}, \mathbf{1 j}$ and $1 \mathrm{~m}$}

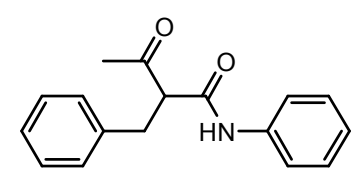

\section{2-Benzyl-3-oxo- $N$-phenylbutanamide (1a)}

White solid: mp 97-98 ${ }^{\circ} \mathrm{C} ;{ }^{1} \mathrm{H}$ NMR $\left(500 \mathrm{MHz}, \mathrm{CDCl}_{3}\right) \delta=2.14(\mathrm{~s}, 3 \mathrm{H}), 3.18-3.23(\mathrm{~m}, 1 \mathrm{H}), 3.29-3.33$ $(\mathrm{m}, 1 \mathrm{H}), 3.80(\mathrm{t}, J=7.5,1 \mathrm{H}), 7.11(\mathrm{t}, J=7.0,1 \mathrm{H}), 7.19(\mathrm{~d}, J=7.5,2 \mathrm{H}), 7.22(\mathrm{~d}, J=7.0,1 \mathrm{H}), 7.24-7.31$ $(\mathrm{m}, 4 \mathrm{H}), 7.45(\mathrm{~d}, J=8.0,2 \mathrm{H}), 8.30(\mathrm{~s}, 1 \mathrm{H}) ;{ }^{13} \mathrm{C} \mathrm{NMR}\left(125 \mathrm{MHz}, \mathrm{CDCl}_{3}\right) \delta=30.4,37.1,63.4,120.1$, 124.7, 127.0, 128.7, 128.8, 128.9, 137.3, 137.4, 166.2, 207.4; Anal. Calcd for $\mathrm{C}_{17} \mathrm{H}_{17} \mathrm{NO}_{2}: \mathrm{C}, 76.38 ; \mathrm{H}$, 6.41; N, 5.24. Found: C, 76.31; H, 6.51; N, 5.13.

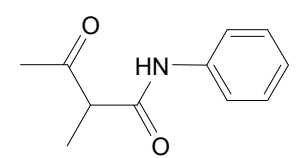

\section{2-Methyl-3-oxo- $N$-phenylbutanamide (1f)}

White solid: mp 120-122 ${ }^{\circ} \mathrm{C} ;{ }^{1} \mathrm{H}$ NMR $\left(500 \mathrm{MHz}, \mathrm{CDCl}_{3}\right) \delta=2.30(\mathrm{~s}, 3 \mathrm{H}), 3.53-3.57(\mathrm{~m}, 1 \mathrm{H}), 7.11(\mathrm{t}$, $J=7.0,1 \mathrm{H}), 7.31(\mathrm{t}, J=7.5,2 \mathrm{H}), 7.52(\mathrm{~d}, J=7.5,2 \mathrm{H}), 8.44(\mathrm{~s}, 1 \mathrm{H}) ;{ }^{13} \mathrm{C} \mathrm{NMR}\left(125 \mathrm{MHz}, \mathrm{CDCl}_{3}\right) \delta=$ 15.4, 29.1, 55.6, 120.2, 124.8, 129.2, 137.8, 167.8, 208.5; Anal. Calcd for $\mathrm{C}_{11} \mathrm{H}_{13} \mathrm{NO}_{2}$ : C, 69.09; $\mathrm{H}$, $6.85 ; \mathrm{N}, 7.32$. Found: C, 69.21; H, 6.97; N, 7.23.

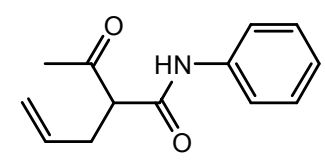

\section{2-Acetyl- $N$-phenylpent-4-enamide (1j)}

White solid: $\mathrm{mp} 77-78{ }^{\circ} \mathrm{C} ;{ }^{1} \mathrm{H}$ NMR $\left(500 \mathrm{MHz}, \mathrm{CDCl}_{3}\right) \delta=2.30(\mathrm{~s}, 3 \mathrm{H}), 2.71(\mathrm{t}, J=7.0,2 \mathrm{H}), 3.59$ (t, $J=7.0,1 \mathrm{H}), 5.09-5.16(\mathrm{~m}, 2 \mathrm{H}), 5.74-5.80(\mathrm{~m}, 1 \mathrm{H}), 7.12(\mathrm{t}, J=8.0,1 \mathrm{H}), 7.31(\mathrm{t}, J=8.0,2 \mathrm{H}), 7.51(\mathrm{~d}$, $J=8.0,2 \mathrm{H}), 8.39(\mathrm{~s}, 1 \mathrm{H}) ;{ }^{13} \mathrm{C} \mathrm{NMR}\left(125 \mathrm{MHz}, \mathrm{CDCl}_{3}\right) \delta=30.2,35.2,61.3,118.6,120.3,124.9$, 133.7, 137.6, 166.4, 207.8; Anal. Calcd for $\mathrm{C}_{13} \mathrm{H}_{15} \mathrm{NO}_{2}$ : C, 71.87; H, 6.96; N, 6.45. Found: C, 71.75; H, 6.99; N, 6.57 .

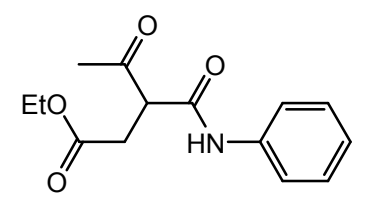


Ethyl 4-oxo-3-(phenylcarbamoyl)pentanoate (1 m)

White solid: $\mathrm{mp} 80-81{ }^{\circ} \mathrm{C} ;{ }^{1} \mathrm{H}$ NMR (500 MHz, $\left.\mathrm{CDCl}_{3}\right) \delta=1.22-1.28(\mathrm{~m}, 3 \mathrm{H}), 2.33$ (s, 2H), 2.91-2.96 (m, 1H), 3.04-3.10 (m, 1H), 3.87-3.89 (m, 1H), 4.13-4.20 (m, 2H), $7.11(\mathrm{t}, J=7.5,1 \mathrm{H}), 7.26-7.32(\mathrm{~m}$, 2H), 7.38-7.51 (m, 2H), $8.41(\mathrm{~s}, 1 \mathrm{H}) ;{ }^{13} \mathrm{C}$ NMR $\left(125 \mathrm{MHz}, \mathrm{CDCl}_{3}\right) \delta=14.1,28.9,33.2,57.5,61.4$, 119.9, 124.8, 129.0, 137.4, 166.1, 172.2, 203.9; Anal. Calcd for $\mathrm{C}_{14} \mathrm{H}_{17} \mathrm{NO}_{4}: \mathrm{C}, 63.87$; $\mathrm{H}, 6.51 ; \mathrm{N}, 5.32$; Found: C, 63.79; H, 6.60; N, 5.37.

\section{Synthesis and analytical data of 2a1-2o1, 2a2-2d2 and $2 \mathrm{f} 2$}

Typical procedure for the preparation of $\mathbf{2}$ (with $\mathbf{2 a 1}$ as an example): Vilsmeier reagent was prepared by adding $\mathrm{PBr}_{3}(5.0 \mathrm{mmol})$ dropwise to ice cold dry DMF $(5 \mathrm{~mL})$ under stirring. The mixture was then stirred for $10-15 \mathrm{~min}$ at $0{ }^{\circ} \mathrm{C}$. To the above Vilsmeier reagent was added 1a $(1.0 \mathrm{mmol})$ as a solution in DMF $(5.0 \mathrm{~mL})$. Then the mixture was allowed to warm to $80{ }^{\circ} \mathrm{C}$ and was stirred for $2.0 \mathrm{~h}$. After the staring material was consumed (monitored by TLC), the reaction mixture was poured into saturated sodium chloride aqueous $(50 \mathrm{~mL})$. The mixture was extracted with dichloromethane $(3 \times 20 \mathrm{~mL})$, the combined organic phase was washed with water $(3 \times 20 \mathrm{~mL})$, dried over MgSO4, filtered and concentrated in vacuo. The crude product was purified by flash chromatography (silica gel, petroleum ether: diethyl ether $=2: 1$ ) to give $\mathbf{2 a 1}$ as a white solid (256 mg, 71\%).<smiles>O=Cc1cn(-c2ccccc2)c(=O)c(Cc2ccccc2)c1Br</smiles>

\section{5-Benzyl-4-bromo-6-oxo-1-phenyl-1,6-dihydropyridine-3-carbaldehyde (2a1)}

White solid: mp 119-120 ${ }^{\circ} \mathrm{C} ;{ }^{1} \mathrm{H}$ NMR $\left(500 \mathrm{MHz}, \mathrm{CDCl}_{3}\right) \delta=4.21(\mathrm{~s}, 2 \mathrm{H}), 7.21(\mathrm{t}, J=7.0,1 \mathrm{H}), 7.28$ $(\mathrm{t}, J=7.0,2 \mathrm{H}), 7.34(\mathrm{~d}, J=7.0,2 \mathrm{H}), 7.46-7.52(\mathrm{~m}, 5 \mathrm{H}), 8.09(\mathrm{~s}, 1 \mathrm{H}), 10.11(\mathrm{~s}, 1 \mathrm{H}) ;{ }^{13} \mathrm{C}$ NMR $(125$ $\left.\mathrm{MHz}, \mathrm{CDCl}_{3}\right) \delta=36.2,115.5,126.2,126.6,128.4,129.0,129.4,129.6,133.1,135.2,137.9,139.4$, 141.2, 160.6, 188.7; IR (KBr) 1677, 1647, 1580, 1454, 1338, 1274, 1065, 749, $693 \mathrm{~cm}^{-1}$; Anal. Calcd for $\mathrm{C}_{19} \mathrm{H}_{14} \mathrm{BrNO}_{2}$ : C, 61.97; H, 3.83; N, 3.80; Found: C, 61.79; H, 3.95; N, 3.87. MS $m / z$ Calcd: 367.0; Found: $368.0\left[(\mathrm{M}+1)^{+}\right]$.

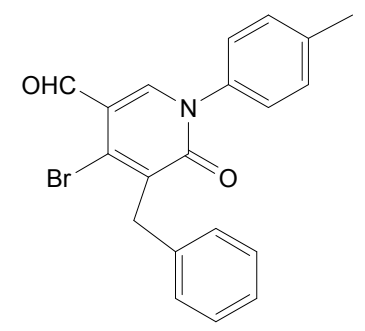

5-Benzyl-4-bromo-6-oxo-1-p-tolyl-1,6-dihydropyridine-3-carbaldehyde (2b1)

White solid: mp 158-159 ${ }^{\circ} \mathrm{C} ;{ }^{1} \mathrm{H}$ NMR $\left(500 \mathrm{MHz}, \mathrm{CDCl}_{3}\right) \delta=2.41$ (s, 3H), $4.20(\mathrm{~s}, 2 \mathrm{H}), 7.22$ (d, $J=$ 8.0, 3H), 7.26-7.31 (m, 4H), $7.46(\mathrm{~d}, J=8.0,2 \mathrm{H}), 8.08(\mathrm{~s}, 1 \mathrm{H}), 10.11(\mathrm{~s}, 1 \mathrm{H}) ;{ }^{13} \mathrm{C}$ NMR $(125 \mathrm{MHz}$, 
$\left.\mathrm{CDCl}_{3}\right) \delta=21.2,36.2,115.3,125.9,126.5,128.4,129.1,130.1,133.0,135.2,136.8,138.0,139.6$, 141.3, 160.7, 188.8; Anal. Calcd for $\mathrm{C}_{20} \mathrm{H}_{16} \mathrm{BrNO}_{2}$ : C, 62.84; H, 4.22; N, 3.66; Found: C, 62.83; H, $4.35 ; \mathrm{N}, 3.49$.<smiles>COc1ccc(-n2cc(C=O)c(Br)c(Cc3ccccc3)c2=O)cc1</smiles>

5-Benzyl-4-bromo-1-(4-methoxyphenyl)-6-oxo-1,6-dihydropyridine-3-carbaldehyde (2c1)

White solid: mp 102-103 ${ }^{\circ} \mathrm{C} ;{ }^{1} \mathrm{H}$ NMR $\left(500 \mathrm{MHz}, \mathrm{CDCl}_{3}\right) \delta=3.85$ (s, 3H), $4.20(\mathrm{~s}, 2 \mathrm{H}), 6.99(\mathrm{~d}, J=$ 9.0, 2H), 7.21-7.29 (m, 5H), $7.46(\mathrm{~d}, J=7.0,2 \mathrm{H}), 8.08(\mathrm{~s}, 1 \mathrm{H}), 10.11(\mathrm{~s}, 1 \mathrm{H}) ;{ }^{13} \mathrm{C}$ NMR $(125 \mathrm{MHz}$, $\left.\mathrm{CDCl}_{3}\right) \delta=36.5,55.9,114.9,115.6,126.8,127.6,128.6,129.3,132.4,133.2,135.4,138.3,141.6$, 160.3, 161.1, 189.1; Anal. Calcd for $\mathrm{C}_{20} \mathrm{H}_{16} \mathrm{BrNO}_{3}: \mathrm{C}, 60.32 ; \mathrm{H}, 4.05 ; \mathrm{N}, 3.52$; Found: $\mathrm{C}, 60.37$; , $4.13 ; \mathrm{N}, 3.63$.<smiles>O=Cc1cn(-c2ccc(Cl)cc2)c(=O)c(Cc2ccccc2)c1Br</smiles>

5-Benzyl-4-bromo-1-(4-chlorophenyl)-6-oxo-1,6-dihydropyridine-3-carbaldehyde (2d1)

White solid: $\mathrm{mp} 163-164{ }^{\circ} \mathrm{C} ;{ }^{1} \mathrm{H}$ NMR $\left(500 \mathrm{MHz}, \mathrm{CDCl}_{3}\right) \delta=4.20(\mathrm{~s}, 2 \mathrm{H}), 7.22-7.31(\mathrm{~m}, 5 \mathrm{H})$, 7.44-7.49 (m, 4H), $8.05(\mathrm{~s}, 1 \mathrm{H}), 10.12(\mathrm{~s}, 1 \mathrm{H}) ;{ }^{13} \mathrm{C} \mathrm{NMR}\left(125 \mathrm{MHz}, \mathrm{CDCl}_{3}\right) \delta=36.2,115.6,126.6$, 127.6, 128.4, 129.0, 129.7, 133.2, 135.3, 135.5, 137.7, 137.7, 140.6, 160.4, 188.7; Anal. Calcd for $\mathrm{C}_{19} \mathrm{H}_{13} \mathrm{BrClNO}_{2}$ : C, 56.67; H, 3.25; N, 3.48; Found: C, 56.77; H, 3.19; N, 3.55.<smiles>Cn1cc(C=O)c(Br)c(Cc2ccccc2)c1=O</smiles>

\section{5-Benzyl-4-bromo-1-methyl-6-oxo-1,6-dihydropyridine-3-carbaldehyde (2e1)}

White solid: mp 115-116 ${ }^{\circ} \mathrm{C} ;{ }^{1} \mathrm{H}$ NMR $\left(500 \mathrm{MHz}, \mathrm{CDCl}_{3}\right) \delta=3.60(\mathrm{~s}, 3 \mathrm{H}), 4.17$ (s, 2H), $7.21(\mathrm{~d}, J=$ $7.0,1 \mathrm{H}), 7.28(\mathrm{~d}, J=7.0,2 \mathrm{H}), 7.39(\mathrm{~d}, J=7.0,2 \mathrm{H}), 8.05(\mathrm{~s}, 1 \mathrm{H}), 10.06(\mathrm{~s}, 1 \mathrm{H}) ;{ }^{13} \mathrm{C}$ NMR $(125 \mathrm{MHz}$, $\left.\mathrm{CDCl}_{3}\right) \delta=36.0,38.6,115.2,126.5,128.4,128.8,131.9,135.2,137.9,141.4,161.2,188.8$; Anal. Calcd for $\mathrm{C}_{14} \mathrm{H}_{12} \mathrm{BrNO}_{2}$ : C, 54.92; H, 3.95; N, 4.58; Found: C, 54.87; H, 3.99; N, 4.43. 
<smiles>Cc1c(Br)c(C=O)cn(-c2ccccc2)c1=O</smiles>

\section{4-Bromo-5-methyl-6-oxo-1-phenyl-1,6-dihydropyridine-3-carbaldehyde (2f1)}

White solid: $\mathrm{mp} 129-130{ }^{\circ} \mathrm{C} ;{ }^{1} \mathrm{H}$ NMR $\left(500 \mathrm{MHz}, \mathrm{CDCl}_{3}\right) \delta=2.37(\mathrm{~s}, 3 \mathrm{H}), 7.34-7.35(\mathrm{~m}, 2 \mathrm{H})$, 7.48-7.52 (m, 3H), 8.05 (s, 1H), $10.09(\mathrm{~s}, 1 \mathrm{H}),{ }^{13} \mathrm{C} \mathrm{NMR}\left(125 \mathrm{MHz}, \mathrm{CDCl}_{3}\right) \delta=17.2,115.6,126.4$, 129.6, 129.7, 130.7, 134.6, 139.7, 140.6, 160.9, 189.0; IR (KBr) 1702, 1644, 1587, 1538, 1454, 1337 , 1267, 1085, 762, $689 \mathrm{~cm}^{-1}$; Anal. Calcd for $\mathrm{C}_{13} \mathrm{H}_{10} \mathrm{BrNO}_{2}$ : C, 53.45; H, 3.45; N, 4.79; Found: C, 53.54; H, 3.39; N, 4.87 .<smiles>Cc1ccc(-n2cc(C=O)c(Br)c(C)c2=O)cc1</smiles>

\section{4-Bromo-5-methyl-6-oxo-1-p-tolyl-1,6-dihydropyridine-3-carbaldehyde (2g1)}

White solid: mp 171-172 ${ }^{\circ} \mathrm{C} ;{ }^{1} \mathrm{H}$ NMR $\left(500 \mathrm{MHz}, \mathrm{CDCl}_{3}\right) \delta=2.37$ (s, 3H), 2.42 (s, 3H), $7.22(\mathrm{~d}, J=$ 8.0, 2H), $7.31(\mathrm{~d}, J=8.0,2 \mathrm{H}), 8.05(\mathrm{~s}, 1 \mathrm{H}), 10.10(\mathrm{~s}, 1 \mathrm{H}) ;{ }^{13} \mathrm{C} \mathrm{NMR}\left(125 \mathrm{MHz}, \mathrm{CDCl}_{3}\right) \delta=17.0,21.2$, 115.4, 126.0, 130.2, 130.5, 134.4, 137.1, 139.6, 140.7, 160.9, 188.9; Anal. Calcd for $\mathrm{C}_{14} \mathrm{H}_{12} \mathrm{BrNO}_{2}$ : C, 54.92; H, 3.95; N, 4.58; Found: C, 54.99; H, 3.87; N, 4.67.<smiles>COc1ccc(-n2cc(C=O)c(Br)c(C)c2=O)cc1</smiles>

\section{4-Bromo-1-(4-methoxyphenyl)-5-methyl-6-oxo-1,6-dihydropyridine-3-carbaldehyde (2h1)}

White solid: mp 151-152 ${ }^{\circ} \mathrm{C} ;{ }^{1} \mathrm{H}$ NMR $\left(500 \mathrm{MHz}, \mathrm{CDCl}_{3}\right) \delta=2.36$ (s, 3H), 3.86 (s, 3H), $7.00(\mathrm{~d}, J=$ $8.5,2 \mathrm{H}), 7.26(\mathrm{~d}, J=8.5,2 \mathrm{H}), 8.04(\mathrm{~s}, 1 \mathrm{H}), 10.09(\mathrm{~s}, 1 \mathrm{H}) ;{ }^{13} \mathrm{C} \mathrm{NMR}\left(125 \mathrm{MHz}, \mathrm{CDCl}_{3}\right) \delta=17.0,55.7$, 114.7, 115.3, 127.4, 130.4, 132.3, 134.4, 140.8, 160.1, 161.1, 188.9; Anal. Calcd for $\mathrm{C}_{14} \mathrm{H}_{12} \mathrm{BrNO}_{3}$ : C, 52.20; H, 3.75; N, 4.35; Found: C, 52.31; H, 3.79; N, 4.33 .<smiles>Cc1ccccc1-n1cc(C=O)c(Br)c(C)c1=O</smiles>

\section{4-Bromo-5-methyl-6-oxo-1-o-tolyl-1,6-dihydropyridine-3-carbaldehyde (2i1)}

White solid: mp 138-139 ${ }^{\circ} \mathrm{C} ;{ }^{1} \mathrm{H}$ NMR $\left(500 \mathrm{MHz}, \mathrm{CDCl}_{3}\right) \delta=2.13$ (s, 3H), 2.38 (s, 3H), $7.15(\mathrm{~d}, J=$ 7.5, 1H), 7.32-7.41 (m, 3H), $7.95(\mathrm{~s}, 1 \mathrm{H}), 10.11(\mathrm{~s}, 1 \mathrm{H}) ;{ }^{13} \mathrm{C} \mathrm{NMR}\left(125 \mathrm{MHz}, \mathrm{CDCl}_{3}\right) \delta=16.9,17.5$, 115.3, 126.7, 127.3, 129.9, 130.5, 131.3, 134.5, 134.6, 138.8, 140.6, 160.4, 188.8; Anal. Calcd for $\mathrm{C}_{14} \mathrm{H}_{12} \mathrm{BrNO}_{2}$ : C, 54.92; H, 3.95; N, 4.58; Found: C, 54.99; H, 3.87; N, 4.67. 
<smiles>C=CCc1c(Br)c(C=O)cn(-c2ccccc2)c1=O</smiles>

\section{5-Allyl-4-bromo-6-oxo-1-phenyl-1,6-dihydropyridine-3-carbaldehyde (2j1)}

White solid: $\mathrm{mp} 110-11{ }^{\circ} \mathrm{C} ;{ }^{1} \mathrm{H}$ NMR $\left(500 \mathrm{MHz}, \mathrm{CDCl}_{3}\right) \delta=3.61(\mathrm{~d}, J=6.5,2 \mathrm{H}), 5.12(\mathrm{~d}, J=10.5$, $1 \mathrm{H}), 5.25(\mathrm{~d}, J=16.0,1 \mathrm{H}), 5.89-5.94(\mathrm{~m}, 1 \mathrm{H}), 7.36(\mathrm{~d}, J=7.0,2 \mathrm{H}), 7.47-7.53(\mathrm{~m}, 3 \mathrm{H}), 8.09(\mathrm{~s}, 1 \mathrm{H})$, $10.11(\mathrm{~s}, 1 \mathrm{H}) ;{ }^{13} \mathrm{C} \mathrm{NMR}\left(125 \mathrm{MHz}, \mathrm{CDCl}_{3}\right) \delta=35.1,115.6,117.4,126.4,129.6,129.7,132.0,132.5$, 135.1, 139.6, 141.2, 160.4, 188.9; IR (KBr) 1695, 1644, 1581, 1534, 1454, 1340, 1267, 1064, 774, $691 \mathrm{~cm}^{-1}$; Anal. Calcd for $\mathrm{C}_{15} \mathrm{H}_{12} \mathrm{BrNO}_{2}: \mathrm{C}, 56.62 ; \mathrm{H}, 3.80 ; \mathrm{N}, 4.40$; Found: $\mathrm{C}, 56.77 ; \mathrm{H}, 3.87 ; \mathrm{N}$, 4.55 .<smiles>C=CCc1c(Br)c(C=O)cn(-c2ccc(C)cc2)c1=O</smiles>

\section{5-Allyl-4-bromo-6-oxo-1-p-tolyl-1,6-dihyd ropyridine-3-carbaldehyde (2k1)}

White solid: mp 119-120 ${ }^{\circ} \mathrm{C} ;{ }^{1} \mathrm{H}$ NMR $\left(500 \mathrm{MHz}, \mathrm{CDCl}_{3}\right) \delta=2.42(\mathrm{~s}, 3 \mathrm{H}), 3.60(\mathrm{~d}, J=1.5,2 \mathrm{H}), 5.11$ $(\mathrm{d}, J=9.5,1 \mathrm{H}), 5.25(\mathrm{~d}, J=17.5,1 \mathrm{H}), 5.88-5.93(\mathrm{~m}, 1 \mathrm{H}), 7.23(\mathrm{~d}, J=7.0,2 \mathrm{H}), 7.30(\mathrm{~d}, J=7.0,2 \mathrm{H})$, $8.08(\mathrm{~s}, 1 \mathrm{H}), 10.10(\mathrm{~s}, 1 \mathrm{H}) ;{ }^{13} \mathrm{C} \mathrm{NMR}\left(125 \mathrm{MHz}, \mathrm{CDCl}_{3}\right) \delta=21.2,34.9,115.3,117.2,125.9,130.1$, 131.6, 132.4, 134.8, 136.8, 139.6, 141.1, 160.3, 188.8; Anal. Calcd for $\mathrm{C}_{16} \mathrm{H}_{14} \mathrm{BrNO}_{2}$ : C, 57.85; $\mathrm{H}$, 4.25; N, 4.22; Found: C, 57.77; H, 4.33; N, 4.17 .<smiles>C=CCc1c(Br)c(C=O)cn(-c2ccc(OC)cc2)c1=O</smiles>

\section{5-Allyl-4-bromo-1-(4-methoxyphenyl)-6-oxo-1,6-dihydropyridine-3-carbaldehyde (211)}

White solid: $\mathrm{mp} 90-91{ }^{\circ} \mathrm{C} ;{ }^{1} \mathrm{H}$ NMR $\left(500 \mathrm{MHz}, \mathrm{CDCl}_{3}\right) \delta=3.54(\mathrm{~d}, J=6.5,2 \mathrm{H}), 3.85(\mathrm{~s}, 3 \mathrm{H}), 5.10(\mathrm{~d}$, $J=9.5,1 \mathrm{H}), 5.22(\mathrm{~d}, J=17.0,1 \mathrm{H}), 5.88-5.93(\mathrm{~m}, 1 \mathrm{H}), 7.00(\mathrm{~d}, J=8.0,2 \mathrm{H}), 7.27(\mathrm{~d}, J=8.0,2 \mathrm{H}), 8.11$ $(\mathrm{s}, 1 \mathrm{H}), 10.13(\mathrm{~s}, 1 \mathrm{H}) ;{ }^{13} \mathrm{C}$ NMR $\left(125 \mathrm{MHz}, \mathrm{CDCl}_{3}\right) \delta=32.4,55.9,114.8,114.9,117.3,127.6,129.3$, 132.4, 132.8, 141.7, 143.4, 160.3, 161.2, 187.0; Anal. Calcd for $\mathrm{C}_{16} \mathrm{H}_{14} \mathrm{BrNO}_{3}: \mathrm{C}, 55.19 ; \mathrm{H}, 4.05 ; \mathrm{N}$, 4.02; Found: C, 55.11; H, 4.17; N, 4.03 . 
<smiles>CCOC(=O)Cc1c(Br)c(C=O)cn(-c2ccccc2)c1=O</smiles>

Ethyl 2-(4-bromo-5-formyl-2-oxo-1-phenyl-1,2-dihydropyridin-3-yl)acetate (2m1)

White solid: $\mathrm{mp} 105-106{ }^{\circ} \mathrm{C}$; ${ }^{1} \mathrm{H}$ NMR $\left(500 \mathrm{MHz}, \mathrm{CDCl}_{3}\right) \delta=1.27-1.30(\mathrm{~m}, 3 \mathrm{H}), 3.91(\mathrm{~s}, 2 \mathrm{H})$, 4.18-4.22 (m, 2H), $7.37(\mathrm{~d}, J=8.0,2 \mathrm{H}), 7.51(\mathrm{t}, J=8.0,3 \mathrm{H}), 8.14(\mathrm{~s}, 1 \mathrm{H}), 10.10(\mathrm{~s}, 1 \mathrm{H}) ;{ }^{13} \mathrm{C} \mathrm{NMR}$ $\left(125 \mathrm{MHz}, \mathrm{CDCl}_{3}\right) \delta=14.2,36.3,61.3,115.4,126.1,127.6,129.5,129.6,136.6,139.2,141.9,160.3$, 169.3, 188.3; IR (KBr) 1741, 1694, 1648, 1594, 1541, 1456, 1333, 1270, 1085, 768, $692 \mathrm{~cm}^{-1}$; Anal. Calcd for $\mathrm{C}_{16} \mathrm{H}_{14} \mathrm{BrNO}_{4}$ : C, 52.77; H, 3.87; N, 3.85; Found: C, 52.86; H, 3.93; N, 3.74 .<smiles>CCOC(=O)Cc1c(Br)c(C=O)cn(-c2ccc(C)cc2)c1=O</smiles>

Ethyl 2-(4-bromo-5-formyl-2-oxo-1-p-tolyl-1,2-dihydropyridin-3-yl)acetate (2n1)

White solid: $\mathrm{mp} 120-121{ }^{\circ} \mathrm{C} ;{ }^{1} \mathrm{H}$ NMR $\left(500 \mathrm{MHz}, \mathrm{CDCl}_{3}\right) \delta=1.28(\mathrm{t}, J=7.0,3 \mathrm{H}), 2.42(\mathrm{~s}, 3 \mathrm{H}), 3.90$ (s, 2H), 4.17-4.22 (m, 2H), $7.24(\mathrm{~d}, J=8.5,2 \mathrm{H}), 7.30(\mathrm{~d}, J=8.52 \mathrm{H}), 8.13(\mathrm{~s}, 1 \mathrm{H}), 10.8(\mathrm{~s}, 1 \mathrm{H}) ;{ }^{13} \mathrm{C}$ NMR $\left(125 \mathrm{MHz}, \mathrm{CDCl}_{3}\right) \delta=14.1,21.2,36.3,61.3,109.7,115.2,125.8,127.4,130.1,136.6,139.7$, 142.0, 160.4, 169.3, 188.4; Anal. Calcd for $\mathrm{C}_{17} \mathrm{H}_{16} \mathrm{BrNO}_{4}$ : C, 53.99; H, 4.26; N, 3.70; Found: C, 53.84; $\mathrm{H}, 4.31 ; \mathrm{N}, 3.79$.<smiles>CCOC(=O)Cc1c(Br)c(C=O)cn(-c2ccc(OC)cc2)c1=O</smiles>

Ethyl 2-(4-bromo-5-formyl-1-(4-methoxyphenyl)-2-oxo-1,2-dihydropyridin-3-yl)acetate (201)

White solid: $\mathrm{mp} 105-106{ }^{\circ} \mathrm{C} ;{ }^{1} \mathrm{H}$ NMR $\left(500 \mathrm{MHz}, \mathrm{CDCl}_{3}\right) \delta=1.28(\mathrm{t}, J=6.5,3 \mathrm{H}), 3.85(\mathrm{~s}, 3 \mathrm{H}), 3.89$ $(\mathrm{s}, 2 \mathrm{H}), 4.20(\mathrm{t}, J=6.5,2 \mathrm{H}), 6.99(\mathrm{~d}, J=8.0,2 \mathrm{H}), 7.28(\mathrm{~d}, J=8.0,2 \mathrm{H}), 8.13(\mathrm{~s}, 1 \mathrm{H}), 10.08(\mathrm{~s}, 1 \mathrm{H})$; ${ }^{13} \mathrm{C} \mathrm{NMR}\left(125 \mathrm{MHz}, \mathrm{CDCl}_{3}\right) \delta=14.1,36.3,55.6,61.2,114.6,115.2,127.2,127.4,131.9,136.5$, 142.1, 160.1, 160.5, 169.3, 188.4; Anal. Calcd for $\mathrm{C}_{17} \mathrm{H}_{16} \mathrm{BrNO}_{5}$ : C, 51.79; H, 4.09; N, 3.55; Found: $\mathrm{C}$, $51.71 ; \mathrm{H}, 4.18 ; \mathrm{N}, 3.62$. 
<smiles>O=Cc1cn(-c2ccccc2)c(=O)c(Cc2ccccc2)c1Cl</smiles>

\section{5-Benzyl-4-chloro-6-oxo-1-phenyl-1,6-dihydropyridine-3-carbaldehyde (2a2)}

White solid: mp 102-103 ${ }^{\circ} \mathrm{C}$; ${ }^{1} \mathrm{H}$ NMR $\left(500 \mathrm{MHz}, \mathrm{CDCl}_{3}\right) \delta=4.15$ (s, 2H), 7.21 (t, $\left.J=7.5,1 \mathrm{H}\right)$, 7.26-7.29 (m, 2H), $7.34(\mathrm{~d}, J=6.5,2 \mathrm{H}), 7.44-7.53(\mathrm{~m}, 5 \mathrm{H}), 8.11(\mathrm{~s}, 1 \mathrm{H}), 10.14(\mathrm{~s}, 1 \mathrm{H}) ;{ }^{13} \mathrm{C}$ NMR $(125$ $\left.\mathrm{MHz}, \mathrm{CDCl}_{3}\right) \delta=33.8,115.0,126.5,126.8,128.7,129.3,129.7,129.8,130.9,138.3,139.6,141.5$, 143.6, 161.3, 186.9; IR (KBr) 1680, 1649, 1593, 1533, 1453, 1342, 1274, 749, $691 \mathrm{~cm}^{-1}$; Anal. Calcd for $\mathrm{C}_{19} \mathrm{H}_{14} \mathrm{ClNO}_{2}$ : C, 70.48; H, 4.36; N, 4.33; Found: C, 70.33; H, 4.43; N, 4.39. MS m/z Calcd: 323.1; Found: $324.1\left[(\mathrm{M}+1)^{+}\right]$.<smiles>Cc1ccc(-n2cc(C=O)c(Cl)c(Cc3ccccc3)c2=O)cc1</smiles>

\section{5-Benzyl-4-chloro-6-oxo-1-p-tolyl-1,6-dihydropyridine-3-carbaldehyde (2b2)}

White solid: $\mathrm{mp} 81-82{ }^{\circ} \mathrm{C} ;{ }^{1} \mathrm{H}$ NMR $\left(500 \mathrm{MHz}, \mathrm{CDCl}_{3}\right) \delta=2.41(\mathrm{~s}, 3 \mathrm{H}), 4.15(\mathrm{~s}, 2 \mathrm{H}), 7.22(\mathrm{~d}, J=8.0$, $3 \mathrm{H}), 7.26-7.31(\mathrm{~m}, 4 \mathrm{H}), 7.45(\mathrm{~d}, J=7.0,2 \mathrm{H}), 8.10(\mathrm{~s}, 1 \mathrm{H}), 10.13(\mathrm{~s}, 1 \mathrm{H}) ;{ }^{13} \mathrm{C}$ NMR $\left(125 \mathrm{MHz}, \mathrm{CDCl}_{3}\right)$ $\delta=21.4,33.8,114.9,126.2,126.8,128.7,129.4,130.3,130.8,137.1,138.4,139.9,141.7,143.5$, 161.4, 186.9; Anal. Calcd for $\mathrm{C}_{20} \mathrm{H}_{16} \mathrm{ClNO}_{2}$ : C, 71.11; H, 4.77; N, 4.15; Found: C, 71.23; H, 4.72; N, 4.22 .<smiles>COc1ccc(-n2cc(C=O)c(Cl)c(Cc3ccccc3)c2=O)cc1</smiles>

\section{5-Benzyl-4-chloro-1-(4-methoxyphenyl)-6-oxo-1,6-dihydropyridine-3-carbaldehyde (2c2)}

White solid: mp 119-120 ${ }^{\circ} \mathrm{C} ;{ }^{1} \mathrm{H}$ NMR $\left(500 \mathrm{MHz}, \mathrm{CDCl}_{3}\right) \delta=3.84$ (s, 3H), 4.14 (s, 2H), 6.98 (t, $J=7.0$, 2H), 7.20-7.26 (m, 5H), 7.44 (d, $J=7.0,2 \mathrm{H}), 8.10(\mathrm{~s}, 1 \mathrm{H}), 10.13(\mathrm{~s}, 1 \mathrm{H}) ;{ }^{13} \mathrm{C} \mathrm{NMR}\left(125 \mathrm{MHz}, \mathrm{CDCl}_{3}\right)$ $\delta=33.5,55.6,114.5,114.6,126.5,127.3,128.4,129.1,130.4,132.1,138.1,141.5,143.2,160.0$, 161.3, 186.6; Anal. Calcd for $\mathrm{C}_{20} \mathrm{H}_{16} \mathrm{ClNO}_{3}$ : C, 67.90; H, 4.56; N, 3.96; Found: C, 67.83; H, 4.51; N, 3.99 . 
<smiles>O=Cc1cn(-c2ccc(Cl)cc2)c(=O)c(Cc2ccccc2)c1Cl</smiles>

\section{5-Benzyl-4-chloro-1-(4-chlorophenyl)-6-oxo-1,6-dihydropyridine-3-carbaldehyde (2d2)}

White solid: $\mathrm{mp} 147-149{ }^{\circ} \mathrm{C} ;{ }^{1} \mathrm{H}$ NMR $\left(500 \mathrm{MHz}, \mathrm{CDCl}_{3}\right) \delta=4.15(\mathrm{~s}, 2 \mathrm{H}), 7.21-7.23(\mathrm{~m}, 1 \mathrm{H})$, 7.27-7.31 (m, 4H), 7.43 (d, $J=7.5,2 \mathrm{H}), 7.49$ (d, $J=8.5,2 \mathrm{H}), 8.07(\mathrm{~s}, 1 \mathrm{H}), 10.15(\mathrm{~s}, 1 \mathrm{H}) ;{ }^{13} \mathrm{C} \mathrm{NMR}$ $\left(125 \mathrm{MHz}, \mathrm{CDCl}_{3}\right) \delta=33.8,115.2,126.9,127.9,128.7,129.3,130.0,131.0,135.8,138.0,138.2$, 141.0, 143.7, 161.1, 186.8; Anal. Calcd for $\mathrm{C}_{19} \mathrm{H}_{13} \mathrm{Cl}_{2} \mathrm{NO}_{2}$ : C, 63.71; H, 3.66; N, 3.91; Found: C, $63.88 ; \mathrm{H}, 3.60 ; \mathrm{N}, 3.78$.<smiles>Cc1c(Cl)c(C=O)cn(-c2ccccc2)c1=O</smiles>

\section{4-Chloro-5-methyl-6-oxo-1-phenyl-1,6-dihydropyridine-3-carbaldehyde (2f2)}

White solid: $\mathrm{mp} 130-131{ }^{\circ} \mathrm{C} ;{ }^{1} \mathrm{H}$ NMR $\left(500 \mathrm{MHz}, \mathrm{CDCl}_{3}\right) \delta=2.32(\mathrm{~s}, 3 \mathrm{H}), 7.34(\mathrm{~d}, J=7.0,2 \mathrm{H})$, 7.48-7.53 (m, 3H), $8.09(\mathrm{~s}, 1 \mathrm{H}), 10.13(\mathrm{~s}, 1 \mathrm{H}) ;{ }^{13} \mathrm{C} \mathrm{NMR}\left(125 \mathrm{MHz}, \mathrm{CDCl}_{3}\right) \delta=14.1,114.9,126.5$, 128.1, 129.7, 129.8, 139.8, 140.8, 143.0, 161.5, 187.0; Anal. Calcd for $\mathrm{C}_{13} \mathrm{H}_{10} \mathrm{ClNO}_{2}: \mathrm{C}, 63.04 ; \mathrm{H}$, 4.07; N, 5.66; Found: C, 63.13; H, 4.15; N, 5.53.

\section{Analytical data of $2 \mathrm{p} 2,3 \mathrm{p} 2,2 \mathrm{q} 2,3 \mathrm{q} 2$ and $4 \mathrm{a}$}<smiles>O=Cc1cn(-c2ccccc2)c(=O)cc1Cl</smiles>

\section{4-Chloro-6-oxo-1-phenyl-1,6-dihydropyridine-3-carbaldehyde (2p2)}

White solid: $\mathrm{mp} 149-151{ }^{\circ} \mathrm{C} ;{ }^{1} \mathrm{H}$ NMR $\left(500 \mathrm{MHz}, \mathrm{CDCl}_{3}\right) \delta=6.73(\mathrm{~s}, 1 \mathrm{H}), 7.35(\mathrm{~d}, J=7.0,2 \mathrm{H})$, 7.48-7.55 (m, 3H), 8.19 (s, 1H), $10.08(\mathrm{~s}, 1 \mathrm{H}) ;{ }^{13} \mathrm{C} \mathrm{NMR}\left(125 \mathrm{MHz}, \mathrm{CDCl}_{3}\right) \delta=114.9,120.1,126.3$, 129.8, 129.9, 139.1, 144.1, 146.6, 160.6, 185.9; Anal. Calcd for $\mathrm{C}_{12} \mathrm{H}_{8} \mathrm{ClNO}_{2}: \mathrm{C}, 61.69 ; \mathrm{H}, 3.45 ; \mathrm{N}$, 5.99; Found: C, 61.74; H, 3.52; N, 5.86. MS m/z Calcd: 233.6; Found: $233.6\left[\mathrm{M}^{+}\right]$.

Crystal data for 2p2: $\mathrm{C}_{12} \mathrm{H}_{8} \mathrm{ClNO}_{2}$, white crystal, $M=233.65$, Monoclinic, $\mathrm{P} 21 / \mathrm{c}, a=11.067(3) \AA, b=$ 12.890(4) $\AA, c=7.536(2) \AA, \alpha=90.00^{\circ}, \beta=103.019(4)^{\circ}, \gamma=90.00^{\circ}, V=1047.4(9) \AA 3, Z=4, T=$ 293(2), $F 000=480, R 1=0.0323, w R 2=0.0891 . C C D C$ deposition number: 667624. These data can be obtained free of charge via www.ccdc.cam.ac.uk/conts/retrieving.html (or from the Cambridge Crystallographic Data Center, 12 Union Road, Cambridge CB21EZ, UK; fax: (+44)1223-336-033; or deposit@ccdc.cam.ac.uk). 
<smiles>O=Cc1c(Cl)ccn(-c2ccccc2)c1=O</smiles>

\section{4-Chloro-2-oxo-1-phenyl-1,2-dihydropyridine-3-carbaldehyde (3p2)}

Yellow solid: $\mathrm{mp} 171-173{ }^{\circ} \mathrm{C} ;{ }^{1} \mathrm{H}$ NMR $\left(500 \mathrm{MHz}, \mathrm{CDCl}_{3}\right) \delta=6.41(\mathrm{~d}, J=7.0,1 \mathrm{H}), 7.37(\mathrm{~d}, J=7.5$, 2H), 7.49-7.55 (m, 4H), 10.39 (s, 1H); ${ }^{13} \mathrm{C}$ NMR (125 MHz, $\left.\mathrm{CDCl}_{3}\right) \delta=109.6,122.0,126.2,129.4$, 129.6, 139.1, 141.7, 150.5, 161.5, 188.5; Anal. Calcd for $\mathrm{C}_{12} \mathrm{H}_{8} \mathrm{ClNO}_{2}$ : C, 61.69; H, 3.45; N, 5.99; Found: C, 61.77; H, 3.51; N, 6.04. MS m/z Calcd: 233.6; Found: $233.6\left[\mathrm{M}^{+}\right]$.<smiles>Cc1ccc(-n2cc(C=O)c(Cl)cc2=O)cc1</smiles>

\section{4-Chloro-6-oxo-1-p-tolyl-1,6-dihydropyridine-3-carbaldehyde (2q2)}

White solid: mp 172-174 ${ }^{\circ} \mathrm{C} ;{ }^{1} \mathrm{H}$ NMR $\left(500 \mathrm{MHz}, \mathrm{CDCl}_{3}\right) \delta=2.42(\mathrm{~s}, 3 \mathrm{H}), 6.71(\mathrm{~s}, 1 \mathrm{H}), 7.22(\mathrm{~d}, J=$ $8.0,2 \mathrm{H}), 7.31(\mathrm{~d}, J=8.0,2 \mathrm{H}), 8.18(\mathrm{~s}, 1 \mathrm{H}), 10.07(\mathrm{~s}, 1 \mathrm{H}) ;{ }^{13} \mathrm{C} \mathrm{NMR}\left(125 \mathrm{MHz}, \mathrm{CDCl}_{3}\right) \delta=21.2$, 114.6, 119.7, 125.8, 130.2, 136.3, 139.8, 144.0, 146.3, 160.6, 185.8; Anal. Calcd for $\mathrm{C}_{13} \mathrm{H}_{10} \mathrm{ClNO}_{2}$ : $\mathrm{C}$, 63.04; H, 4.07; N, 5.66; Found: C, 63.11; H, 4.16; N, 5.61.<smiles>Cc1ccc(-n2ccc(Cl)c(C=O)c2=O)cc1</smiles>

\section{4-Chloro-2-oxo-1-p-tolyl-1,2-dihydropyridine-3-carbaldehyde (3q2)}

Yellow solid: mp 147-148 ${ }^{\circ} \mathrm{C}$; ${ }^{1} \mathrm{H}$ NMR $\left(500 \mathrm{MHz}, \mathrm{CDCl}_{3}\right) \delta=2.42(\mathrm{~s}, 3 \mathrm{H}), 6.38(\mathrm{~d}, J=7.0,1 \mathrm{H})$, $7.23(\mathrm{~d}, J=7.5,2 \mathrm{H}), 7.31-7.35(\mathrm{~m}, 2 \mathrm{H}), 7.50(\mathrm{~d}, J=7.0,1 \mathrm{H}), 10.38(\mathrm{~s}, 1 \mathrm{H}) ;{ }^{13} \mathrm{C}$ NMR $(125 \mathrm{MHz}$, $\left.\mathrm{CDCl}_{3}\right) \delta=21.4,109.7,122.1,126.1,130.4,136.8,139.8,142.1,150.6,161.8,188.8$; Anal. Calcd for $\mathrm{C}_{13} \mathrm{H}_{10} \mathrm{ClNO}_{2}: \mathrm{C}, 63.04 ; \mathrm{H}, 4.07$; N, 5.66; Found: $\mathrm{C}, 63.17 ; \mathrm{H}, 4.23 ; \mathrm{N}, 5.73$.<smiles>CC(Cl)=C(Cc1ccccc1)C(=O)Nc1ccccc1</smiles>

\section{2-Benzyl-3-chloro- $N$-phenylbut-2-enamide (4a)}

White solid: mp 111-112 ${ }^{\circ} \mathrm{C} ;{ }^{1} \mathrm{H}$ NMR (500 MHz, $\left.\mathrm{CDCl}_{3}\right) \delta=2.38$ (s, 3H), $3.90(\mathrm{~s}, 2 \mathrm{H}), 6.85(\mathrm{~s}, 1 \mathrm{H})$, $7.10(\mathrm{~s}, 1 \mathrm{H}), 7.27-7.28(\mathrm{~m}, 5 \mathrm{H}), 7.31-7.41(\mathrm{~m}, 4 \mathrm{H}) ;{ }^{13} \mathrm{C} \mathrm{NMR}\left(125 \mathrm{MHz}, \mathrm{CDCl}_{3}\right) \delta=24.7(1 \mathrm{C}), 38.1$ (1C), 120.2 (2C), 125.0 (1C), 127.3 (1C), 128.9 (2C), 129.3 (4C), 133.8 (1C), 135.8 (1C), 137.3 (1C),138.1 (1C), 166.3 (1C); Anal. Calcd for $\mathrm{C}_{17} \mathrm{H}_{16} \mathrm{ClNO}$ : C, 71.45; H, 5.64; N, 4.90; Found: $\mathrm{C}$, $71.53 ; \mathrm{H}, 5.71 ; \mathrm{N}, 7.76$. 
IV. Copies of NMR spectra for compounds 1- 4

$1 \mathbf{a}$

STANDARD PROTON PARAMETE 8

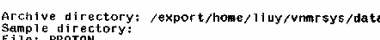

Pulse sequence: 52 ou

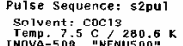

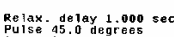

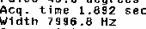

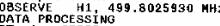

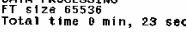
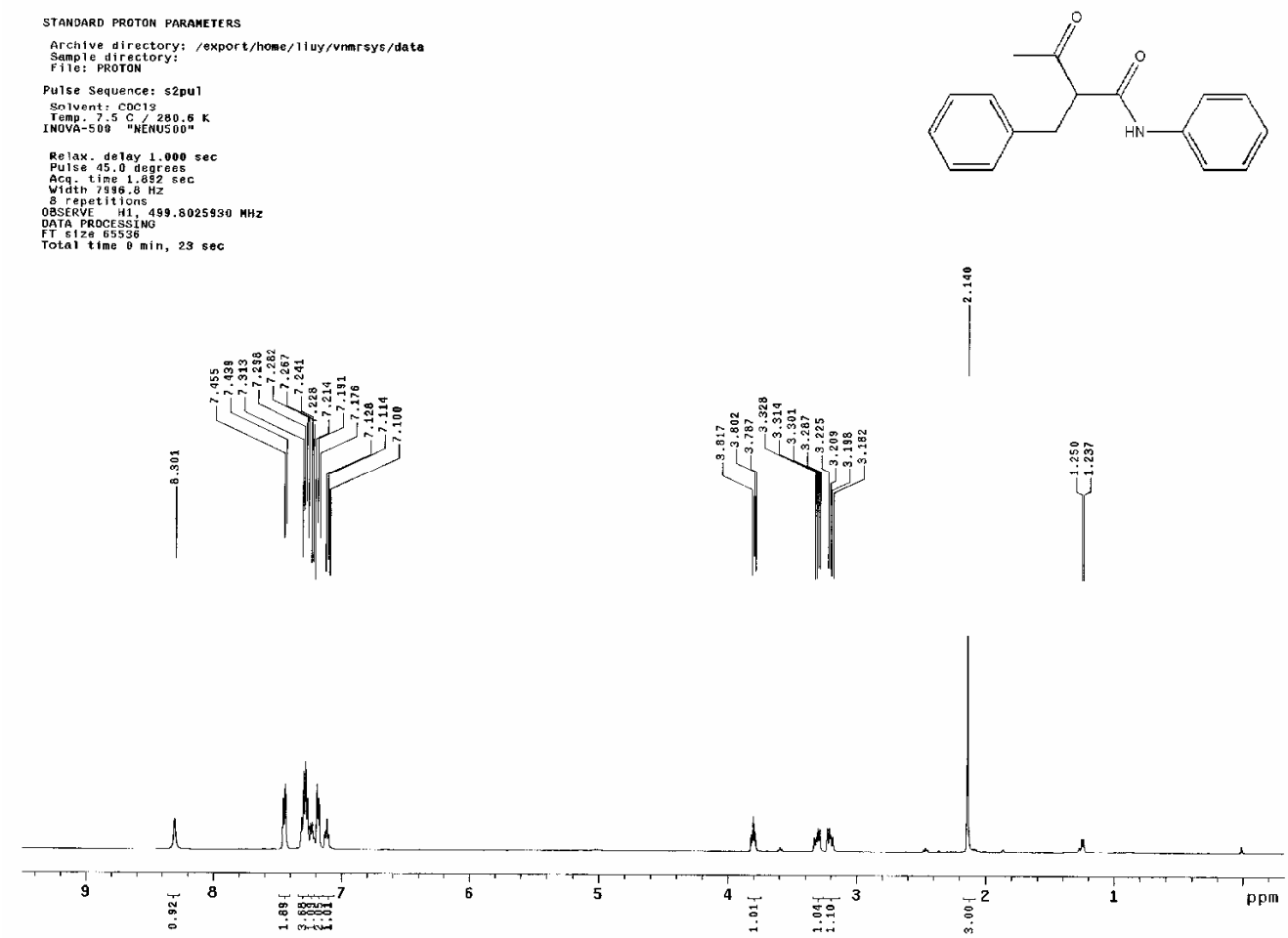

STANDARO CARBON PARAMETERS

Archlve directory: /export/home/1 iuy/unnrsys/data

Pulse Sequence: s2p

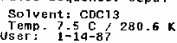

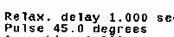

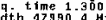

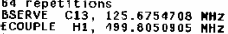

Power 10 de

off during de lay

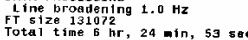
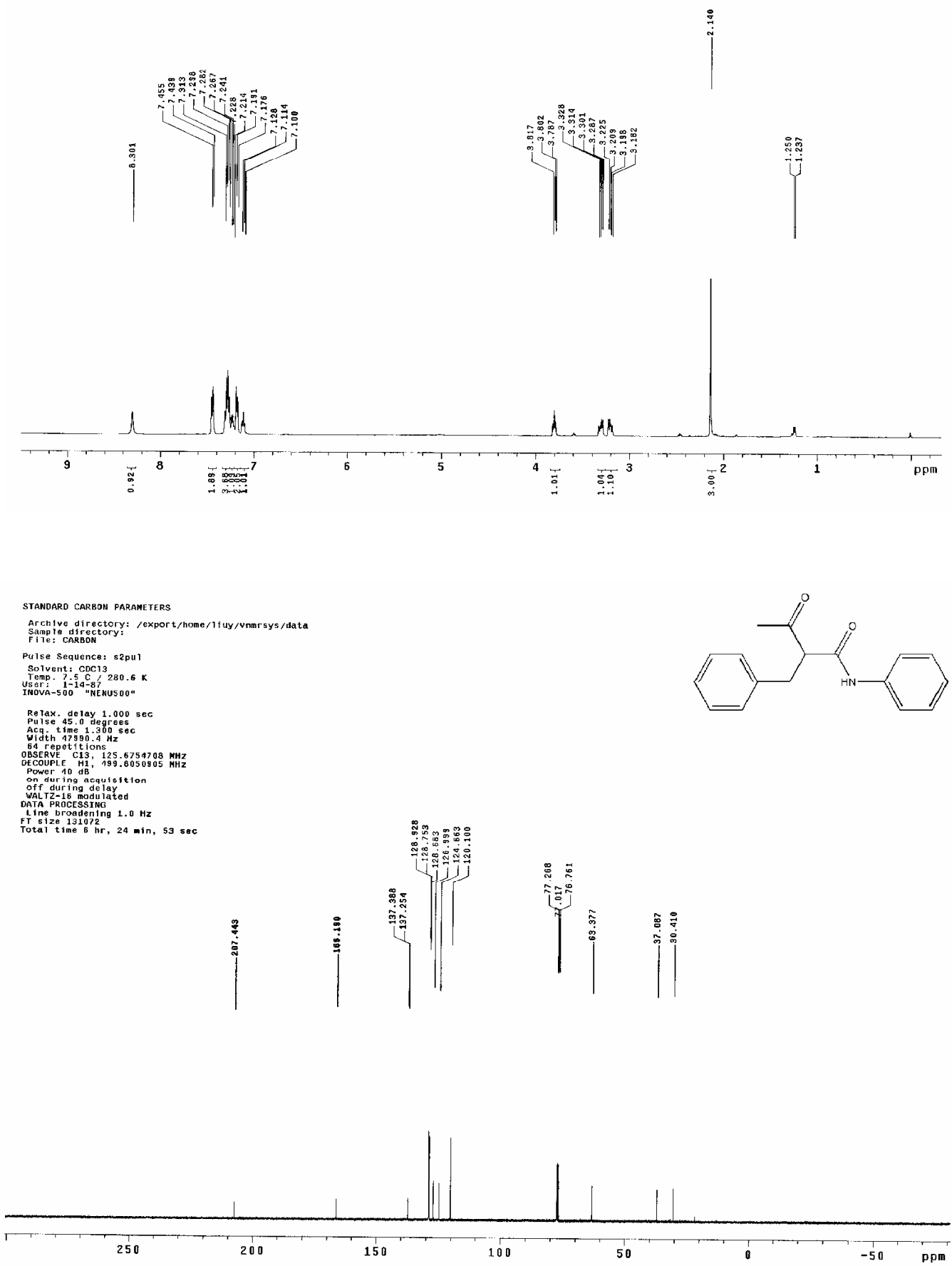
STANDARD PROTON PARAMETERS

Archive directory: /export/home/1 luy/vanr sys/data
Sample directory:

Pulse Sequence: sapul

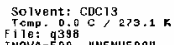

Relax. deg lay 1.000 sec

Pulse 45.0 degres

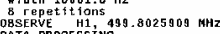

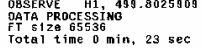

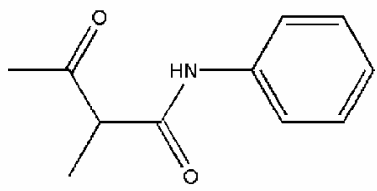

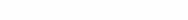
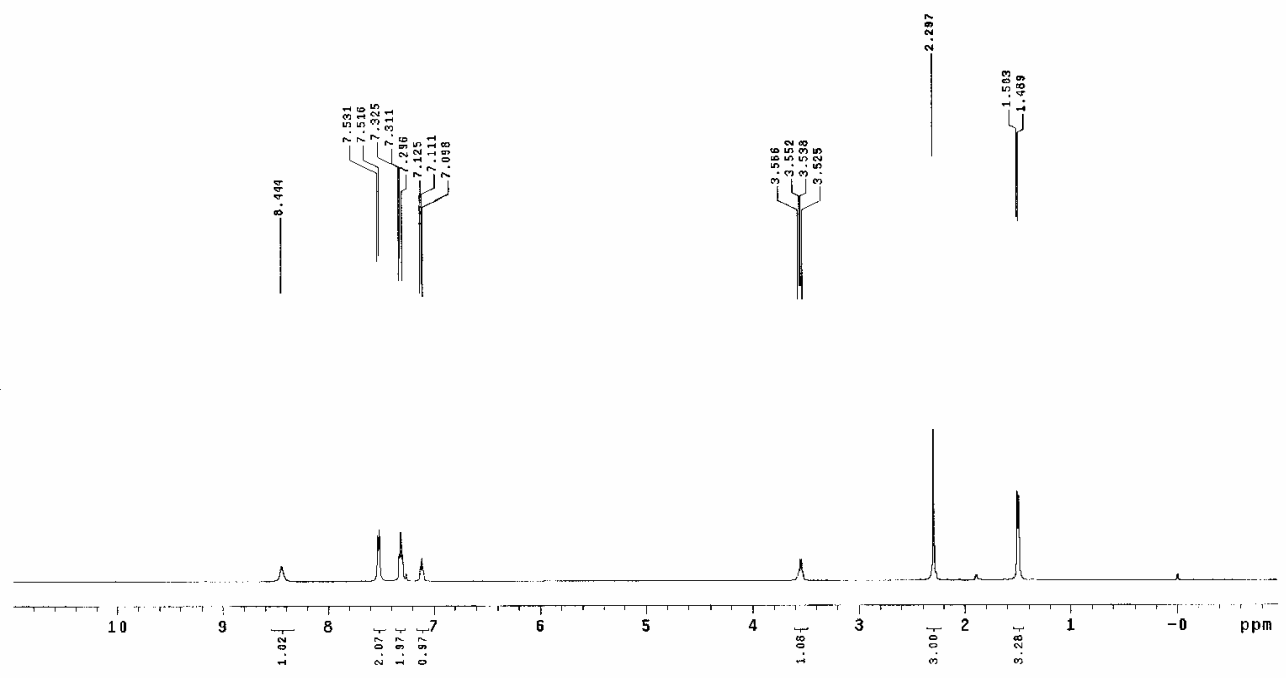

STANDARE CARBon PARaMETERS

Archive directory: export/home/11 uy/vnmrsys/data
Sample directory:
Fillo:

Pulse sequence: s2pur

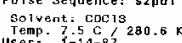

USer:
INOVA-500

Relax. delay $1.000 \mathrm{sec}$
pulse 45.0 degress
Alse

Ach. time 1.300 sec

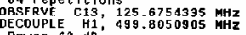

Fower $40 \mathrm{~dB}$
ont during acquisition
off

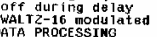

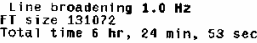
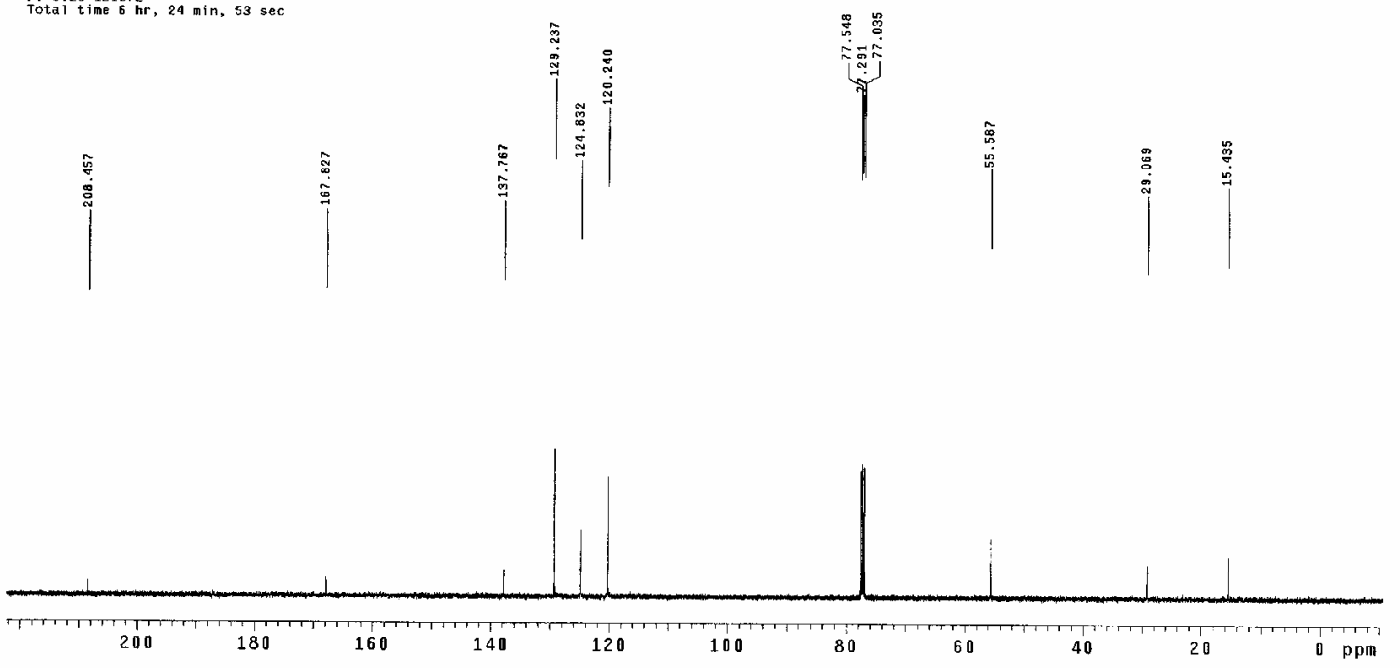
StANDARD PROTON PARAmETers

Archive directory: /export/home/1luy/unnrsys/late
Sample directory:

Pulse Sequence: s20u1

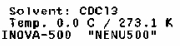

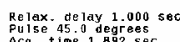

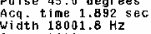

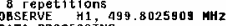

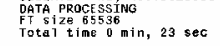
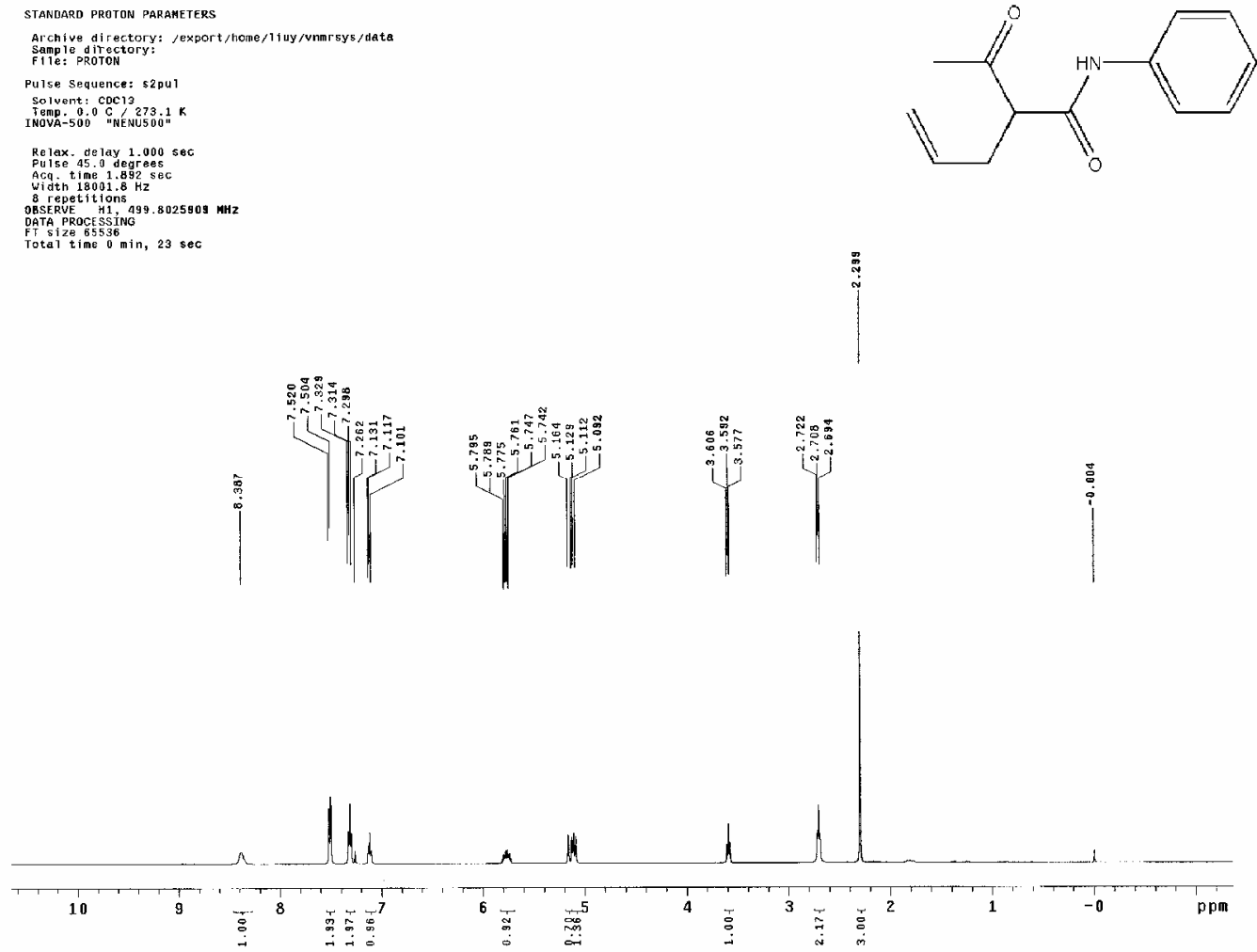

STANDARD CARBON PARAMETERS

Archive directory: /export/home/1iuy/umrsys/data
Saapole directory:

Pulso Soquences: s2pul

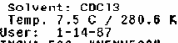

Relax. delay 1.000 sec
Pulse 45.0 degreas
pis

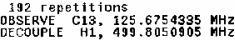

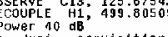

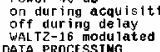

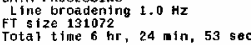

min, 53 sec

$\stackrel{m}{?}$

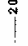

iٓj
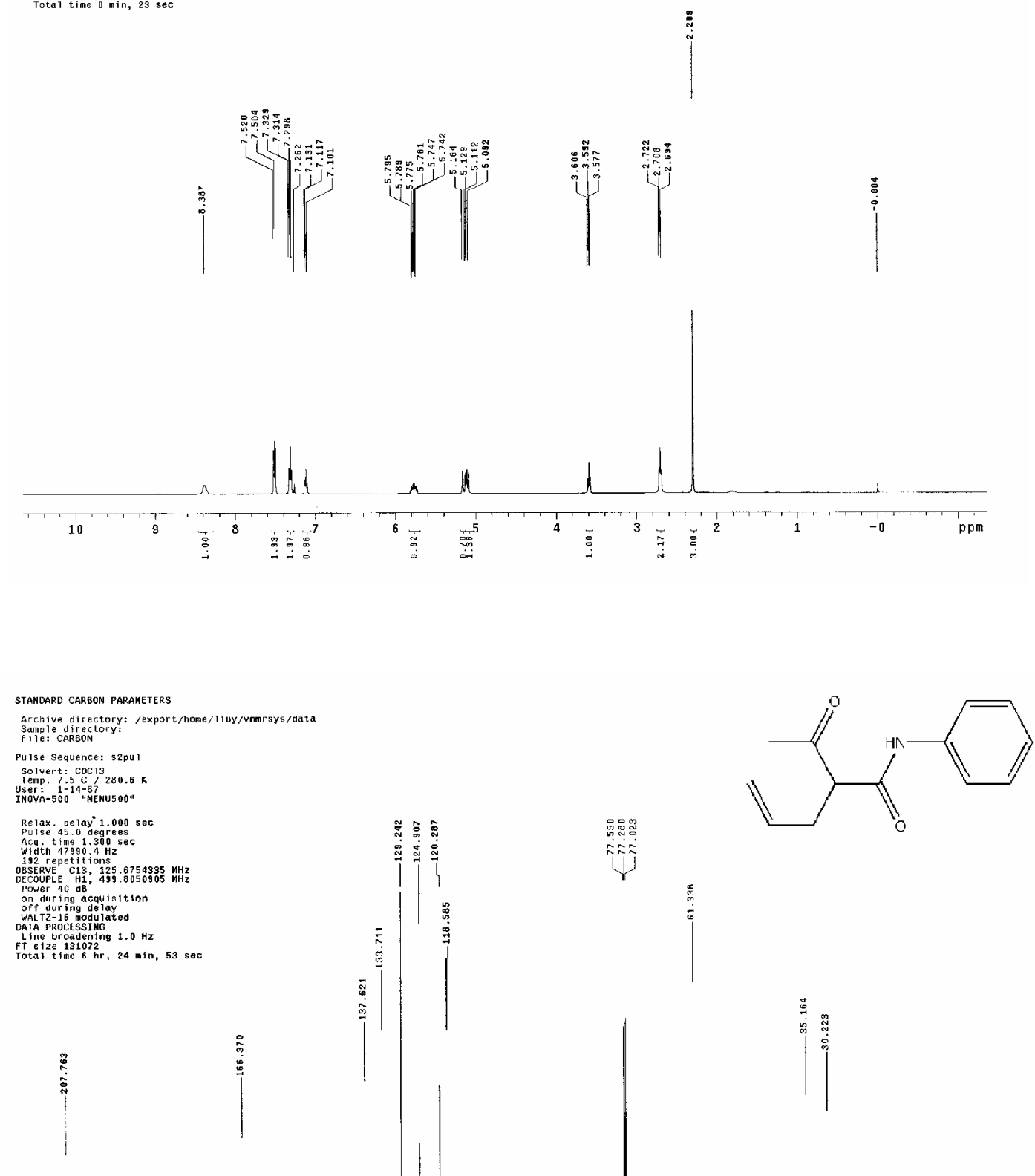

Tा1 200

$200 \quad 18$

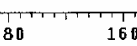

160 140

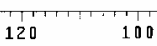

$80 \quad 60$

40 Tाग 20

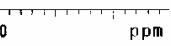


STANDARD PROTOU PARameters

Archive directory: /export/home/liuy/unmersys/data
Scmple directory:

Pulse Sequence: s2put

Solvent: $\mathrm{CDC}^{3}$
Temp. $0 \mathrm{C}, \mathrm{C}, 273.1 \mathrm{~K}$

Relax. delay $1.000 \mathrm{sec}$

Acq time $1.8925 \mathrm{sec}$

BeEpetitions
OBSERE

DAFA PROCESSING
FT SiZ 65536 .
Total time 0 uin, 23 sac
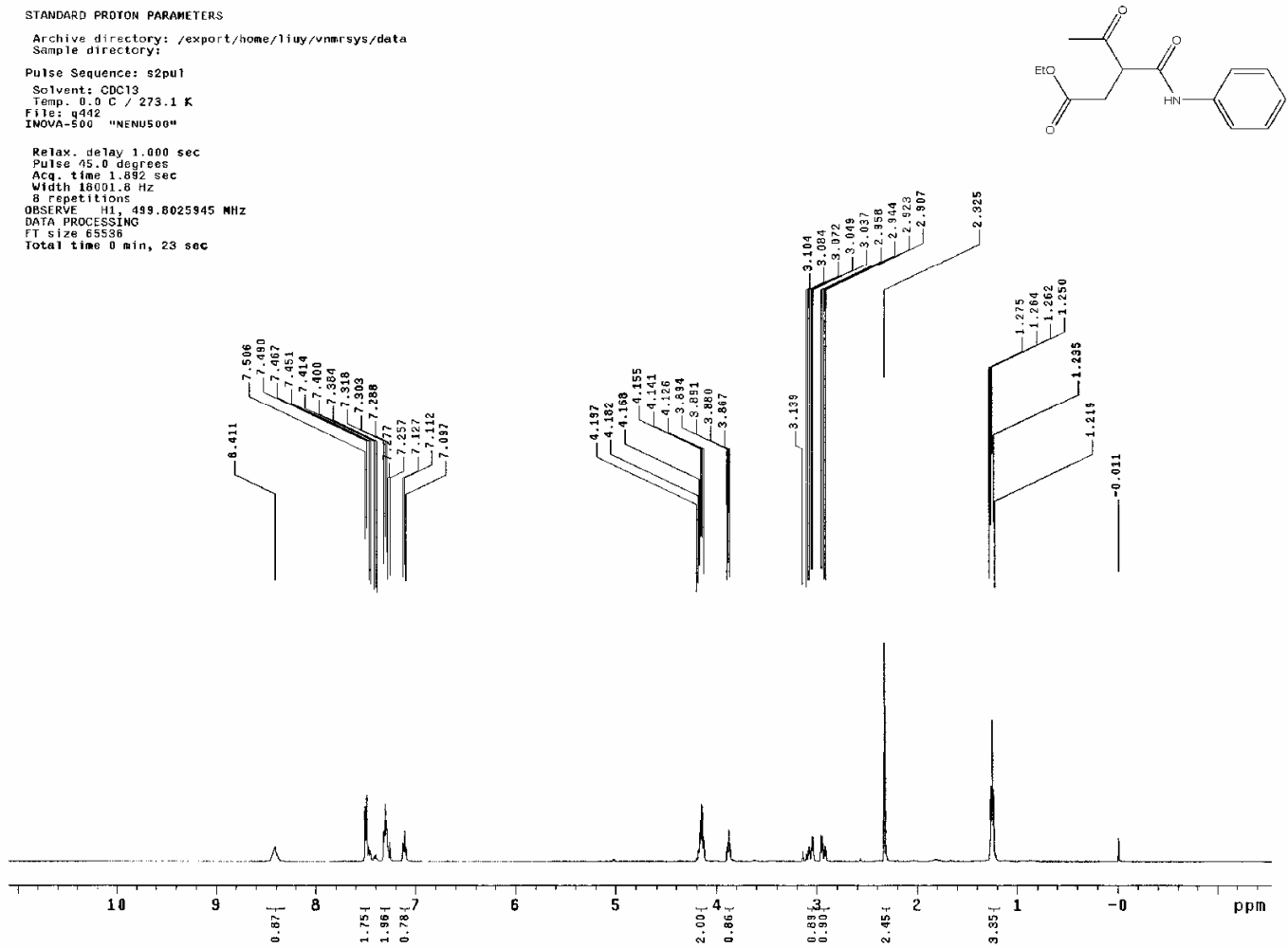

STANDARD CARBom PARAMETERS

Archive di rectory: /export/home/1 iuy/unnm sys/data

Pulse Sequence: $52 \mathrm{pur}$

Solvent: cocis $22 \mathrm{por}$

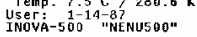

Relax de lay $1.000 \mathrm{sec}$
Pulse 45.0 degrees

Acq $t$ ine 1.300 sec

128 repetitions
OBSEVER C13, 125.6754628 MHZ
DECOUPLE H1, 499.8050905 KHHZ

oner 40 ab acquisition

uff dul irig delay
Wal Tz-16 modulated

tine urogaden1119 $1.0 \mathrm{~Hz}$

Total time $3 \mathrm{hr}, 12 \mathrm{~min}, 26 \mathrm{sec}$
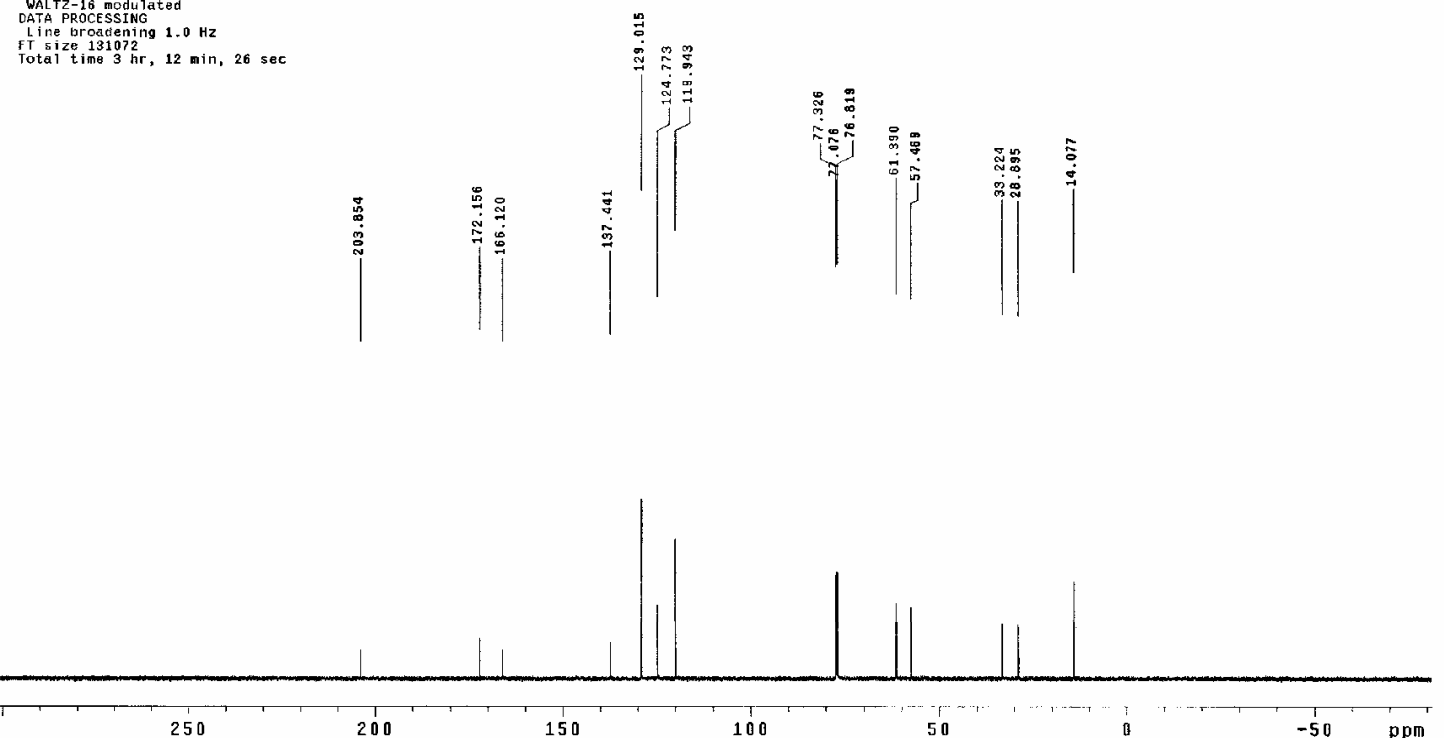
TANDARD PROTON PARAMETERS

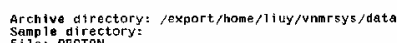

Pulse Sequence: s2pu

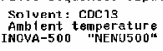

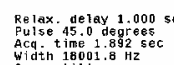

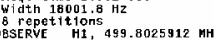

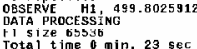
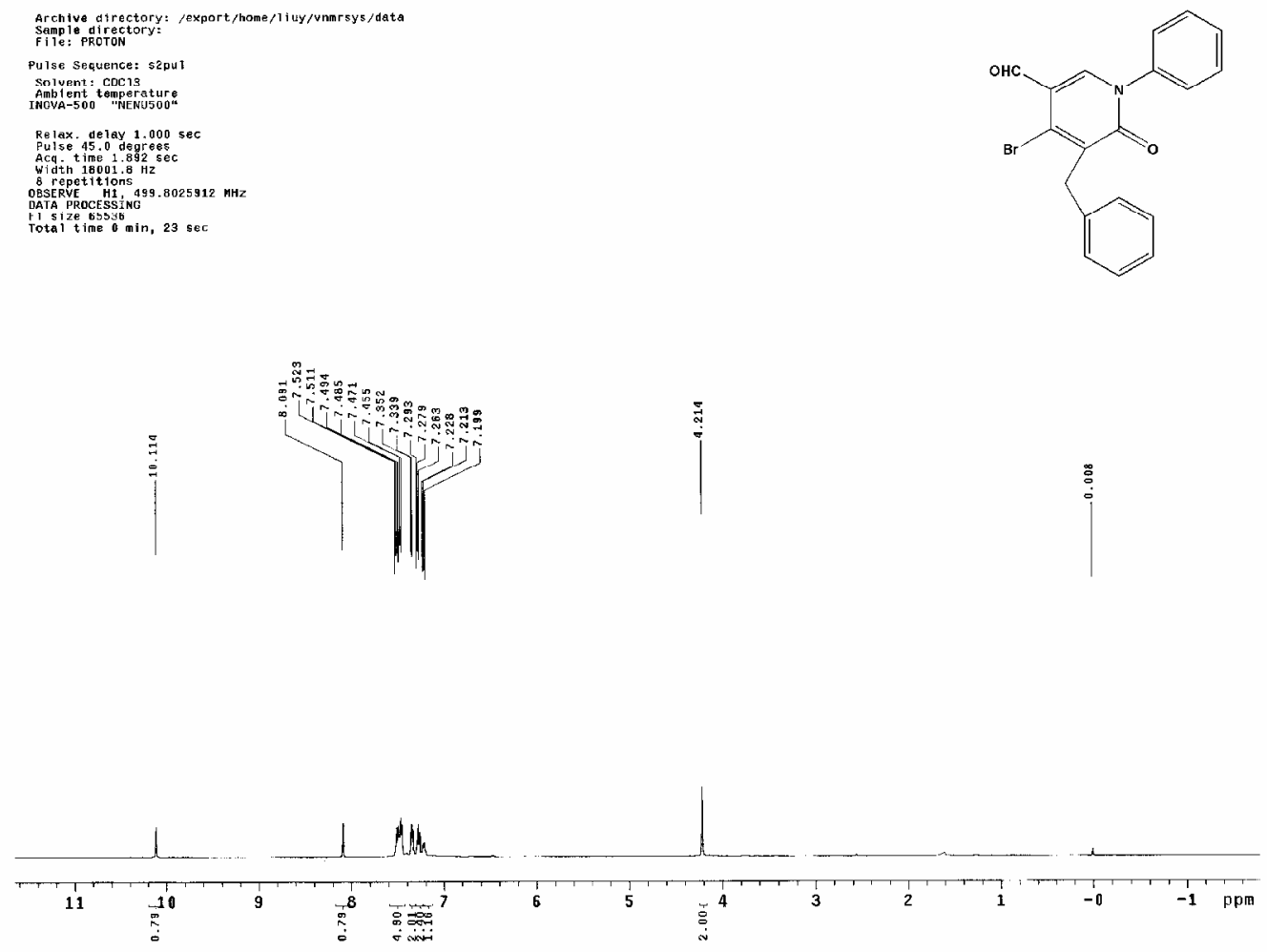

standaro carbon parameters

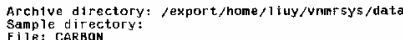

Pulse sequence: s2put

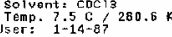

Pelax. delay 1.000

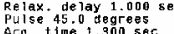

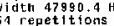

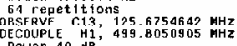

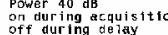

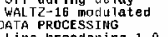

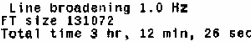
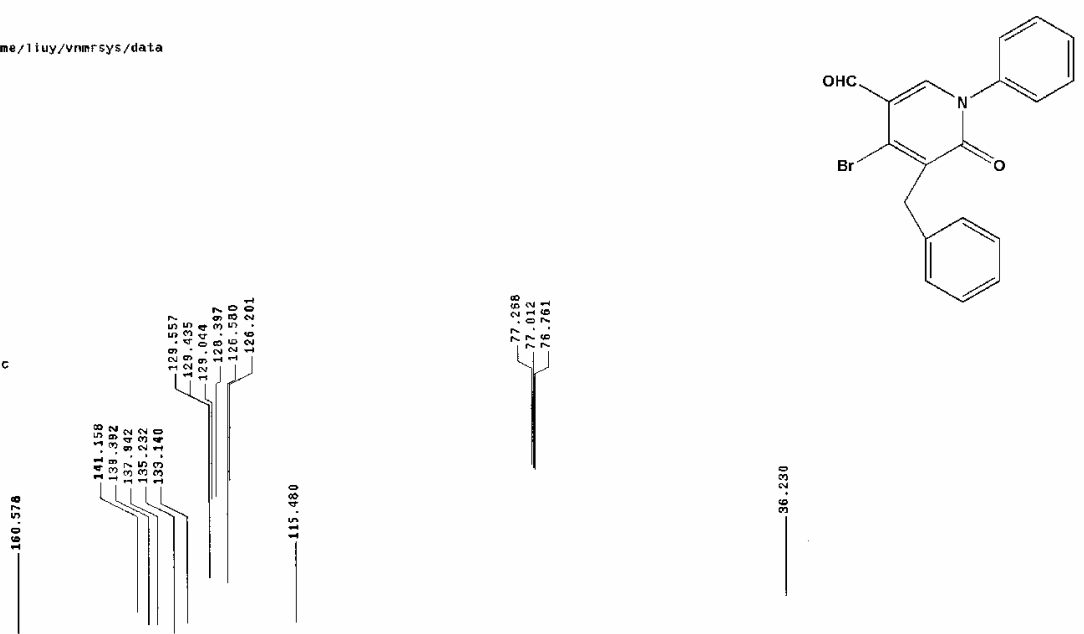

|
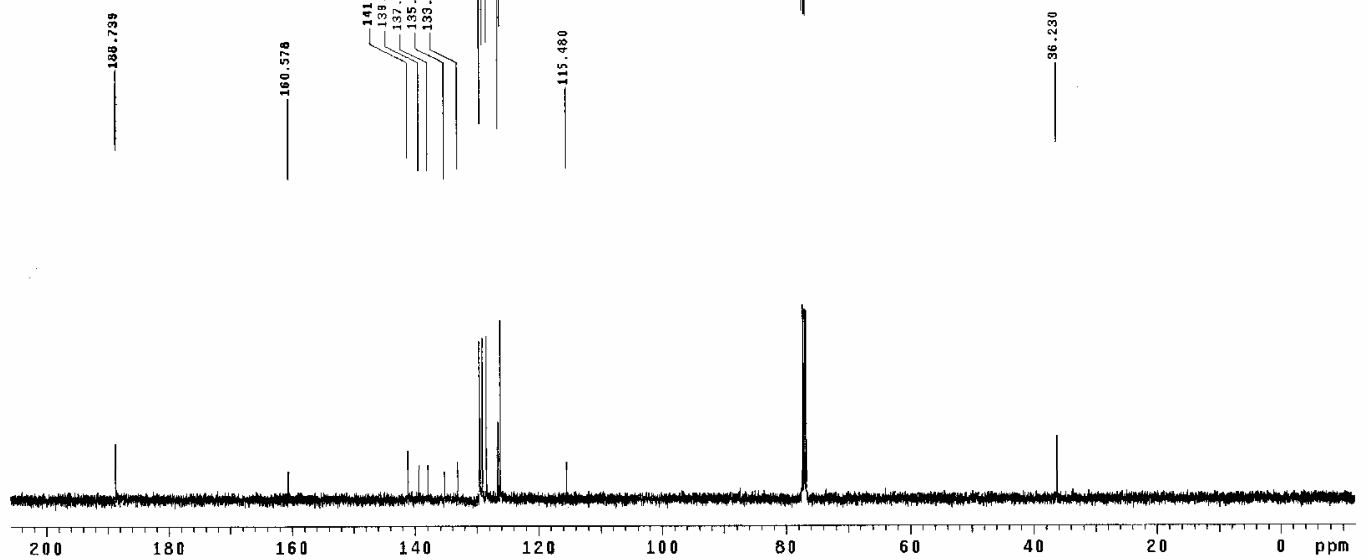
$2 b 1$

STARDARD PROTON PARAMETERS

Archive di rectory: /export/home/liuy/unnir sys/data

Pu7se Sequence: \$2pul

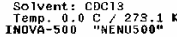

Relax. delay 1.000
Pulse

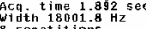

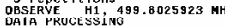

FT size 6556
Total time 0 min, 23 sec
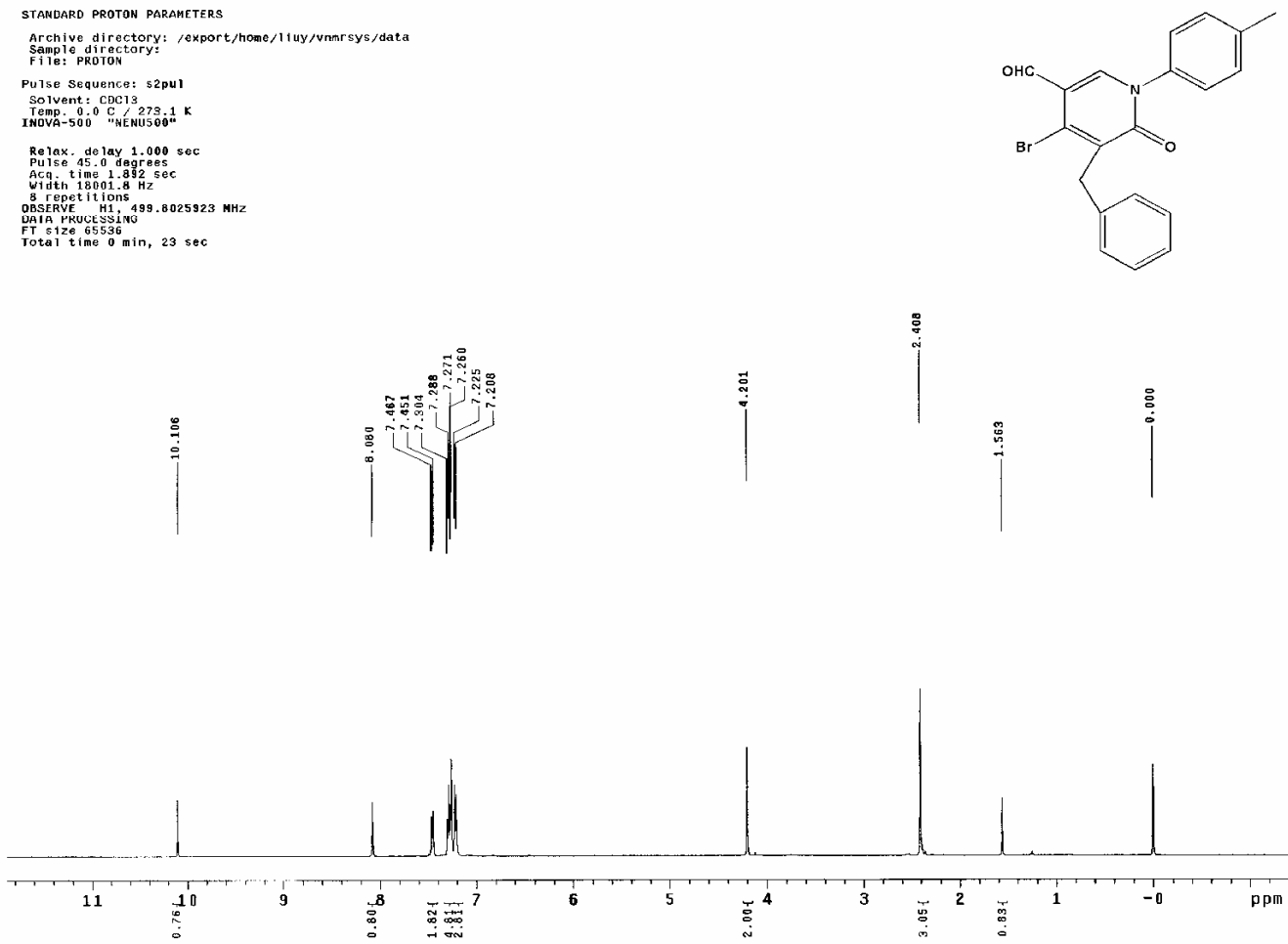

STAHDARD CARBON PARAMETERS

Archive directory: /export/home/liuy/vnmrsys/date
Sample directory:

Puise Sequence: $\mathrm{s} 2 \mathrm{pu}$

3oivent: $\operatorname{coc} 13$
Temp. $7.5 \mathrm{C} / 280.6 \mathrm{~K}$

Relax. delay $1.000 \mathrm{sec}$
Pulse 45.0 degrees

width $47990.4 \mathrm{~Hz}$

S4 repetitions
OBSERE C13, 125.6759679 HHz
DECOUPLE HA 499.8050905 HHz

rower 40 at
on dur ing acquisition

off during delay
Wal $z-16$ modulated

I ine troadering $1.0 \mathrm{~Hz}$

FT 5 jize 131072 ?
Total time $6 \mathrm{hr}, 24 \mathrm{~min}, 53 \mathrm{sec}$

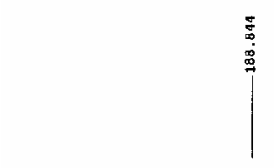

$\frac{10}{8}$

îj
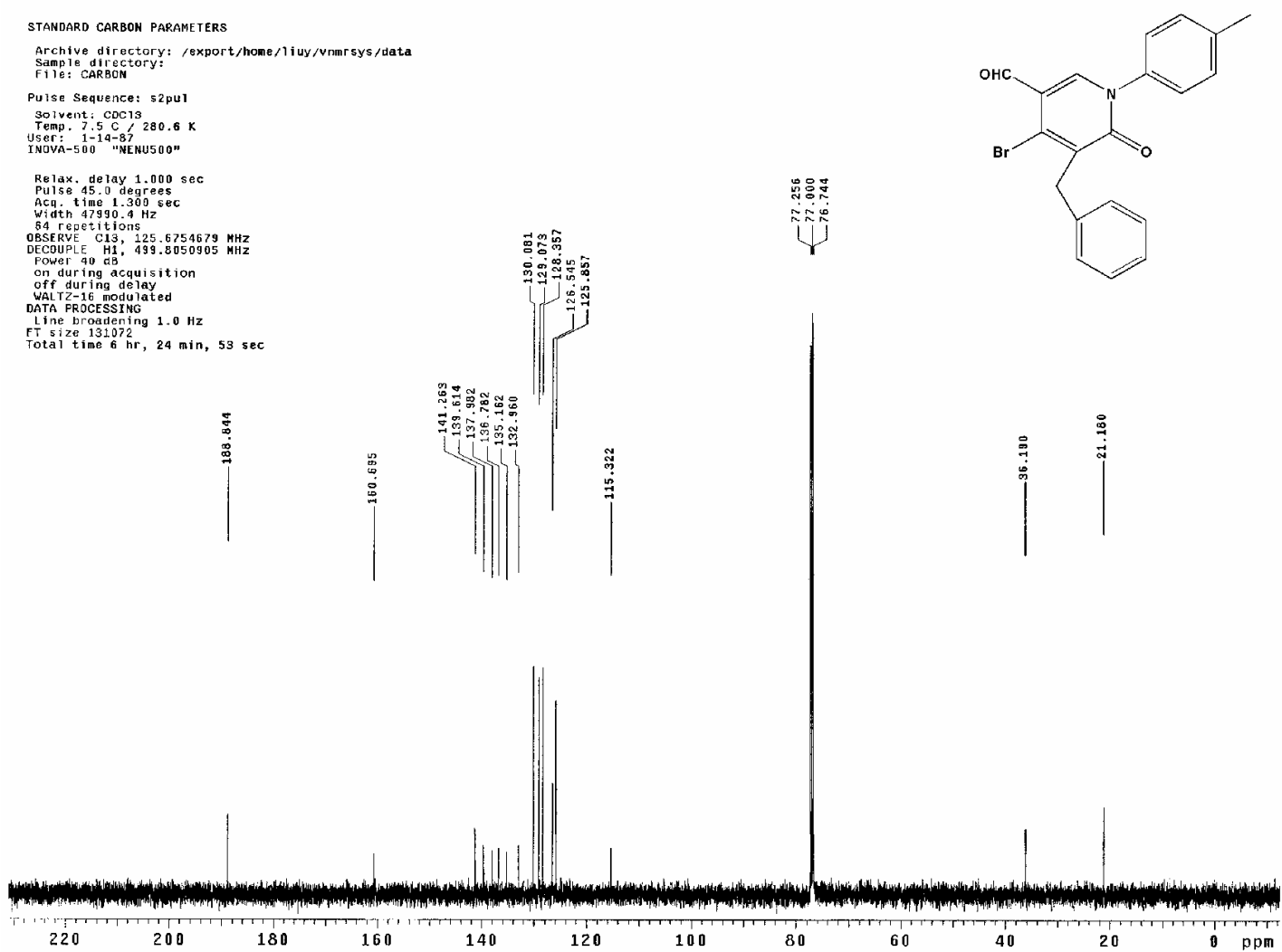
STANDARD PROTON PARAMETERS

Archive directory: /expart/home/liuy/vnmrsys/data

Pulse Sequence: szpu

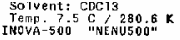

Relax. delay $1.000 \mathrm{~s}$
Pulse 45.0 ye grees

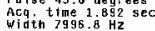

o repetitions

FT 5 ize 65536
Total tima $0.1 n, 23$ sec
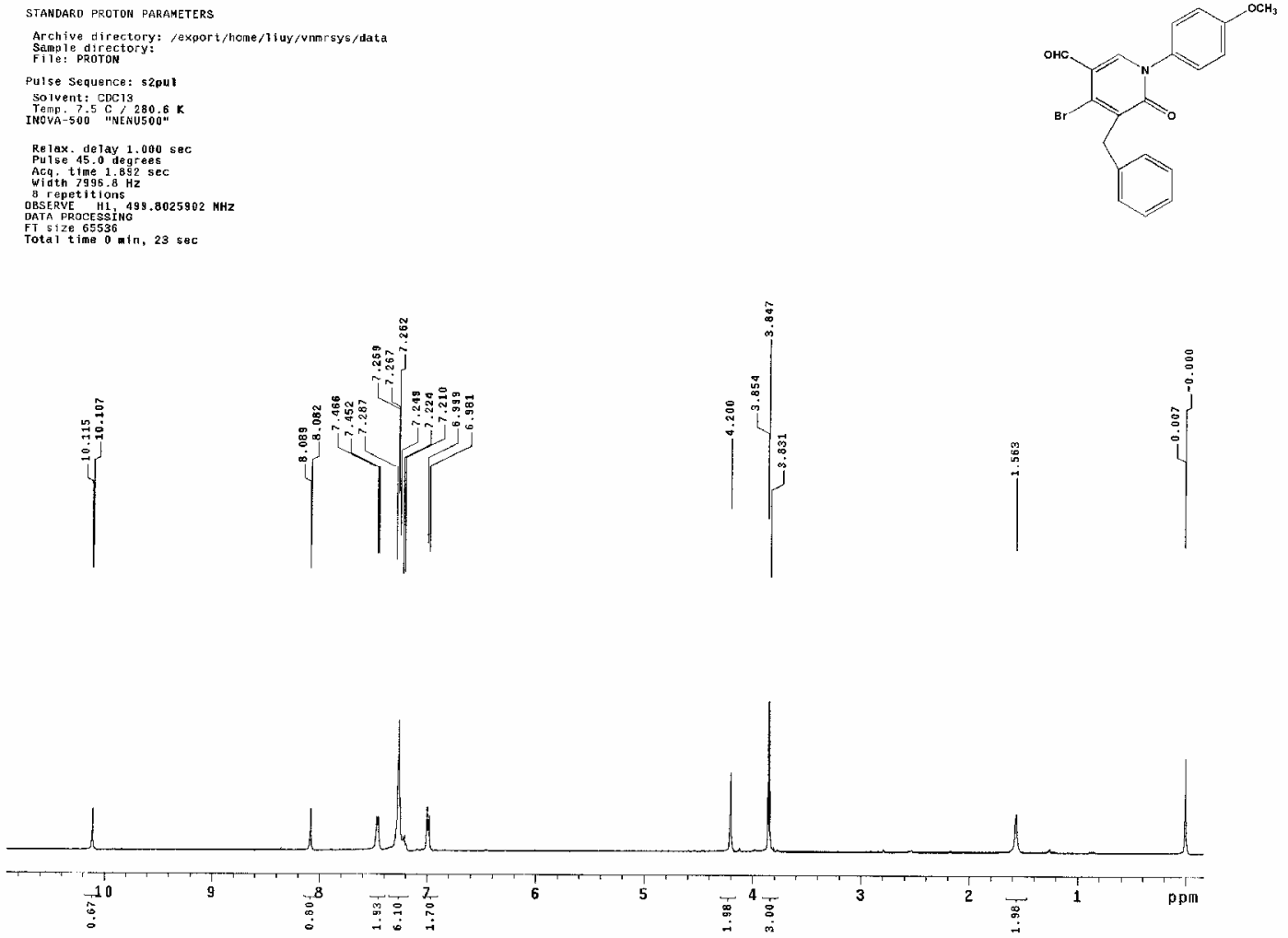

STANDARD CARBon PARAMETERS

Archlve di rectory: /export/home/rluy/unm rsys/data
sample directory:

Sample directory
File: CARBON

Pulse Sequence: $\mathbf{5 2 p u}$

Sulvelli: $\operatorname{CDC} 13$
Temp. $7.5 \mathrm{C}$. 280.6

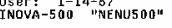

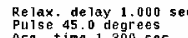

Acg time 1.300
witgh $47990.4 \mathrm{~Hz}$

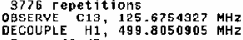

and

oner dur ing acquisition

off during de lay
waLTZ-16 modtulat

Line broadening $1.0 \mathrm{~Hz}$
FT 5 size 131072
Total time $6 \mathrm{hr}, 24 \mathrm{~min}, 53 \mathrm{sec}$
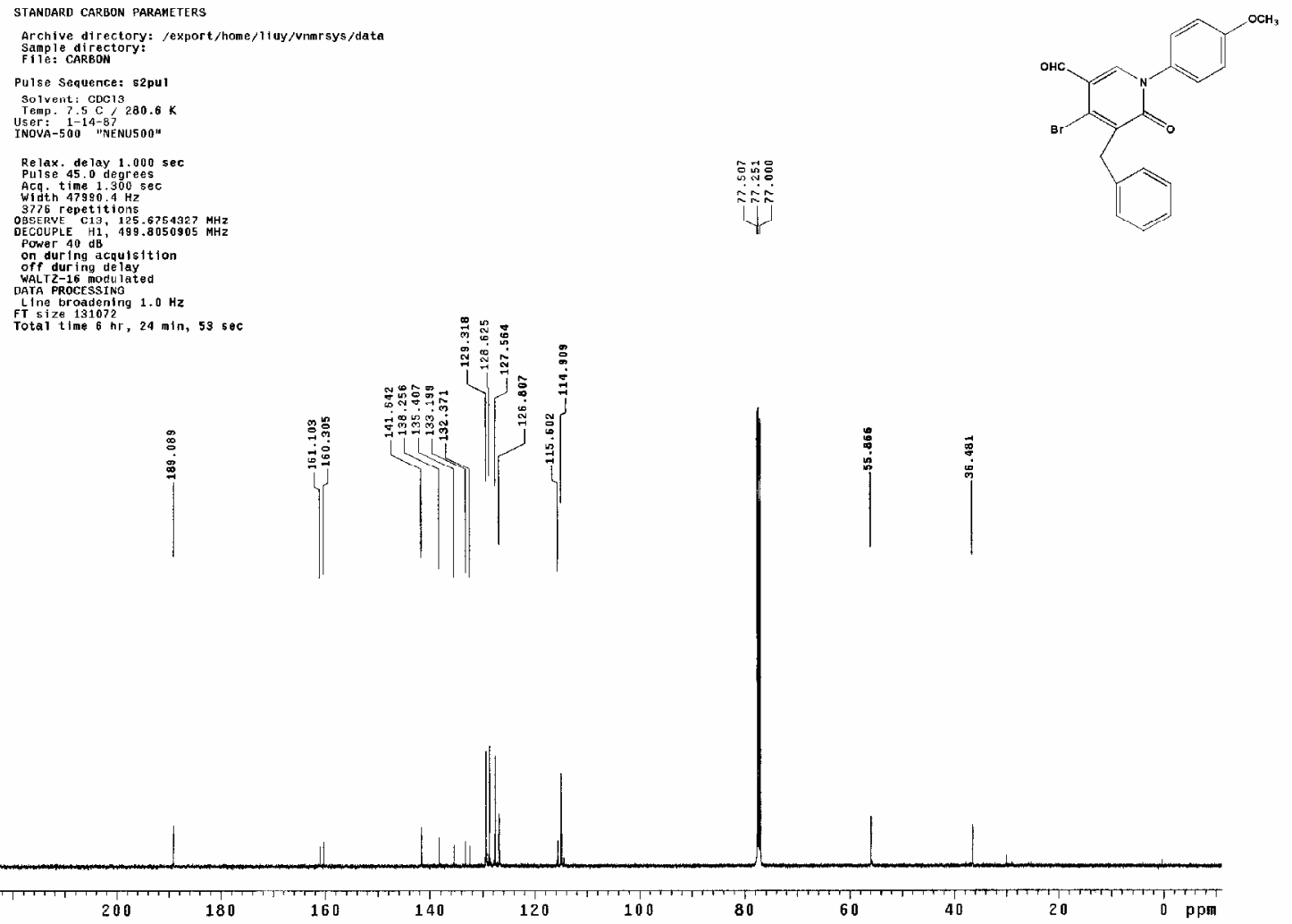
standard proton parameters

Archlue directory: /export/home/liuy/vnmrsys/date
Sample difrectory:

m.

Puise Sequence: s2put
Solvest: CDC 13 s
Temp. 0.0 C 273.1

Re7ax. delay $1.000 \mathrm{sec}$

Pulse 45.0 degrees

S repetitions
oBSERE

FT $51 z e, 6536$
rotal tine $0 \mathrm{~min}, 23 \mathrm{sec}$
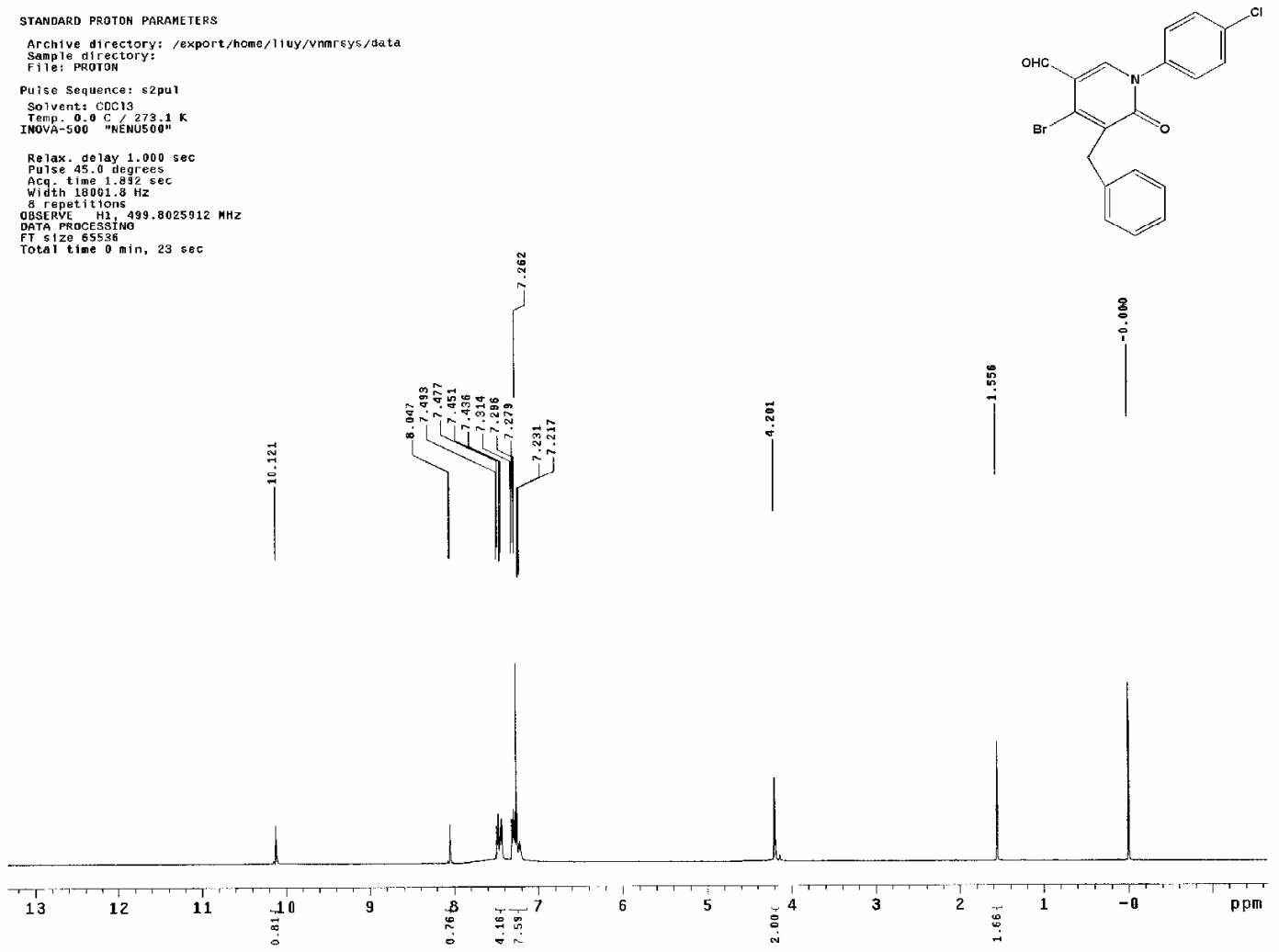

StamDard CARBon PARAMETERS

Archlye directory: /export/mome/liuy/unnrsys/data
Sample directory:

rinor cartect

Solvent: $\operatorname{coc} 13$

U⿴囗十)

Relax. de lay $1.000 \mathrm{sec}$

Pulse 45.0 degrees

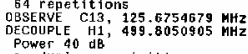

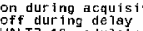

$\begin{array}{ll} & \\ & \end{array}$

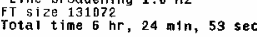
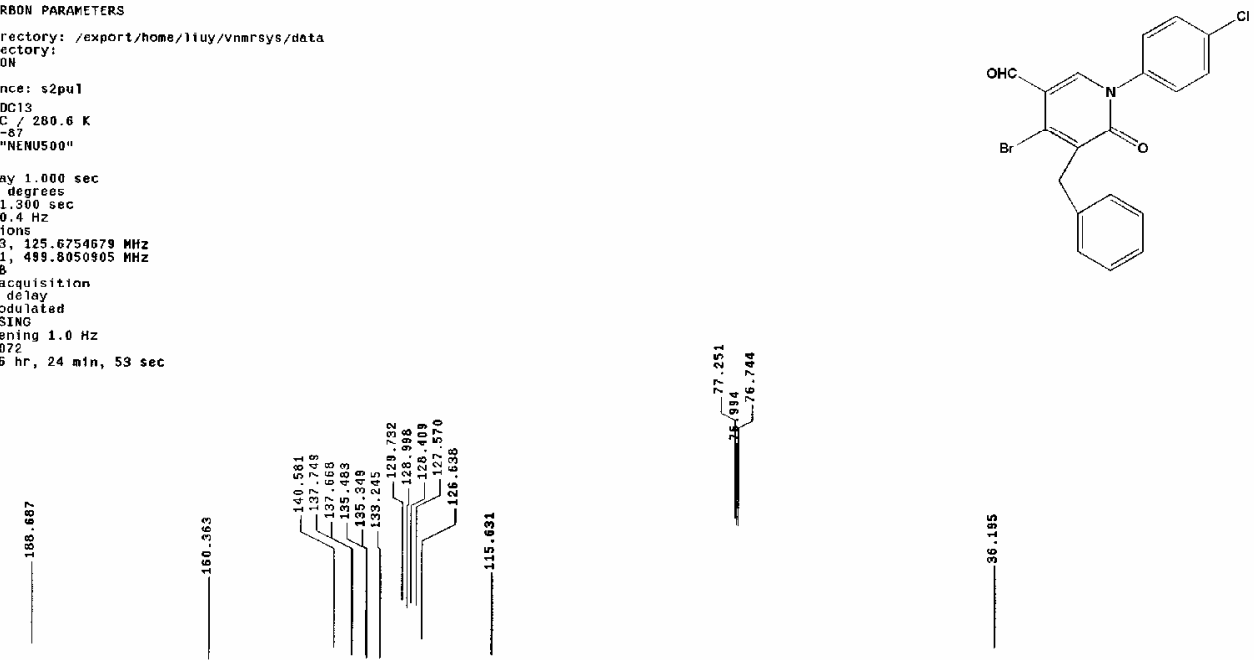


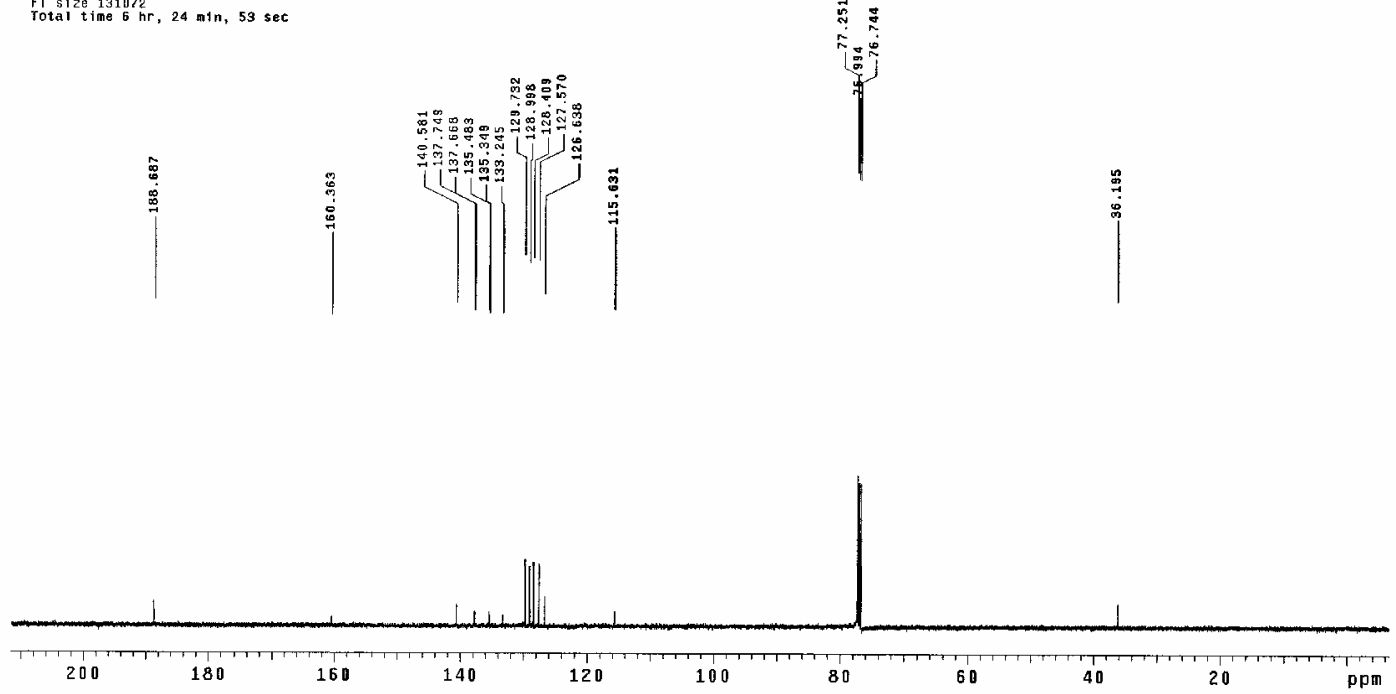
Tamoard proton paremeters

Archive directory: /export/home/liuy/unmorsys/date
Sample dirfectoryi

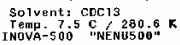

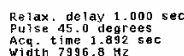

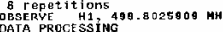

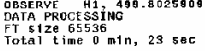
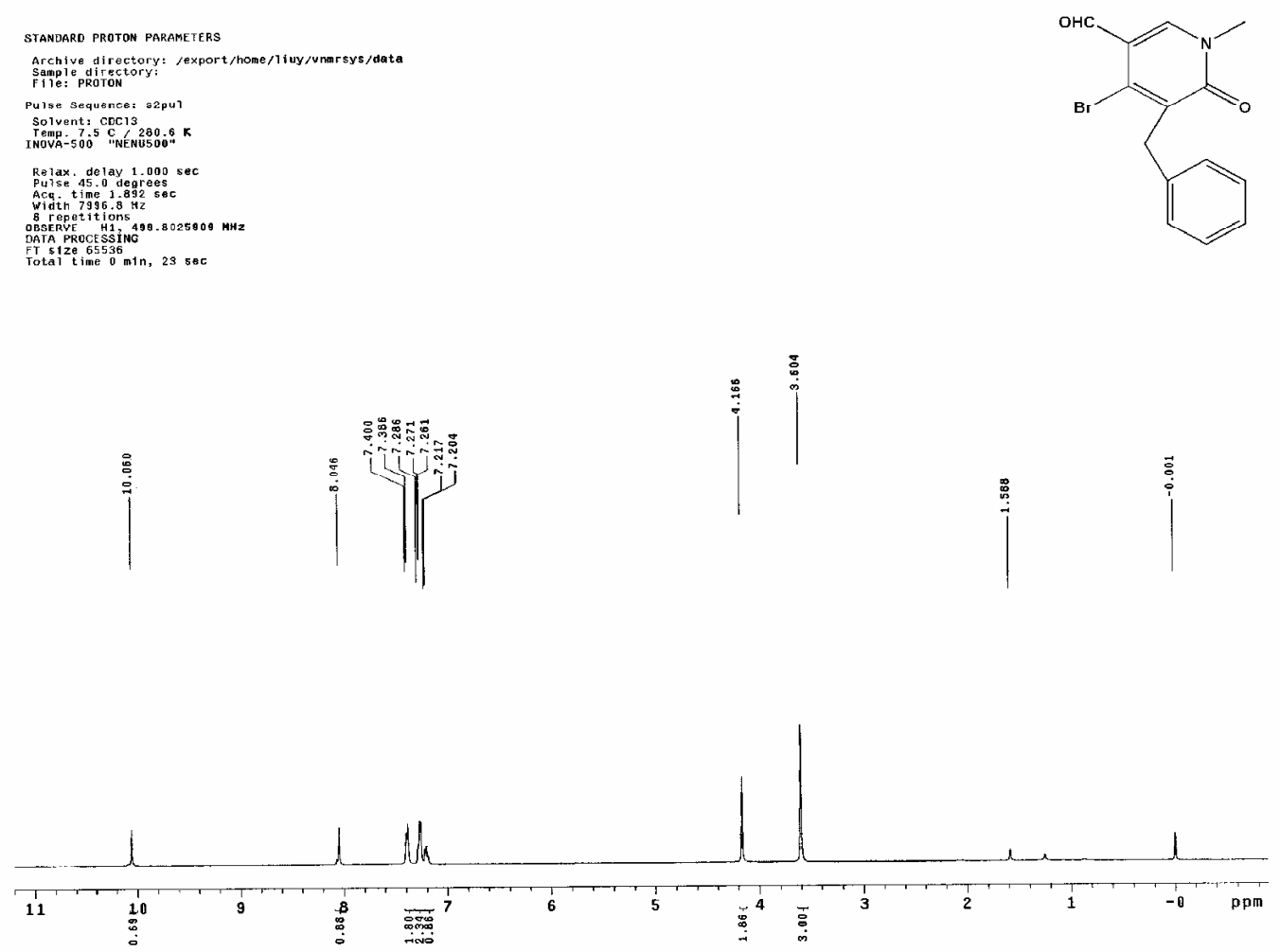

STANDARD GARBON PARAMETERS

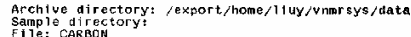

Pulse Saquence: s?nu

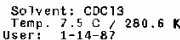

INOVA-500 "NE NU500"

Pulse 45.0 de drees

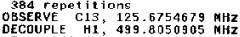

oner 40 in

WALTZ-16 modul ated
WATA PROCISSING

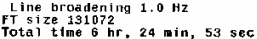
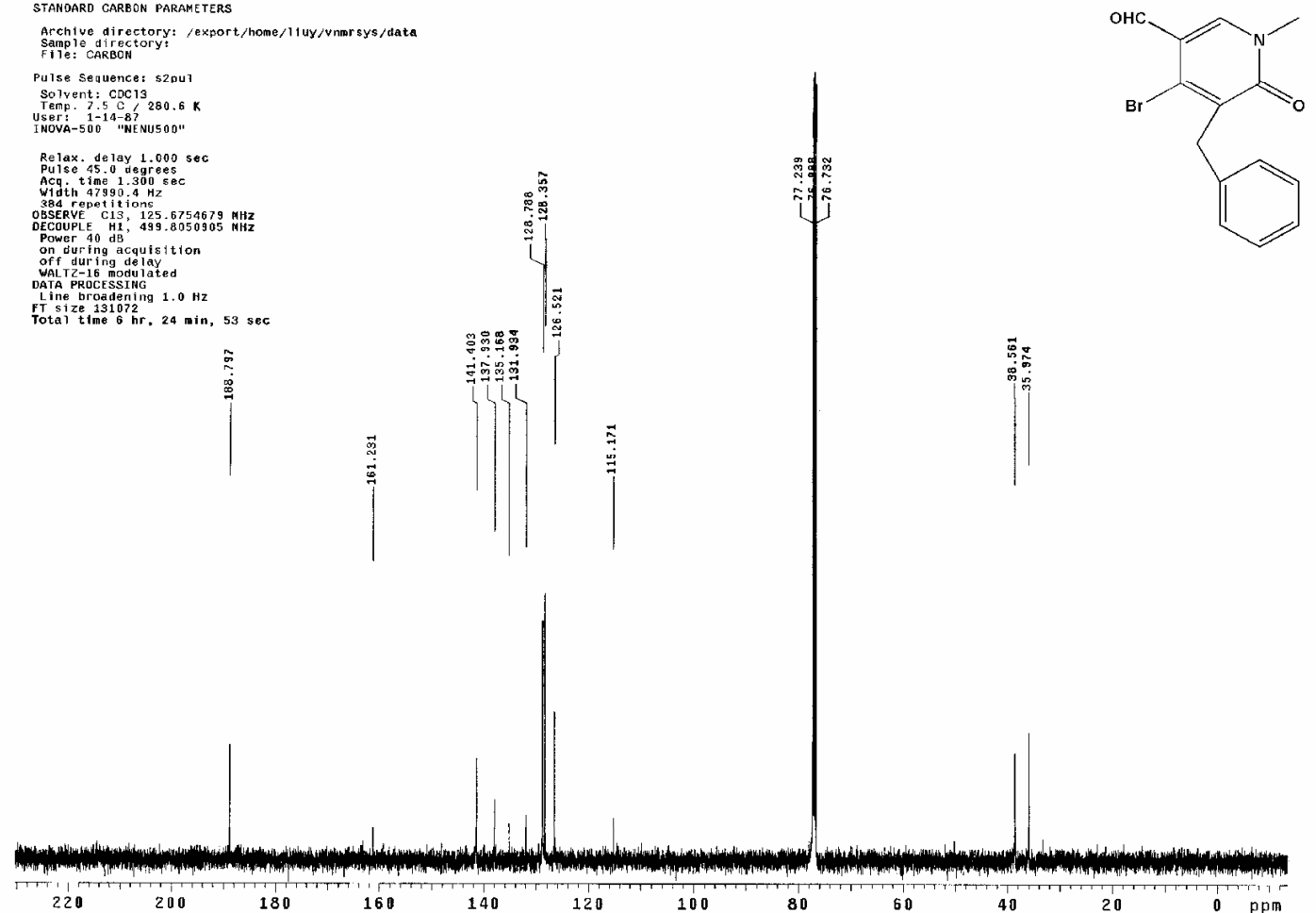
STANDARD PROTON PARAMETERS

Archive directory: lexport/home/liuy/vruarsys/dat

Pulse

Pulss Sequence: $52 \mathrm{pu}$
Solvent: $\operatorname{coc} 13$

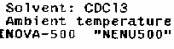

Relax. delay $1.000 \mathrm{sec}$
Pulse 45.0 degrees

Aca. time 1.8925

OESERVE H1, $499.8025901 \mathrm{MHZ}$

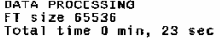
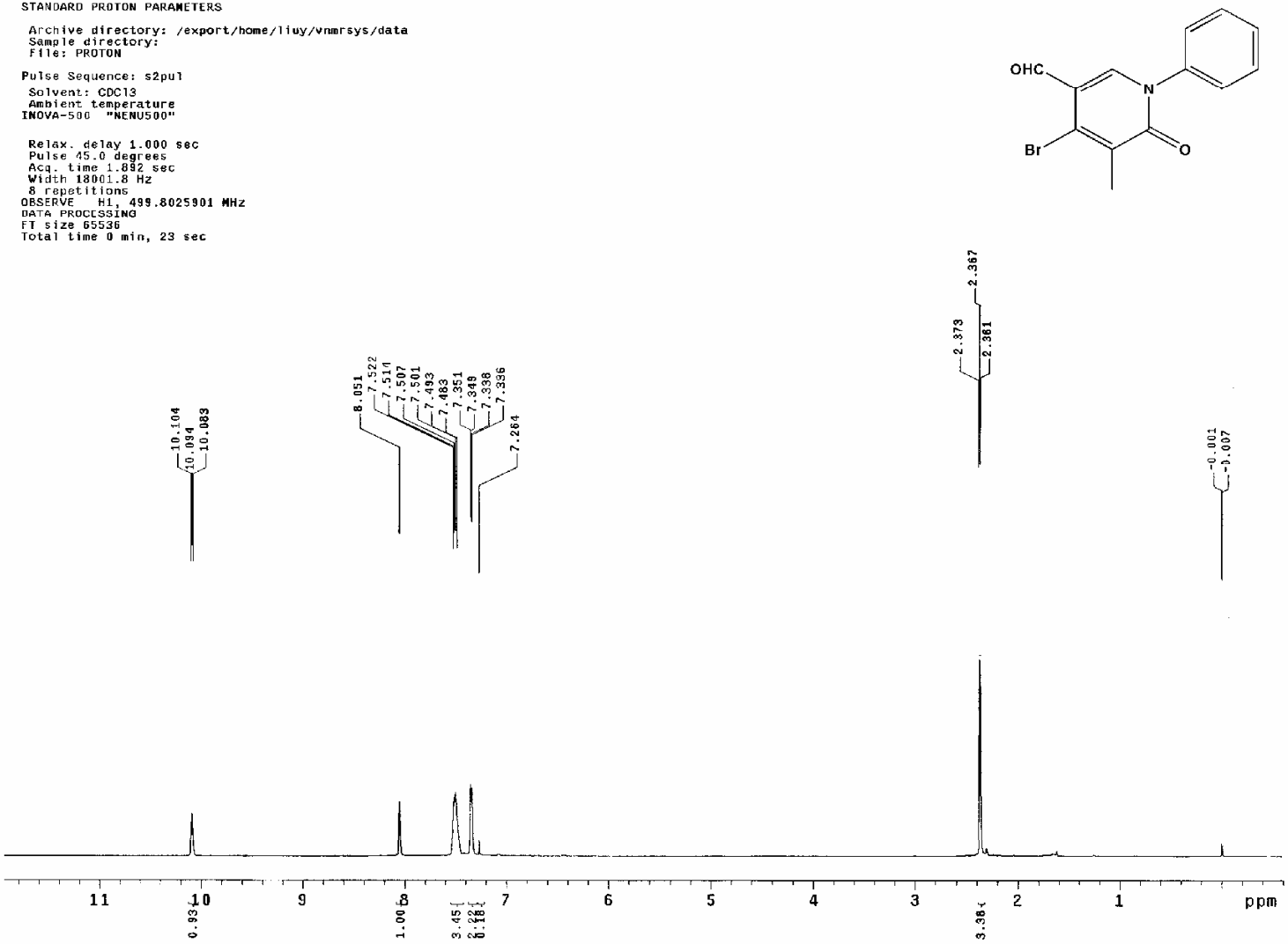

STANDARD Carbon PARANETERS

Archive directory: Jexport/mome/31uy/vnarsys/data

Pulse sequence: szoul

Solvent: CDC13 $300.6 \mathrm{~K}$

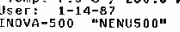

Relax. delay 1,000 soc

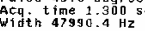

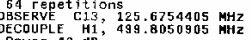

Power 40 dB
on durf ng acquisition

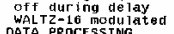

Line broadening $1.0 \mathrm{~Hz}$

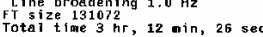

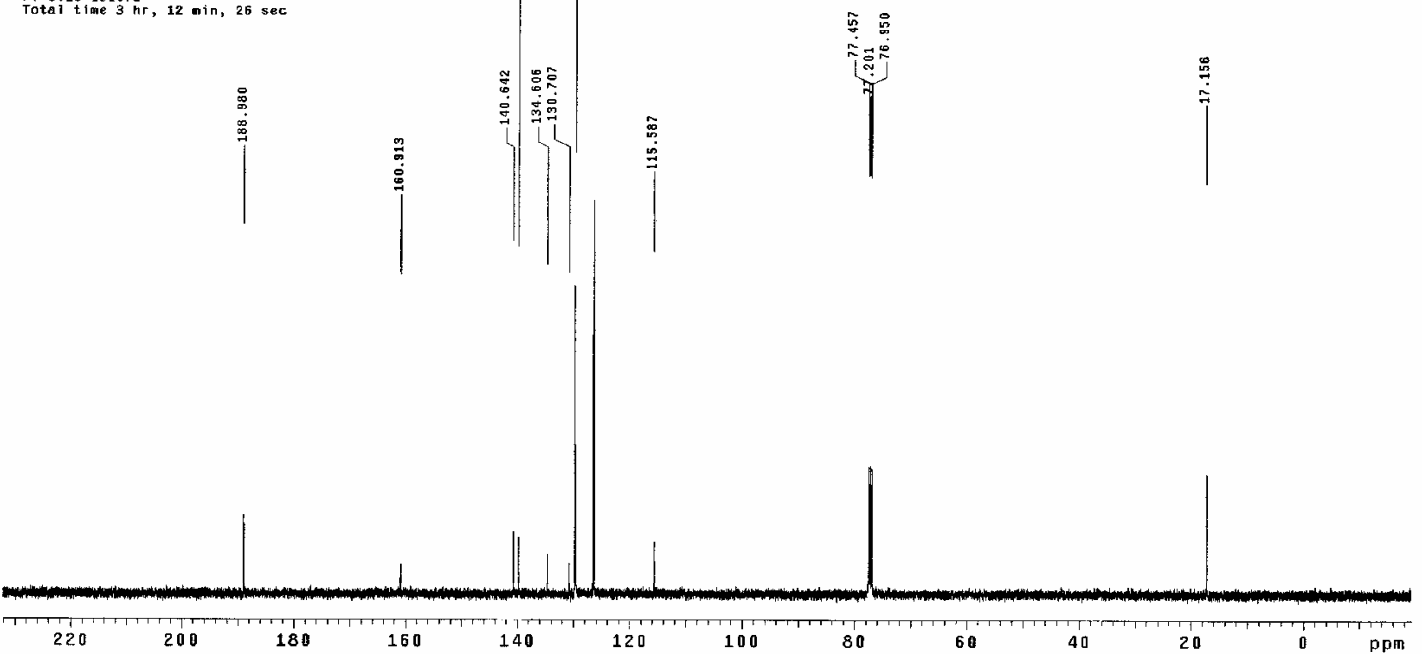


STANDARD PROTON PARAMETERS

Archive directory: /export/home/liuy/unmrsys/data
Sample directory:
File: PROTON

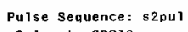

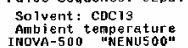

Relax. delay 1.000
Pulse 45.0 te

acy
Width
1 time 1800.892
Hz

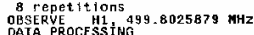

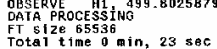

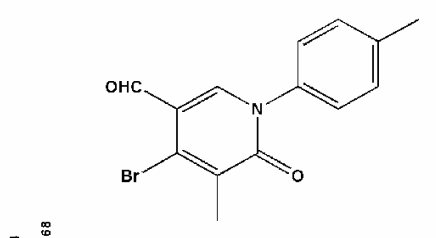

|
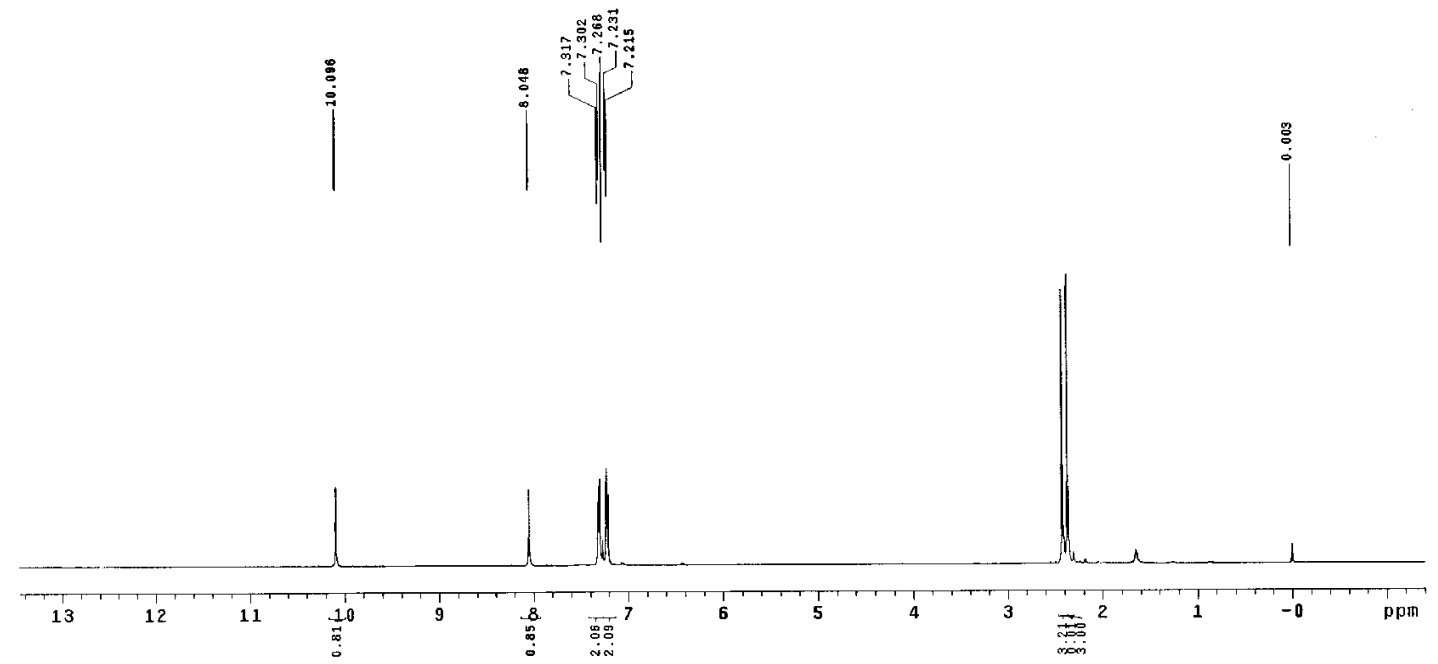

SIANOARD CARBON PARAMETERS

Archive directory: /export/home/liuy/unarsys/data
Sample directory:

Pulse Sequence: s2pul

Solvent: cocis $130.6 \mathrm{~K}$

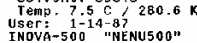

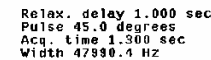

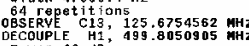

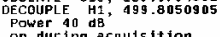

on dur ing acquisition
off dur ing delay
bai z

Line broadening $1.0 \mathrm{H}$

FT Sile 131072
Total tim $3 \mathrm{hr}, 12 \mathrm{~min}, 26 \mathrm{sec}$

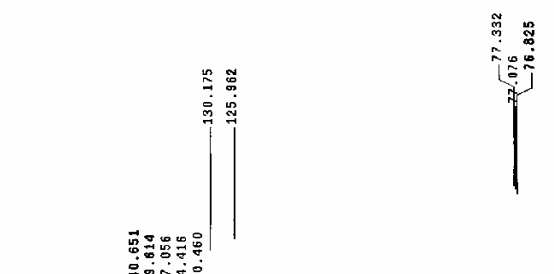

|
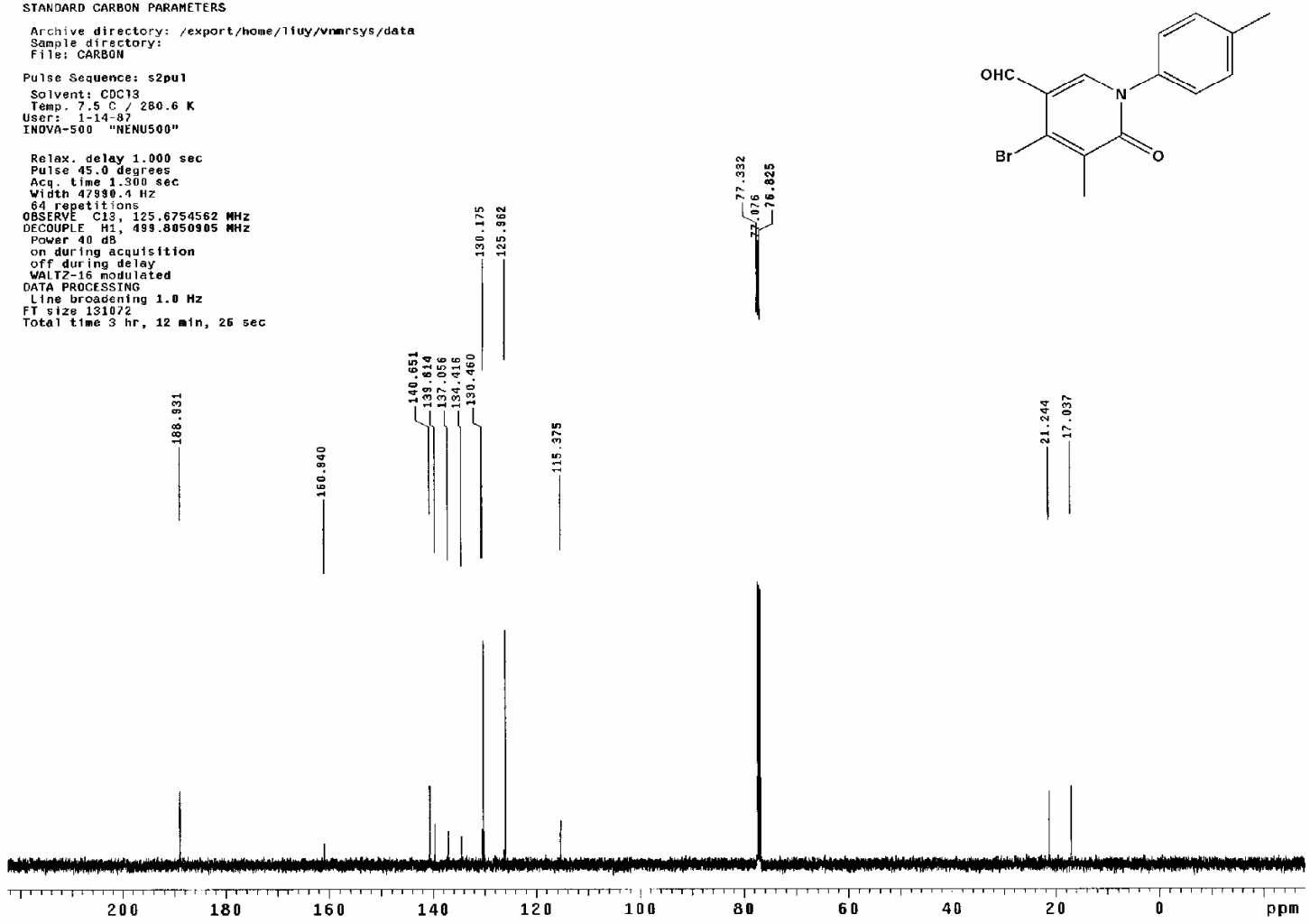
STAMDARD PROTOM PARAGETERS

Archive directory: /export/home/li uy/vnirsys/data
Sample directory:
File

Pulse Sequence: szpul

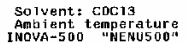

Relax- delay 1.000 sec

Acs 1 me $2.892 \mathrm{sec}$

A repetitions
BesERE
B.

FT stre 65536
Total tine $0 \mathrm{~min}, 23 \mathrm{sec}$
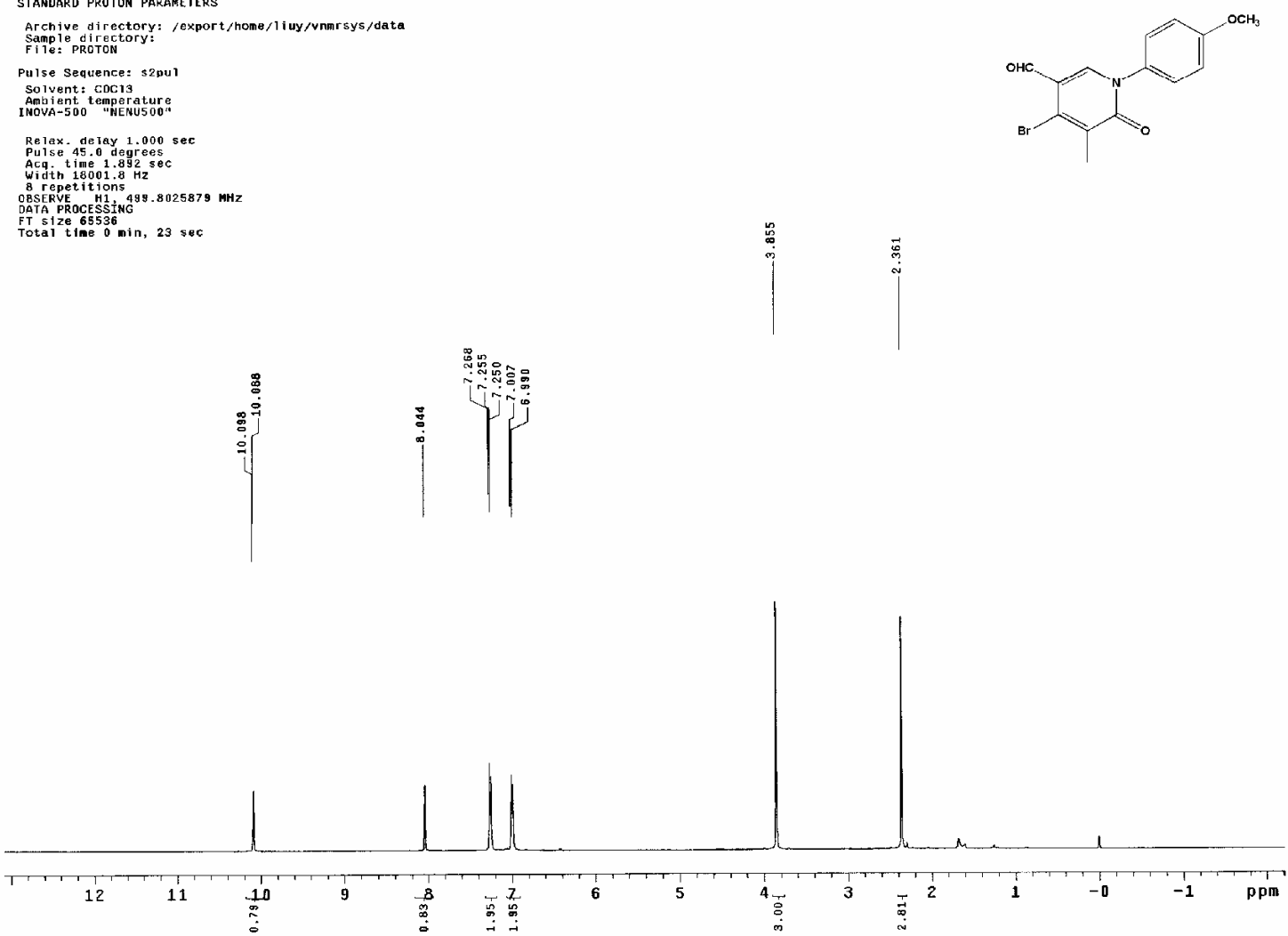

Carbon parameters

Archive directory: /export/home/1iuy/unmrsys/data
Sempe directory:
File: CARBOW

Pulse Sequence: szput

Solvent: $C D C 13$ ( $200.6 \mathrm{~K}$

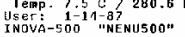

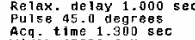

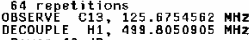

Power 40 dB

WAL T $2-16$ modulazed

Litine broadening $1.0 \mathrm{~Hz}$
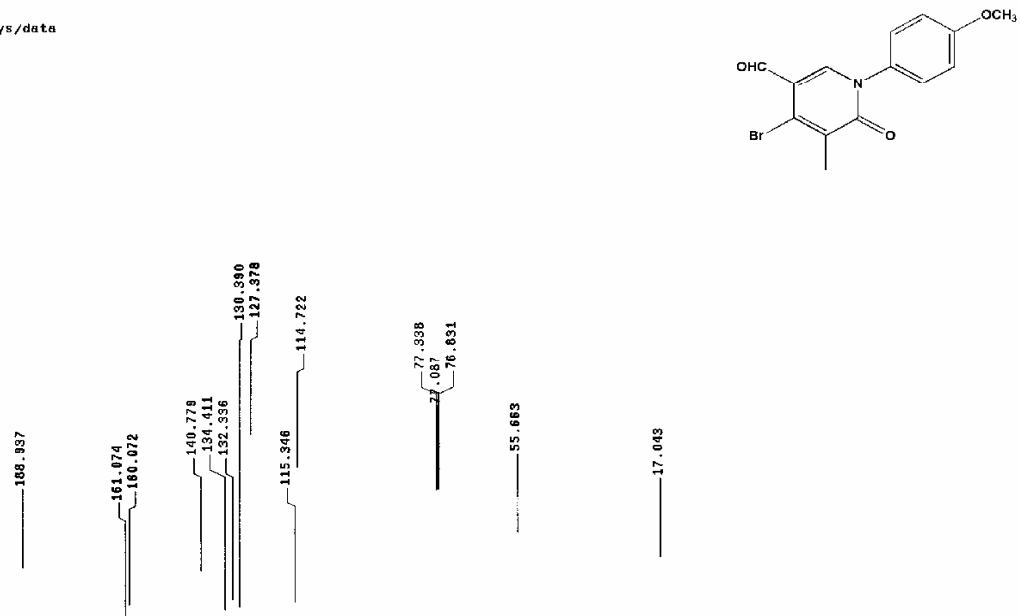

Fot size $131072012 \mathrm{~Hz}$
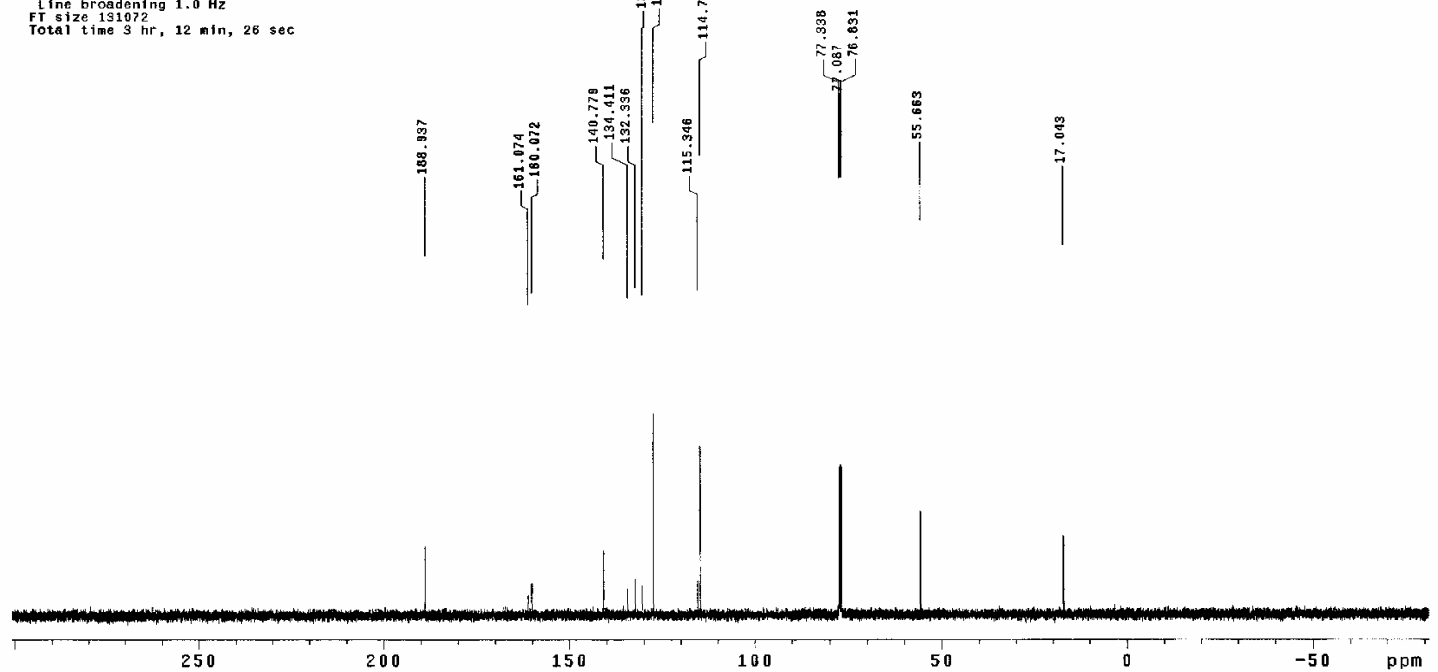
STANDARO PRDTON PARAMETERS

Archive directory: /export/home/liuy/vnimrsys/data
Sample directory:

Pulse seyueine: szpul

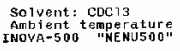

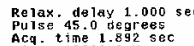

8 retetitions

102058

FT 5 ize 65536
Tota? time $0 \mathrm{~min}, 23 \mathrm{sec}$
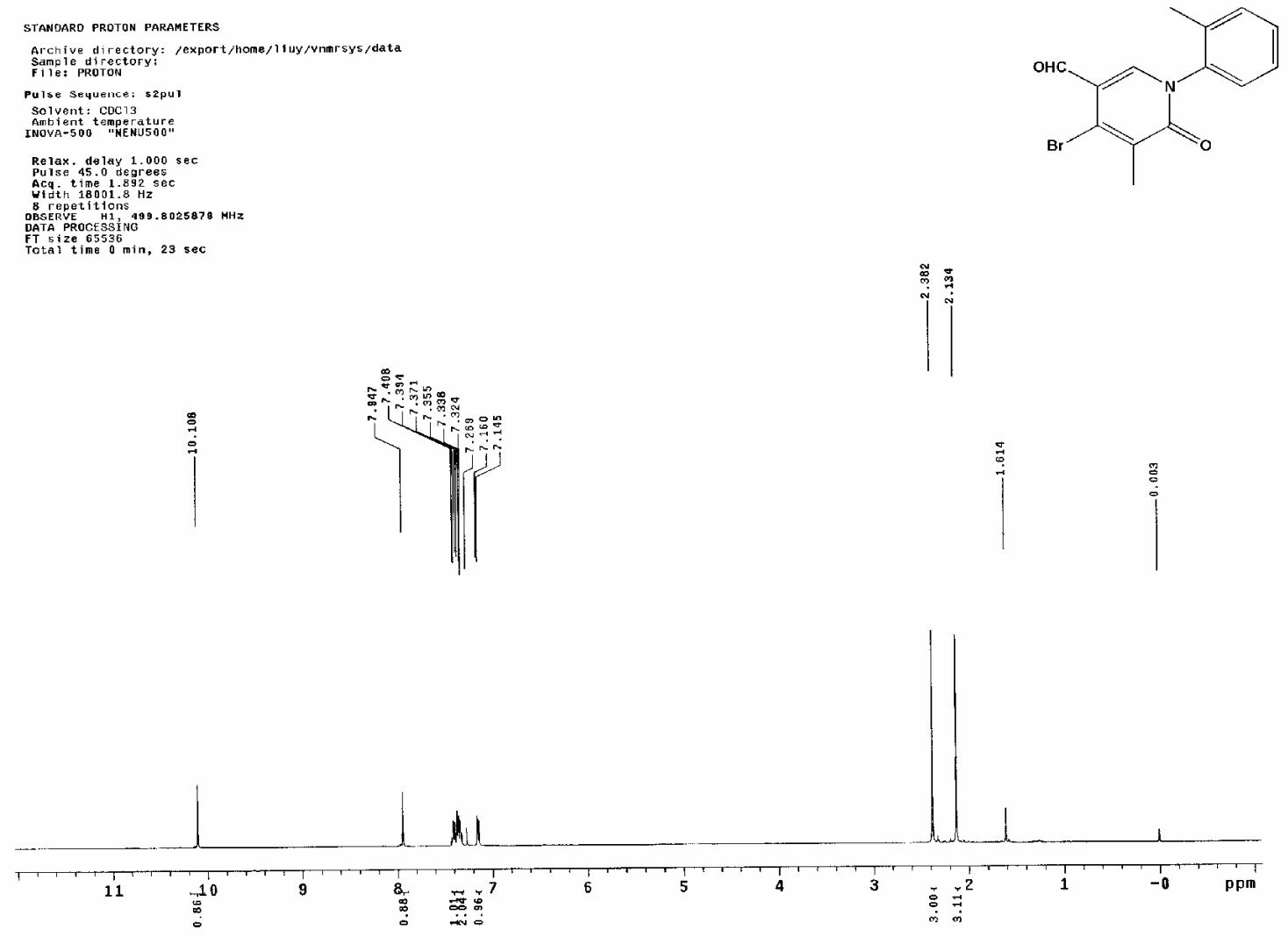

STANDARD CARBOW PARAMETERS

Archive directory: /export/home/1 luy/vnarsys/data
Sample directory:
File: CARBOH

Pulse sequence: 52pul

Solvent: $\operatorname{coc} 13$ Te $280.6 \mathrm{~K}$

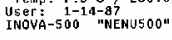

Re lax. delay $1.000 \mathrm{sec}$
Pulse 45.0 degres

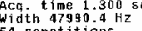

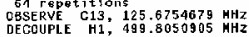

Power $40 \mathrm{~dB}$ -

on during acquisition

DATA PROCESS ING

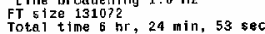
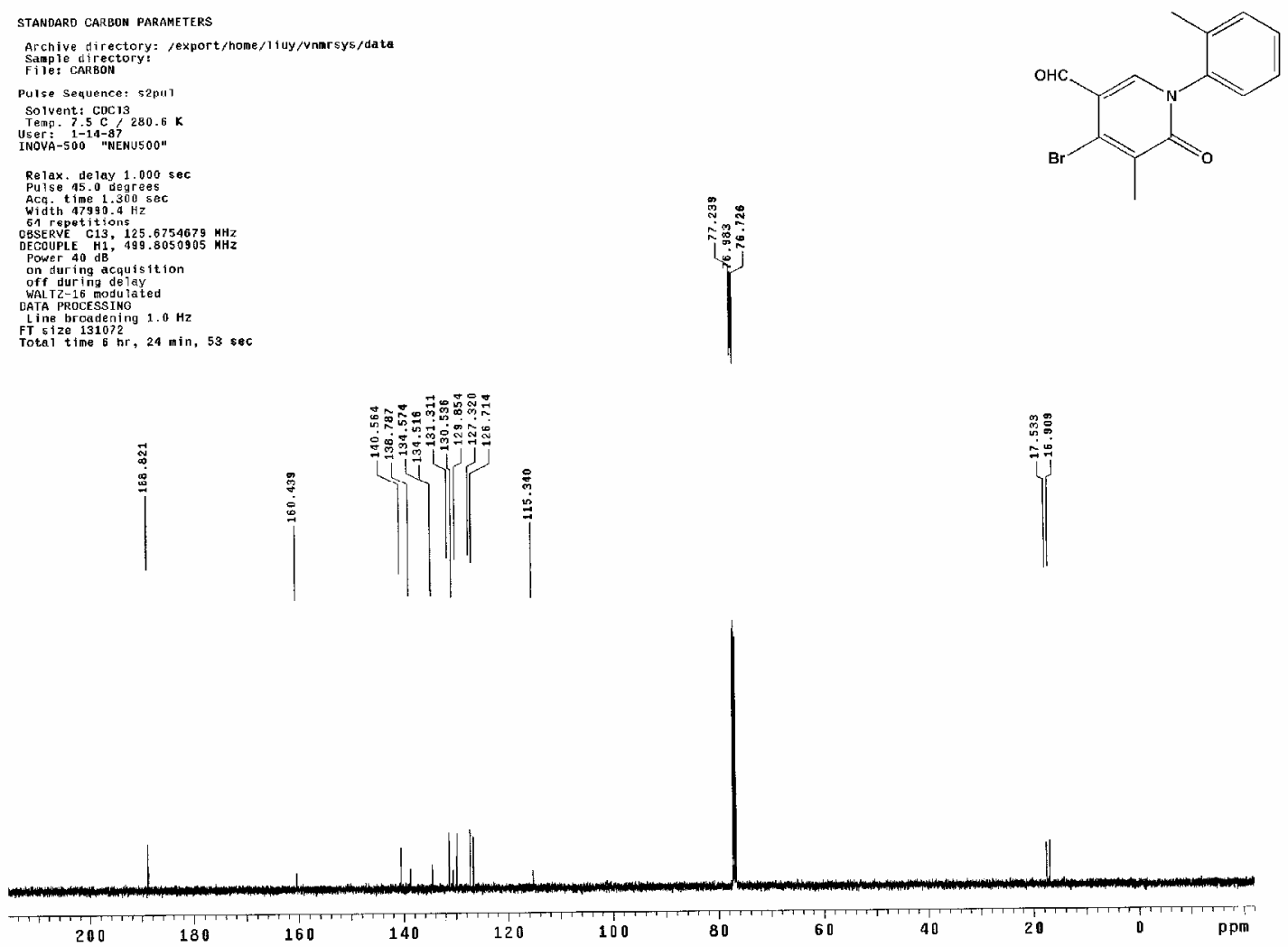
STANDARD PRoton PARAMETERS

Arehlue directory: /export/home/liuy/vnmrsys/data
sample directory:

Solvent: CDE 13 s

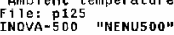

Relax. de lay 1.000 se

Acq.

8 repetitions
OSSERYE RY

DAAA PROCESSING
FT 5 ize 65536
Total time 0 .
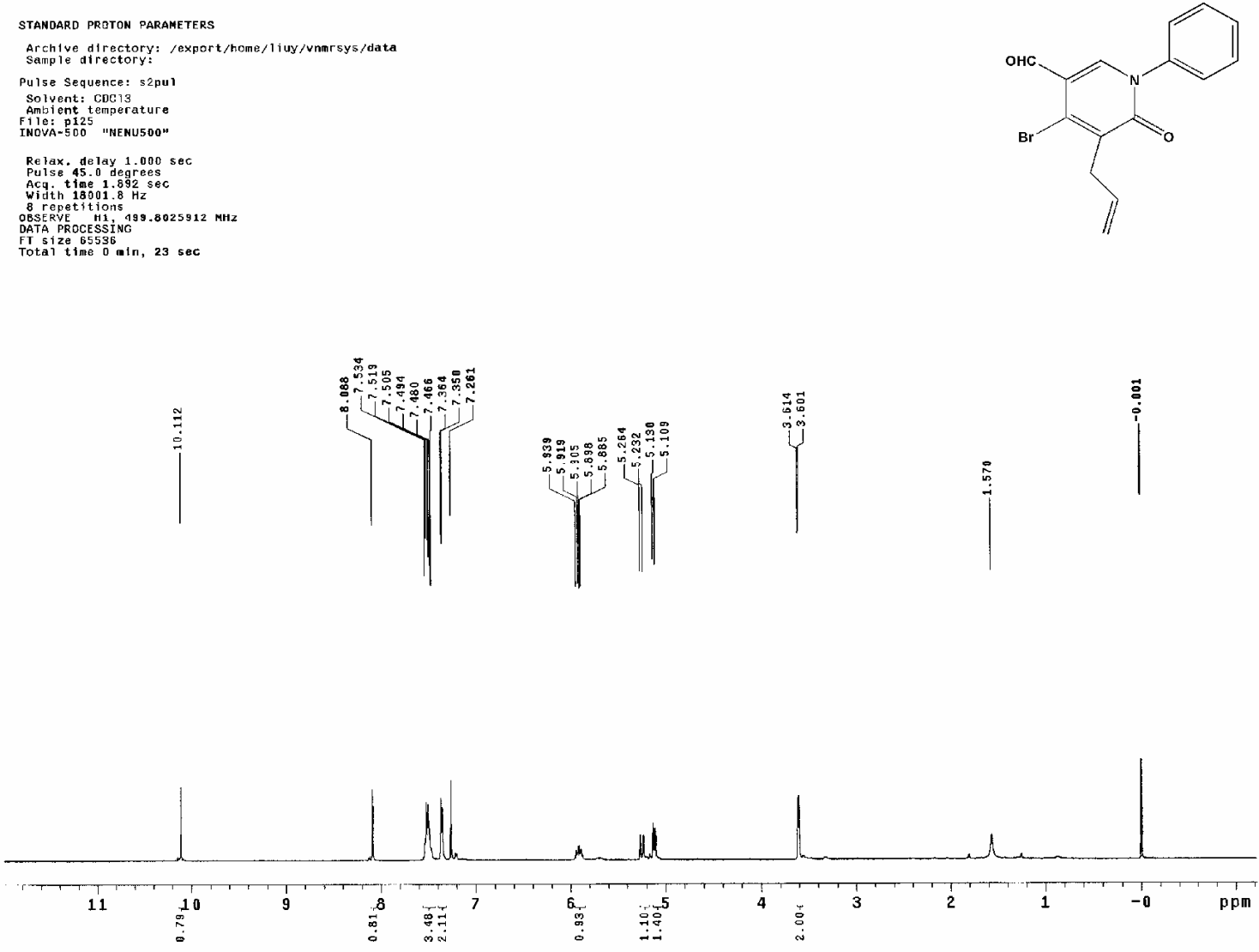

standaro carbon parameters

Archlve directory: /export/home/liuy/vnmrsys/date

ile: cakbon

Pulse sequence: s2pur

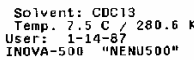

Relax. delay $1.000 \mathrm{sec}$
Pulse 45.0 degrees

Acy tine 1.300 sec

OSEREVE $13,125.6754405 \mathrm{MHz}$
OECOUPLE H1, 499.8050905 MHE

Power 40 dB
on dur ing acquisition

off during delay

DATA LCCESING $1.0 \mathrm{~Hz}$

Fol
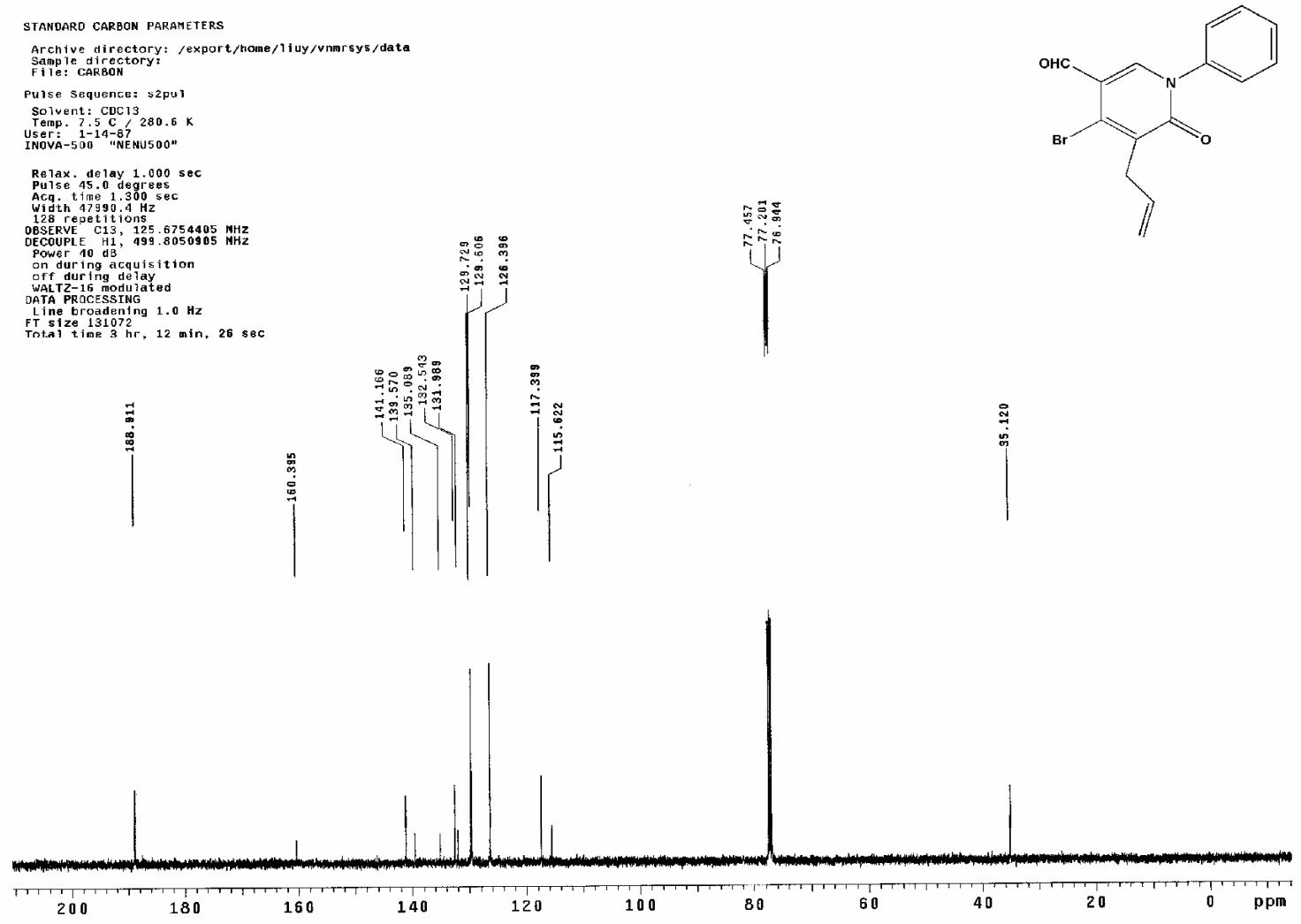
Standard proton parameters

Archive directory: /export/home/liu/vnarsys/data
Sample directory:
File poTol

ㄱ..ㄹ..ㄹ.

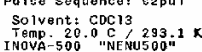

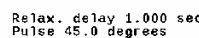

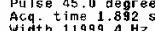

S repetitions 0 .

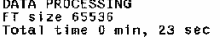
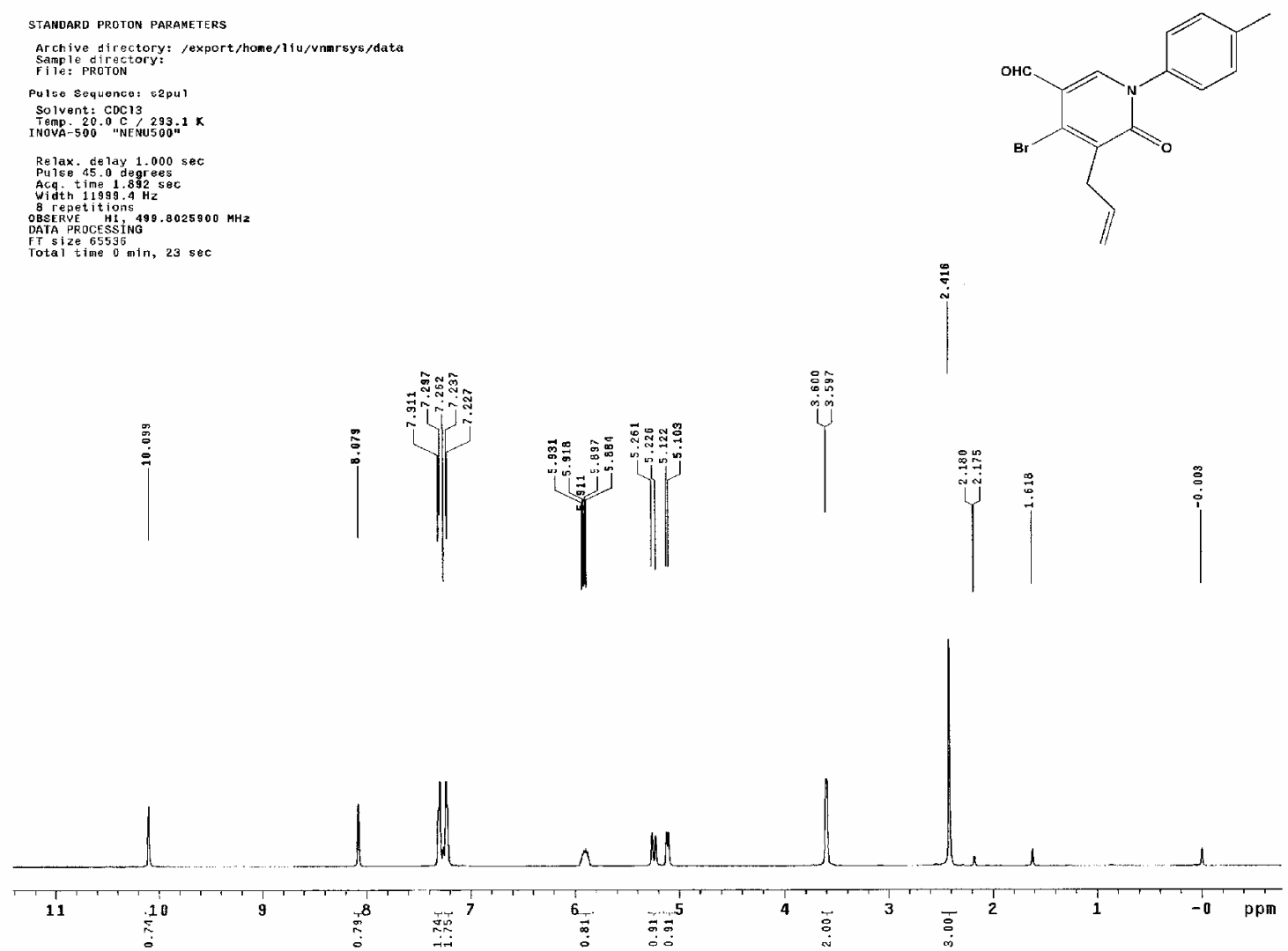

STAMDARD CARBon PARAME Ters

Archive directory: /export/homes 1 i uy/vmarsys/data
Samp le directory:
File carean

Pulse Sequence: s2pur

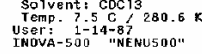

Relax. de lay 1.000 sec
Purse 45.0 degrees

Aca.
wime 1.300
widit $47990.4 \mathrm{~Hz}$

OBSERE C13, 125.6754679 AHZ
DECOUPLE H1, 499.8050905 HHZ

Power 40 de
on dur ing acquisition

Orf dur Ing delay
WAL
DOTZ

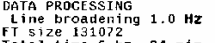

Fotaitime $6 \mathrm{hr}, 21 \mathrm{mtn}, 53 \mathrm{eac}$
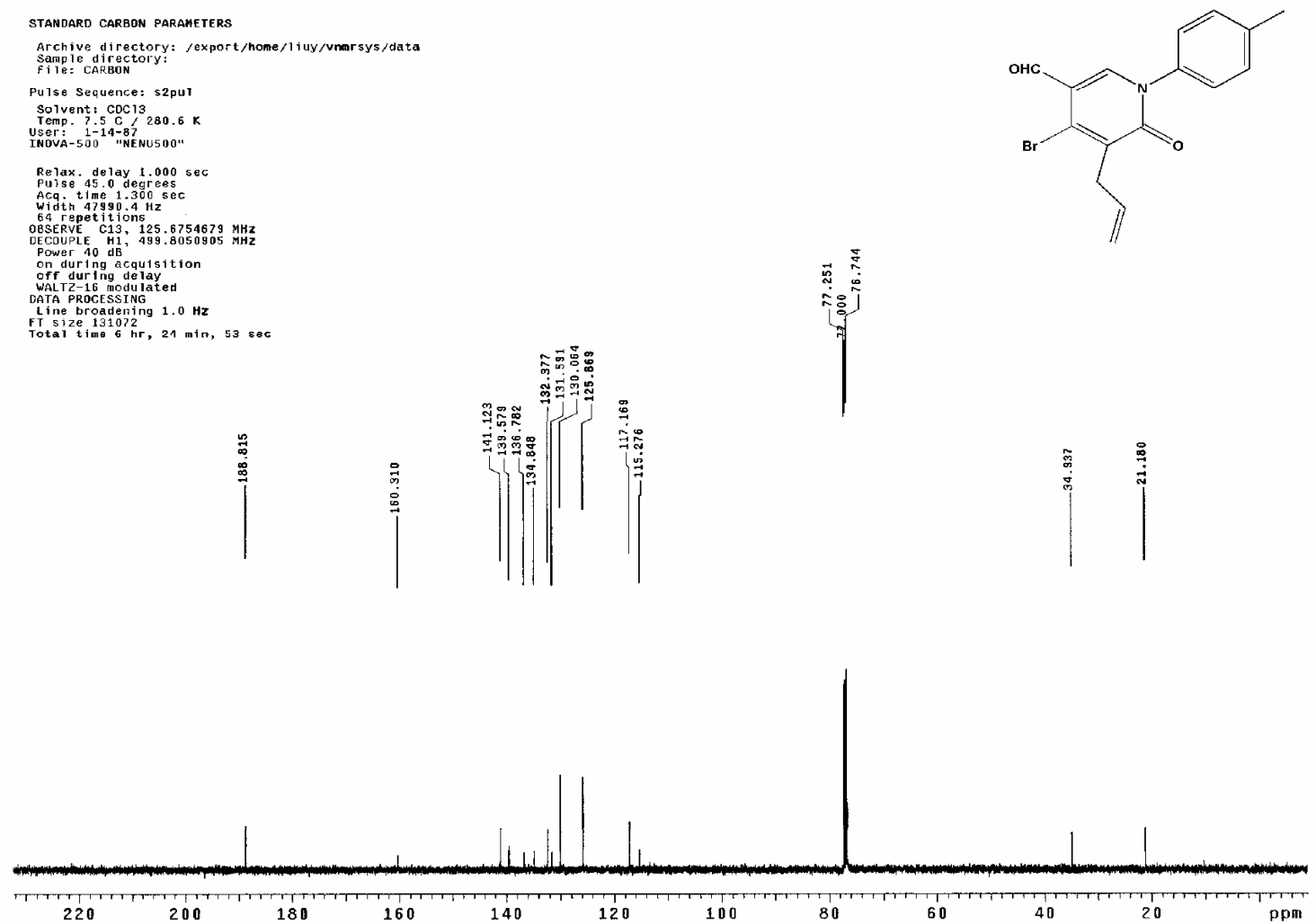
STANDARD PROTON PARAMETERS

Archive directory: /export/home/11 uy/vnmrsys/data
Sample pirectory:
Fils: PROTON

Pulse Sequence: szpul

Solvent: ${ }^{20 C 13} 28$

Relax. delay $1.000 \mathrm{sec}$

Pulse 45.0 degrees

Act time 1.892
Widt 18001.8
8

BSERVE H1. 499.8025909 HHZ

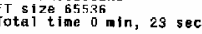
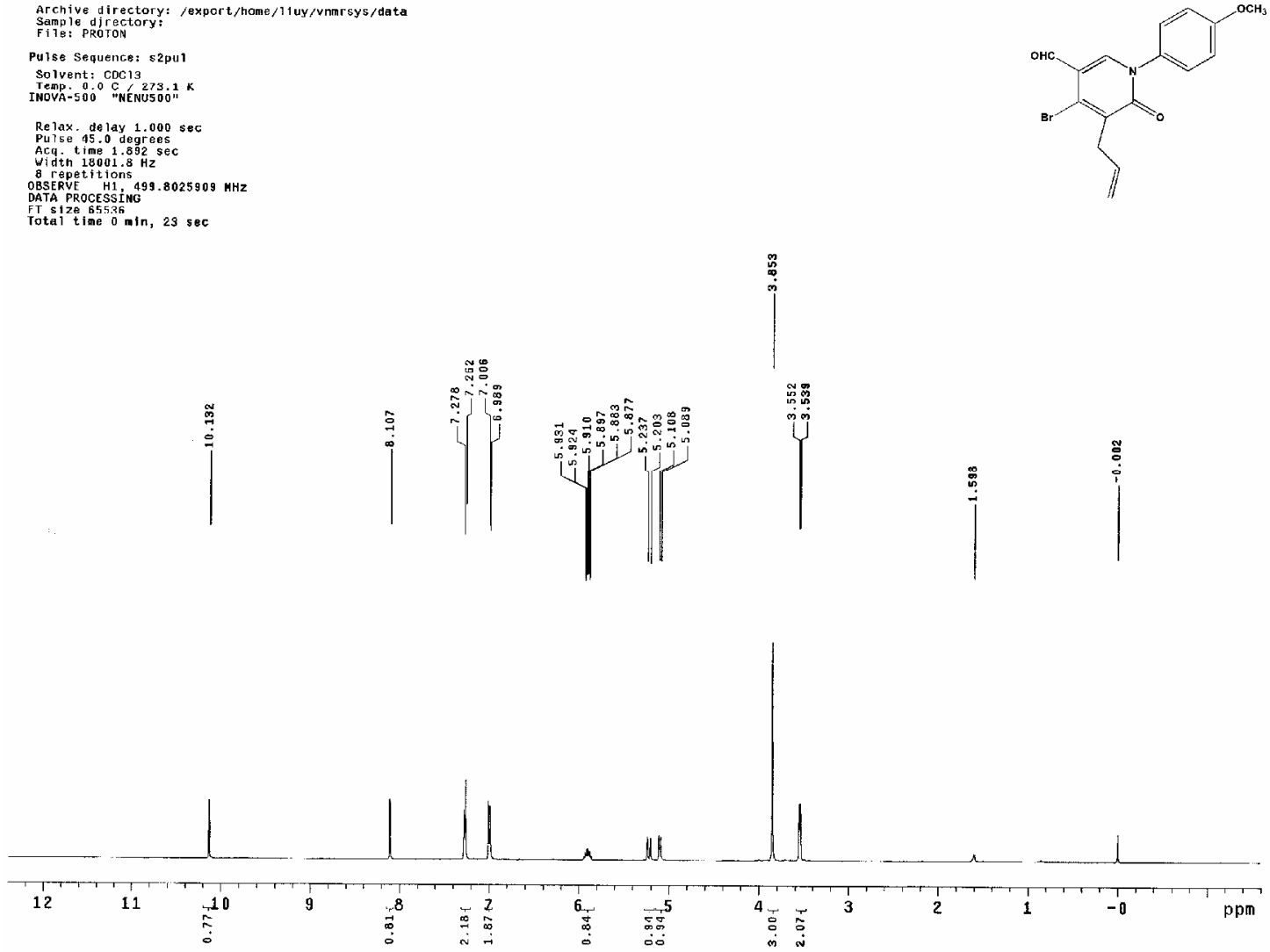

stanoard carbon parameters

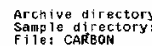

Pulso Sequence: s2pur

Solvent: $\operatorname{coc} 13$ s $80.0 \mathrm{~K}$

User. $1:-14-87$
INOVA-500 "NENUS00"

Relax. delay 1.000 soc

Acy time $1.300 \mathrm{sec}$

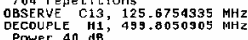

Power 40 dB
on dur ing acquisitio
off during delay

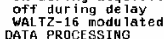

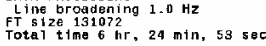
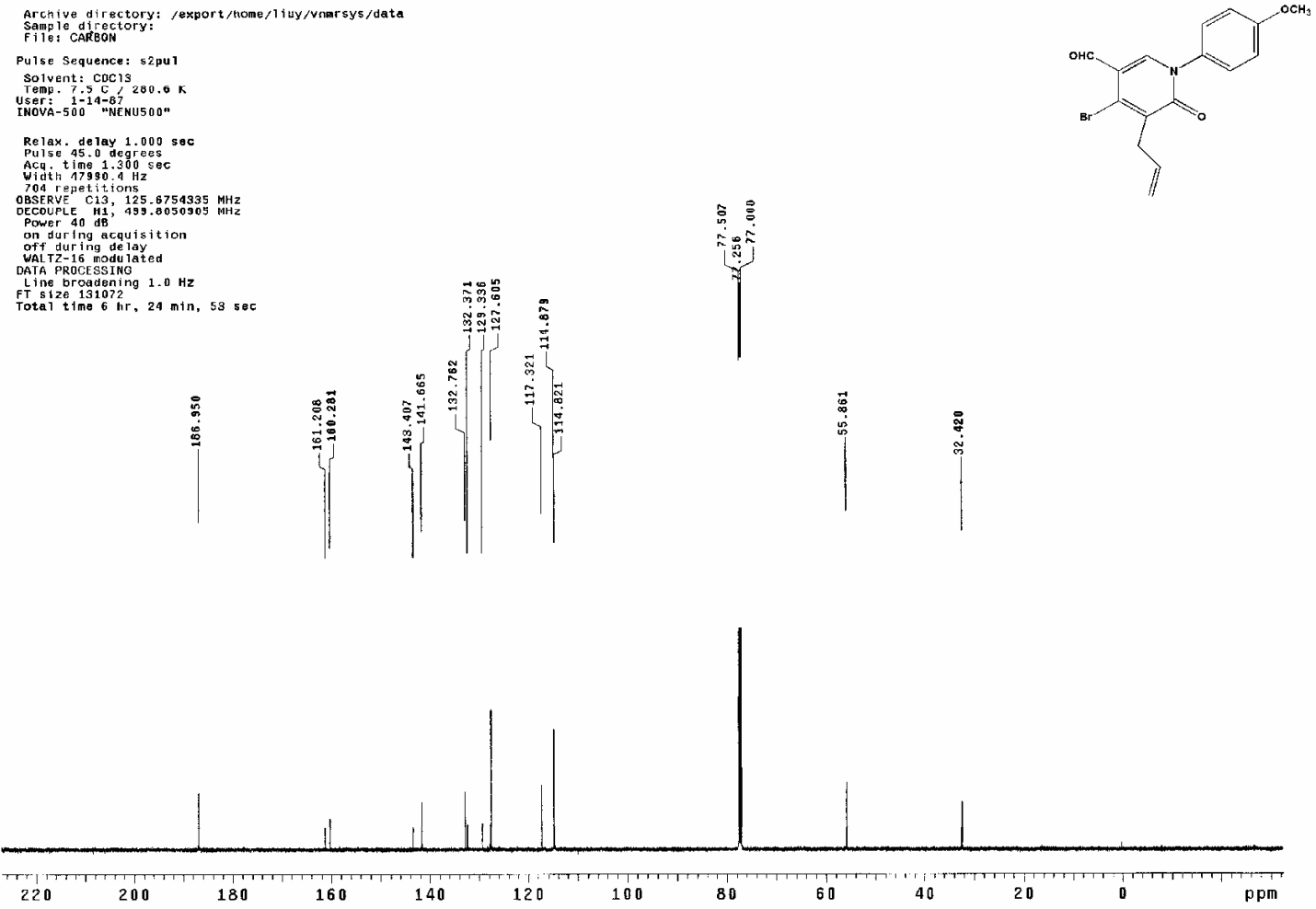
$2 \mathrm{~m} 1$

STANDARD PROTON PARAMETERS

Archive directory: /export/home/liuy/unar sys/dat
sample le directory:
file:

File: PROTOM

Pulse Sequence: s2pur

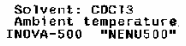

Regax. De lay 1.000 sec
Pulse 45.0 yegrees

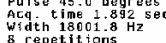

OBSERYE H1, 499.802587 M MHZ

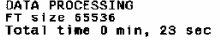
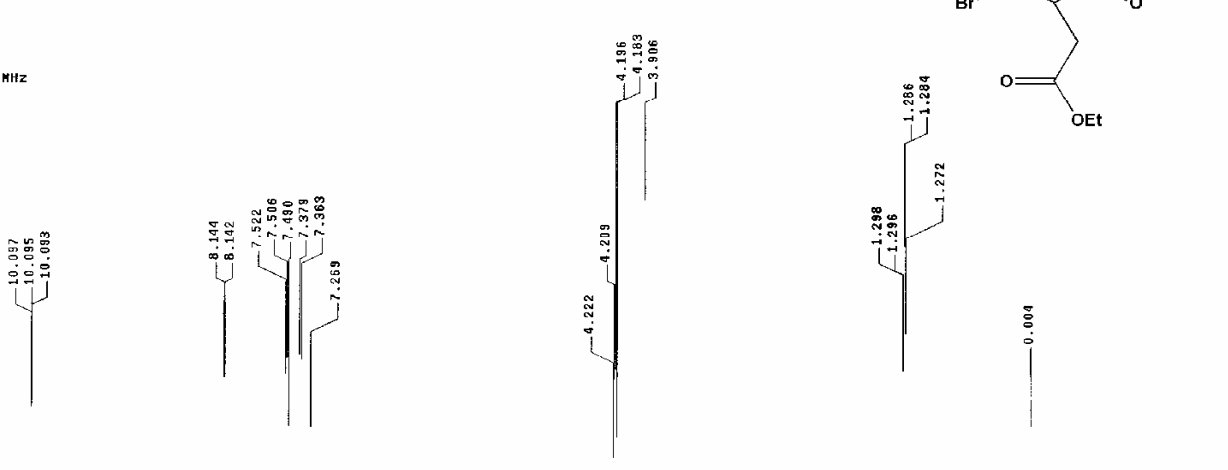

standaro carbon parameters

Archive directory: lexport/home/liuy/vinrsys/data
SAmple directory:
File: CARBON

Pulse sequence: $\mathrm{s} 2 \mathrm{pu}$

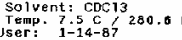
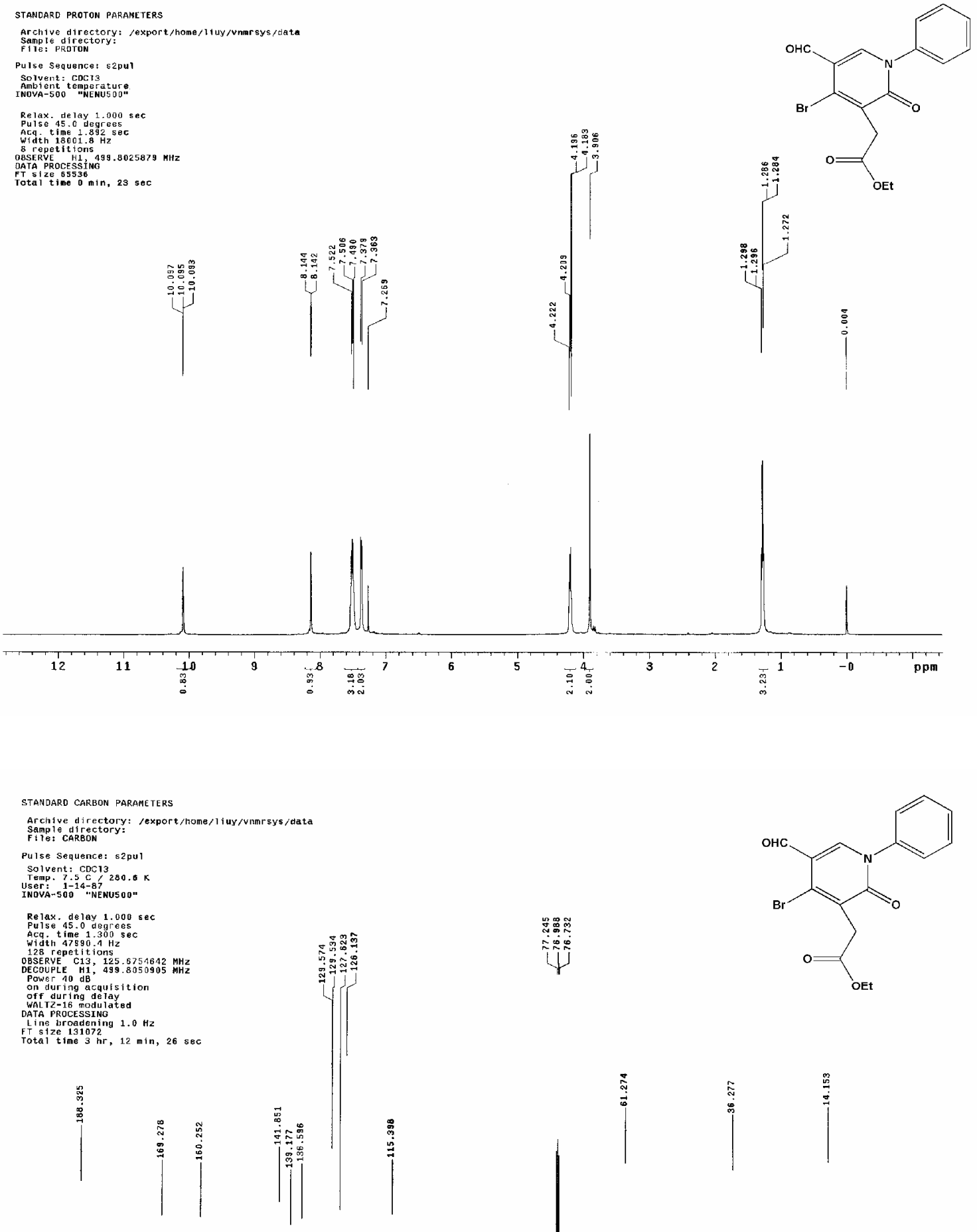

DATA Phroadening 1.0

Total time $3 \mathrm{hr}, 12$

10
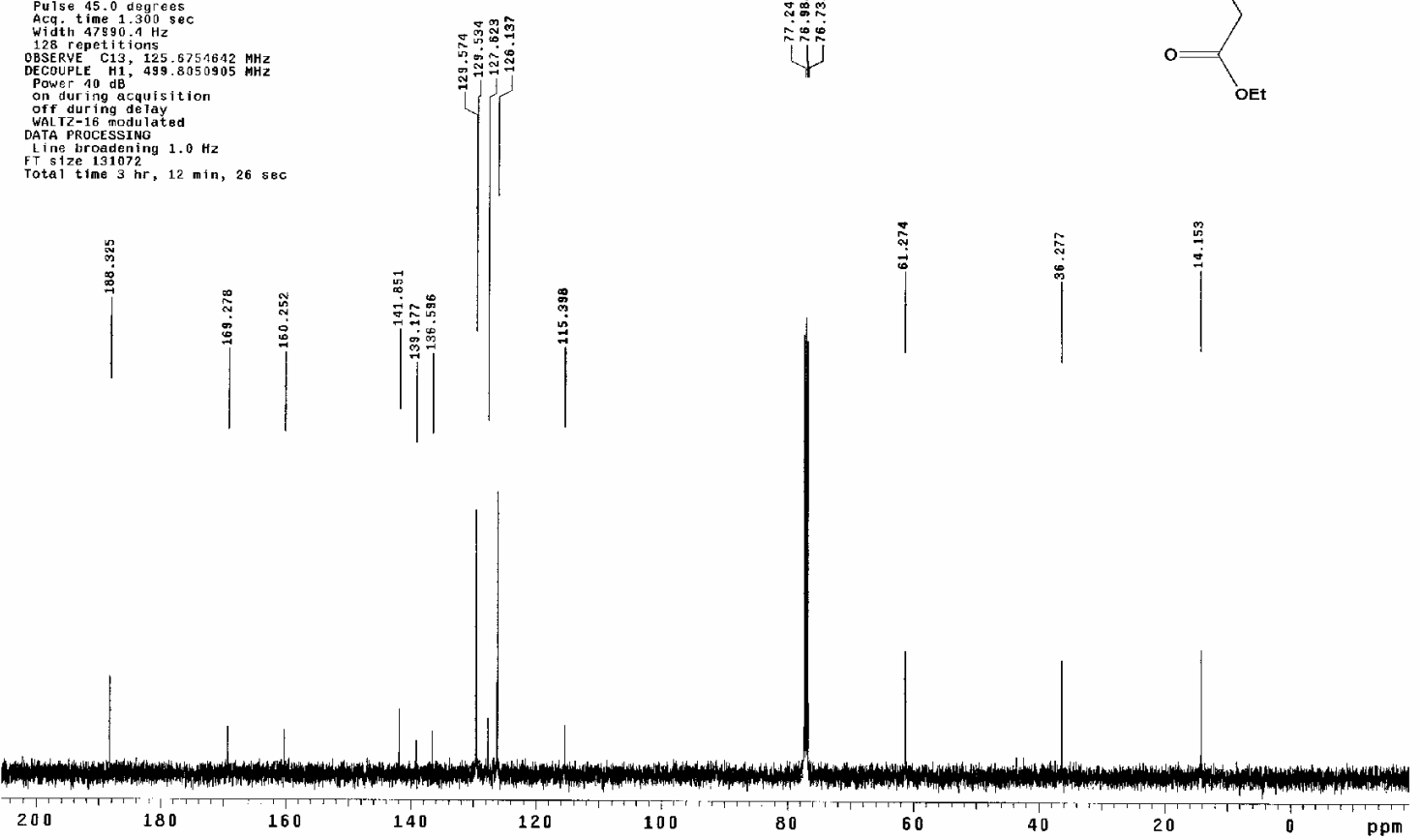
STANDARo PRot TN PARAMETERS

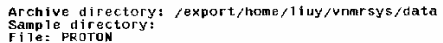

Pulse Sequence: s2pur

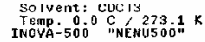

Re lax. defay 1,000 sec

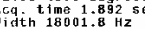

OBSERVE H1 $499.8025906 \mathrm{MHZ}$

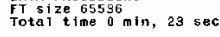
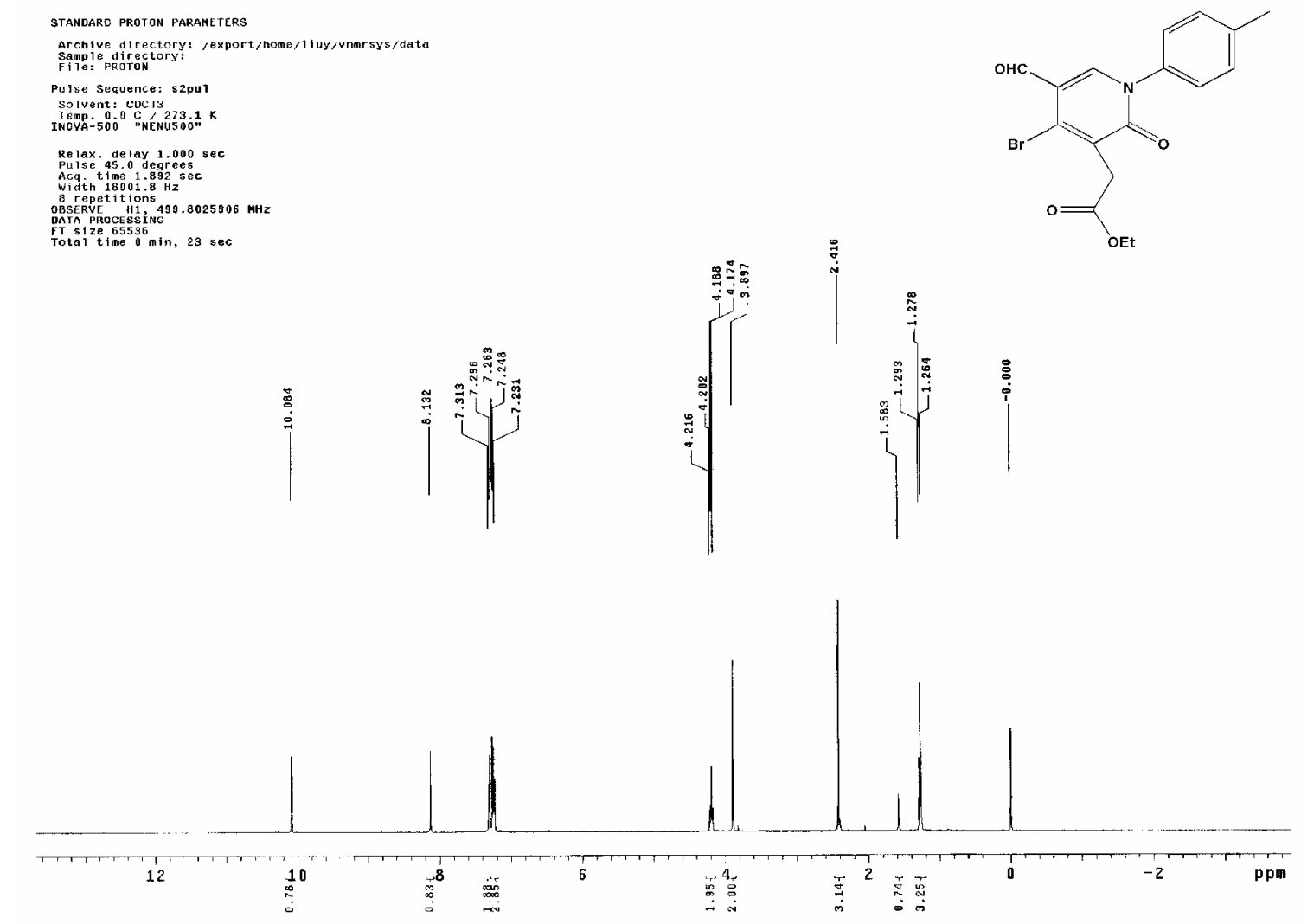

STANDARD CARBON PARAMETERS

Arehive directory: /export/home/liuy/vnnrsys/date

Sampie directory
file: CARBON

Pulse Sequence: 52pul

Solvent: CnC13 13

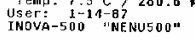

Re lax. de lay 1 - 000 sec
Pulse 45.0 degrees

Acu time $1.500 \mathrm{~s}$
widith $47990.1 \mathrm{~Hz}$

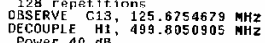

Power 40 ab
on during acquisition

off turing degy
WAT

Fit sije 131072
rotal time 6 hr, $24 \mathrm{~min}, 53 \mathrm{sec}$

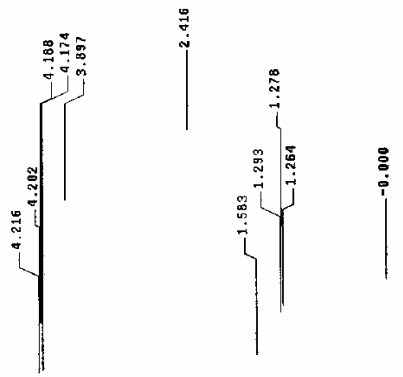

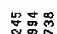

闪

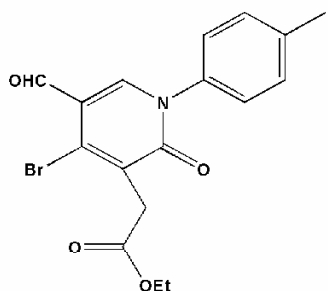

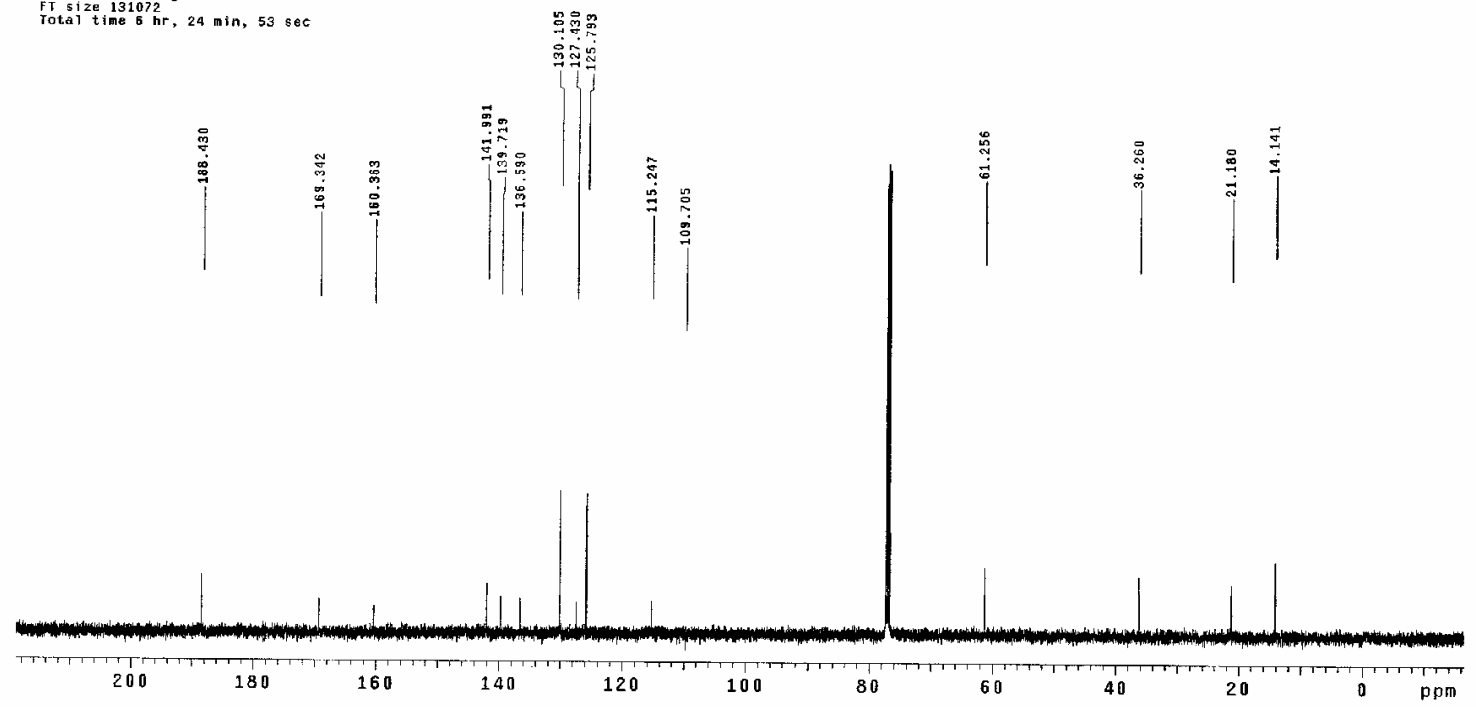


STAMIARO Proton PARAMETERS

Archive directory: export/mone/lluy/vnn sys/data

Puiser Sequence: $=5$ is

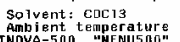

Relax. diblay $1.000 \mathrm{sec}$
pulse 45.0 degrees

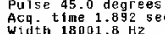

8 repetitions $\mathrm{Hz}$

DATA PROCESSING
TT Size 653636
Total time 0 min, $23 \mathrm{soc}$
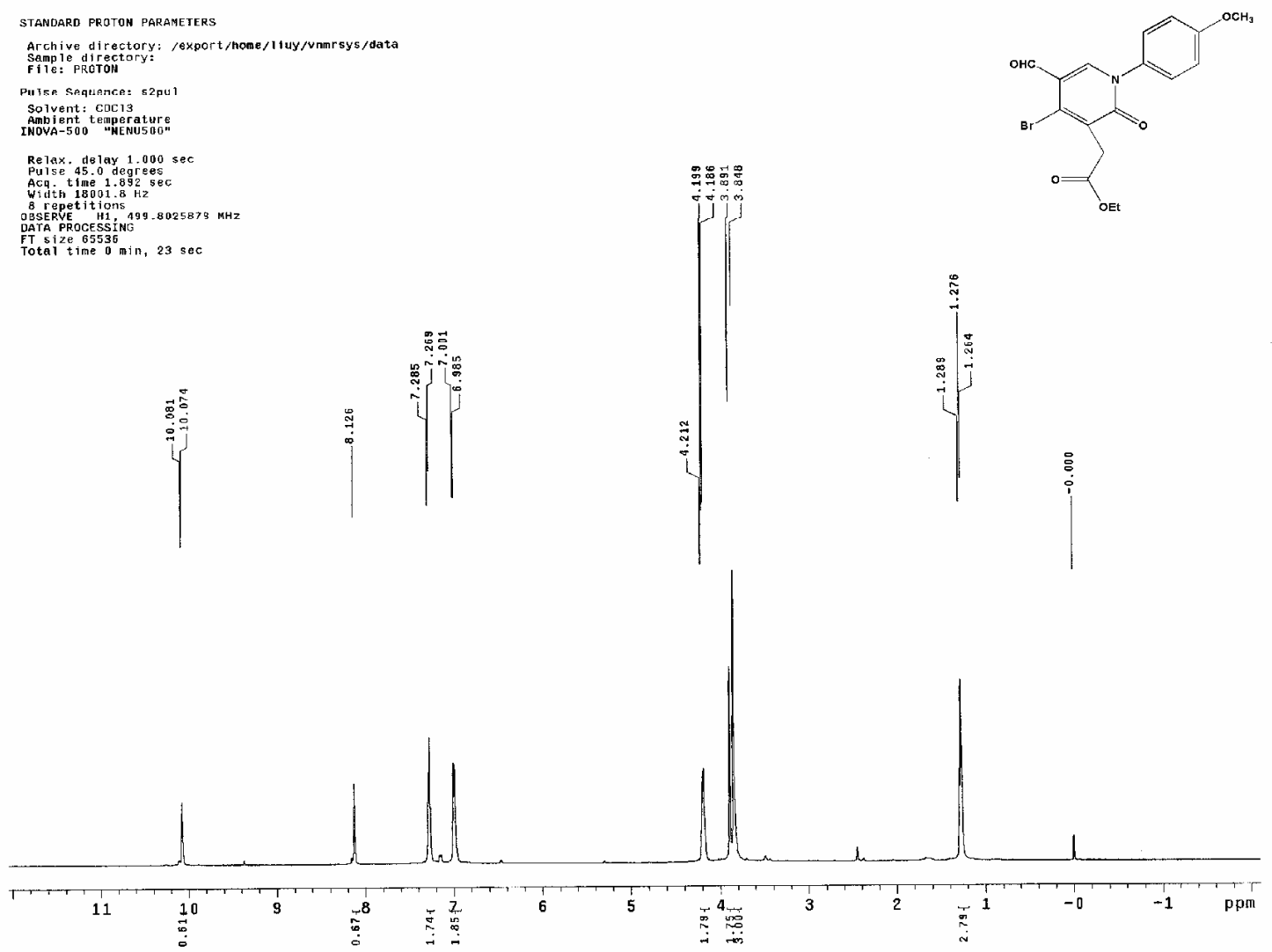

STANDARD CARBON PARAKETERS

Archive directory: /export/home/1luy/vmursys/data
Sanple directory:

Pule soguencos

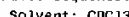

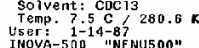

Relax. de lay $1.000 \mathrm{sec}$

Relax de lay 1.000 sec
Pulse 45 . 0 degrees
Acq. t time 1.300 sec

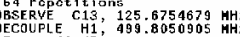

Power 40 dB' decisition

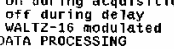

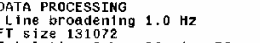
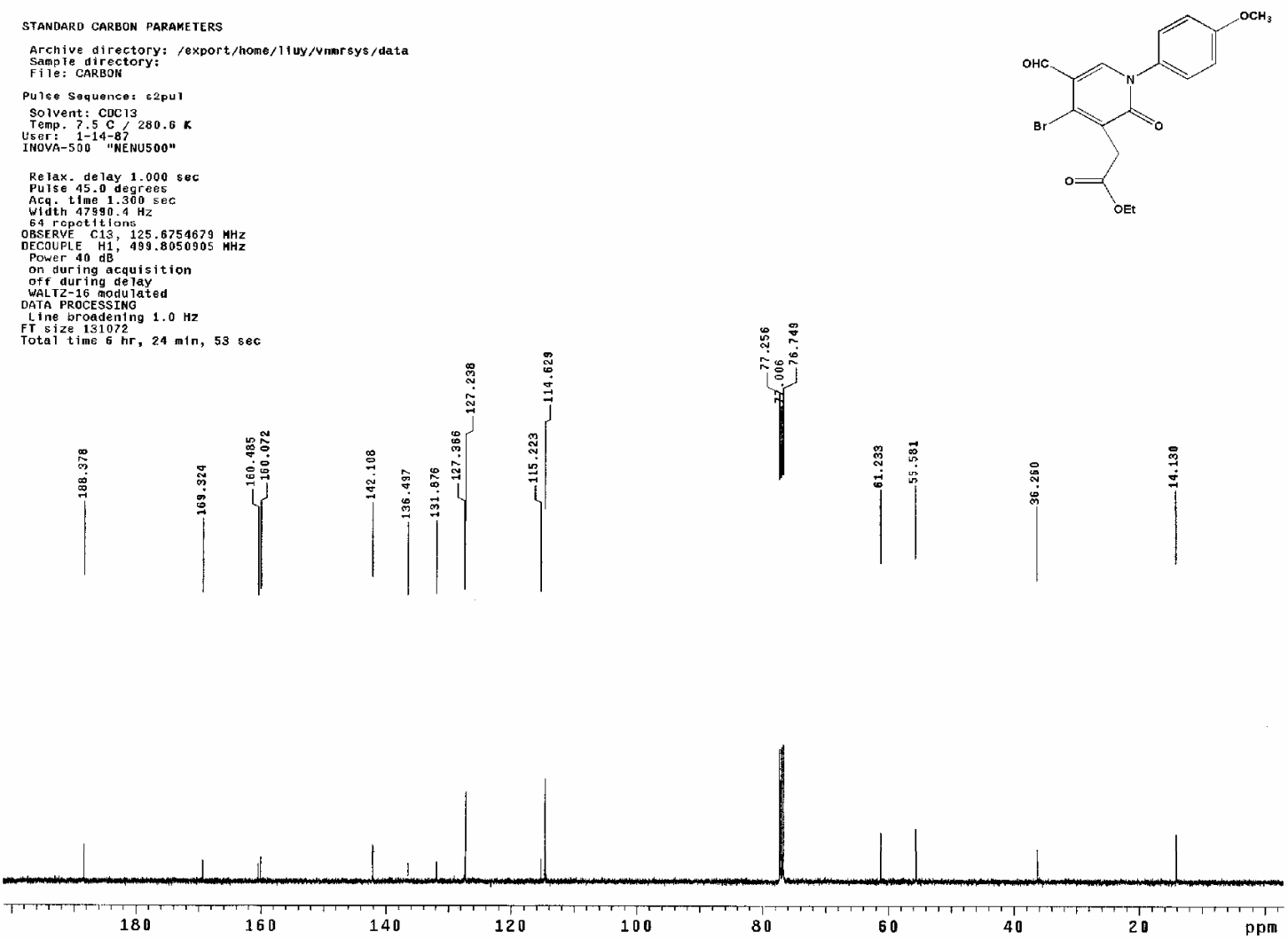
STANDARO PROTON PARAMETERS

Archive directory: /export/home/1 i uy/unmrsys/dat.

PuTse Sequence: S2pui

Solvent: CDC13 $280.6 \mathrm{~K}$

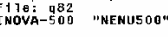

Relax. delay 1.000 sec

Acy. time 1.892

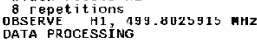

Total time 0 min, 23 sec
Tot
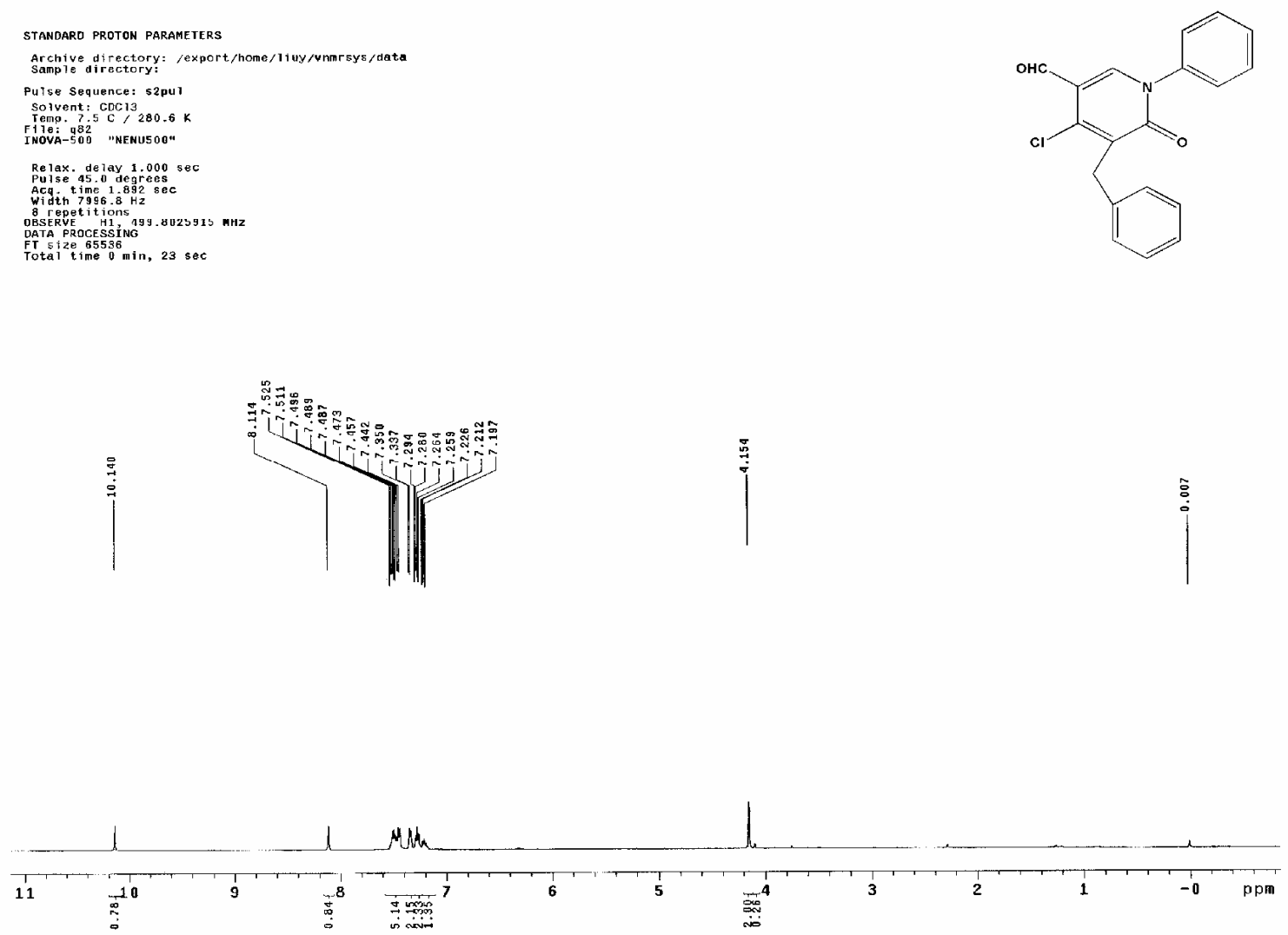

STANDARD CARBON PARAMETERS

Archive directory: /export/home/ i i iy/vimrsys/data
Sample dir ectory:

Pulse Sequence: 520

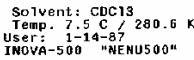

Relax. de lay $1,000 \mathrm{sec}$
Pulse 45.0 degrees

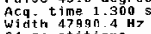

OSERE C18, 125.6754327 HHz
OECOUPLE H1, 499.8050905 HHZ

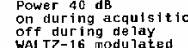

WALTZ-16 modulated
DATA PROCESSTNG

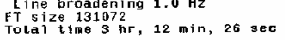
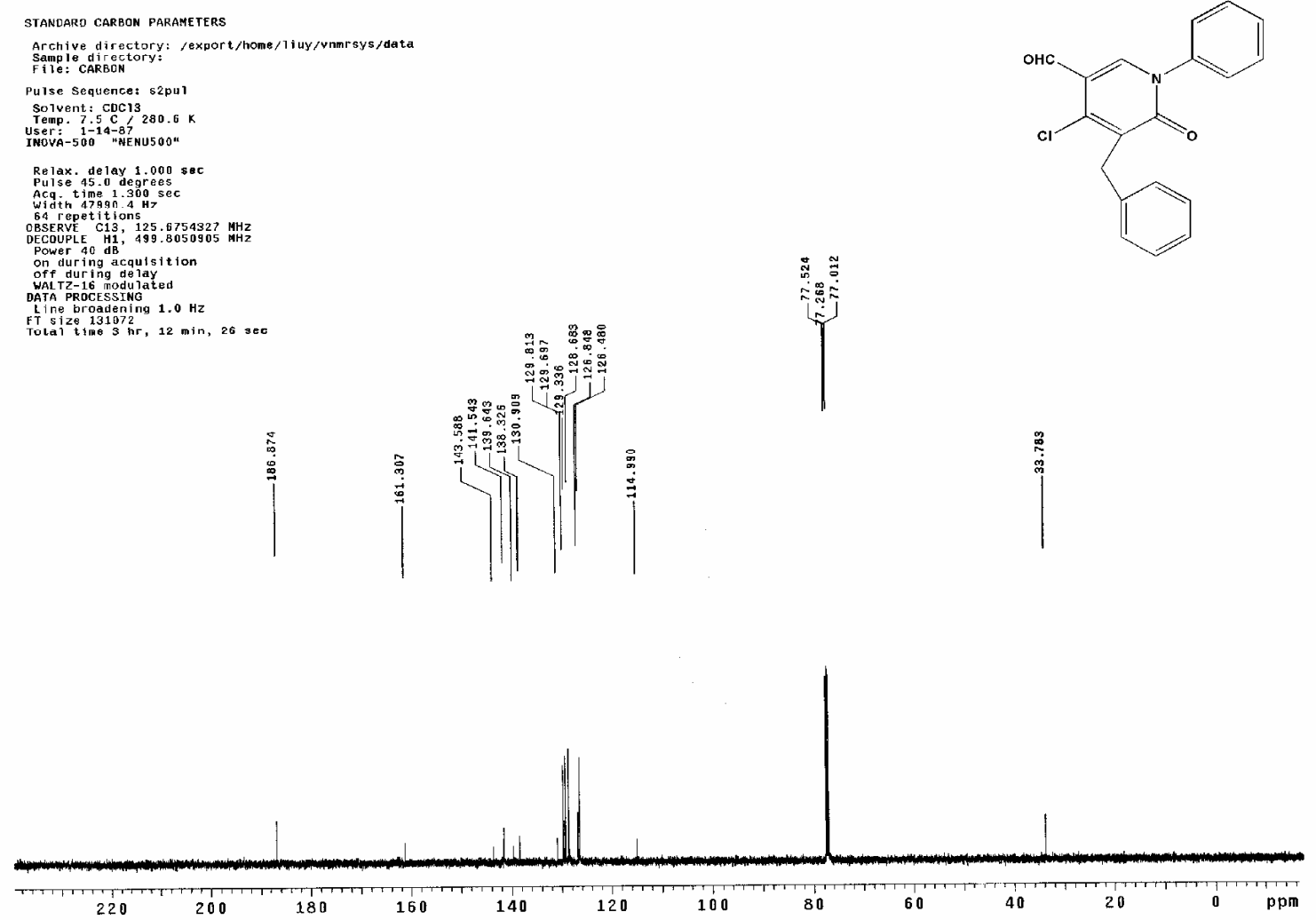
standard proton parameters

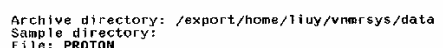

File: PROTON

Pulse Sequence: s2put
Solvent: $\operatorname{coc} 13$

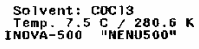

Rolax. de lay $1.000 \mathrm{sec}$
Pulse 45.0 degrees
Acd $t$ time 1.392 sec

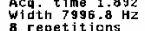

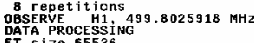

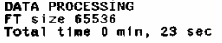
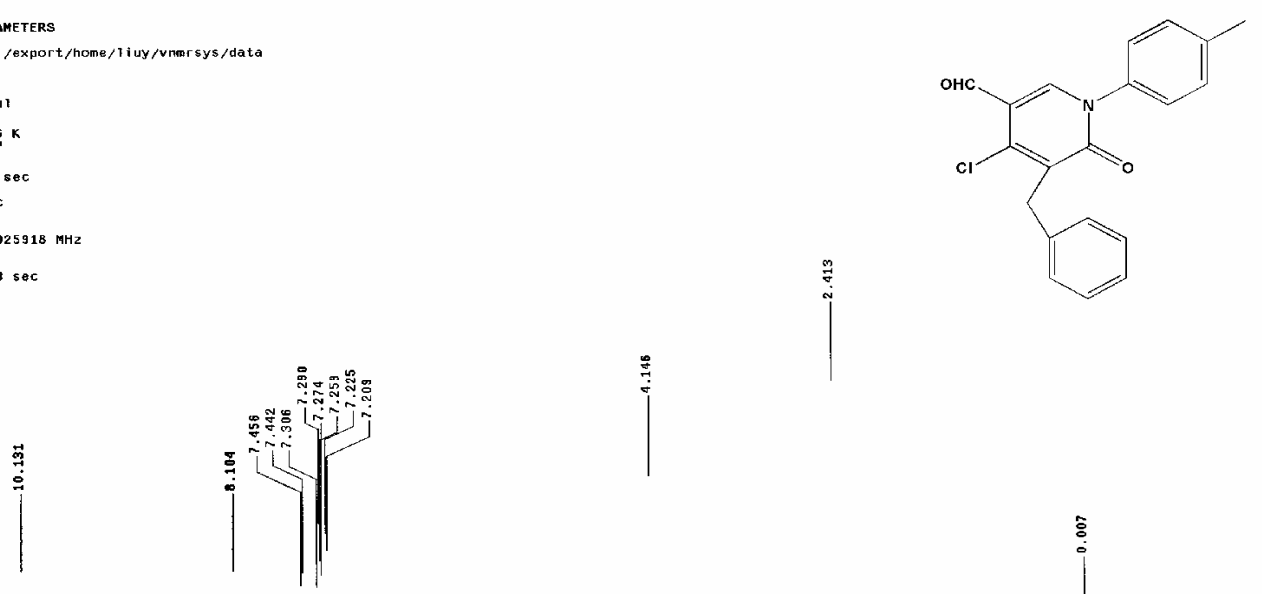

$\mid$

|

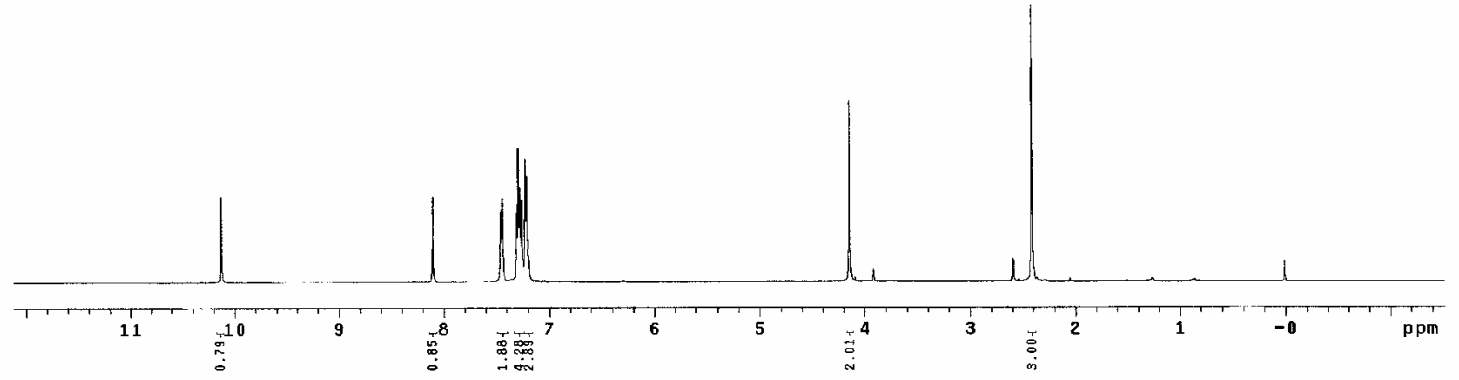

STANDARO GaRbON PARAMETERS

Archlve di rectory: lexport/home/liuy/vrarsys/data
Sample directtory:
File: CARBON

Pulse Sequence: $52 \mathrm{pul}$

Solvent: $\mathrm{CDCl}^{2} 3 \mathrm{~s}, \mathrm{~K}$

USer: $1-14-8$ ?

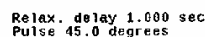

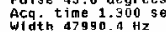

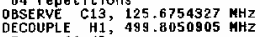

Power ao ab
on during acquisition

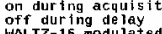

DATA PROCESING
Line broadening $1.0 \mathrm{~Hz}$
FT size 131072

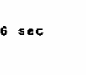

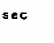
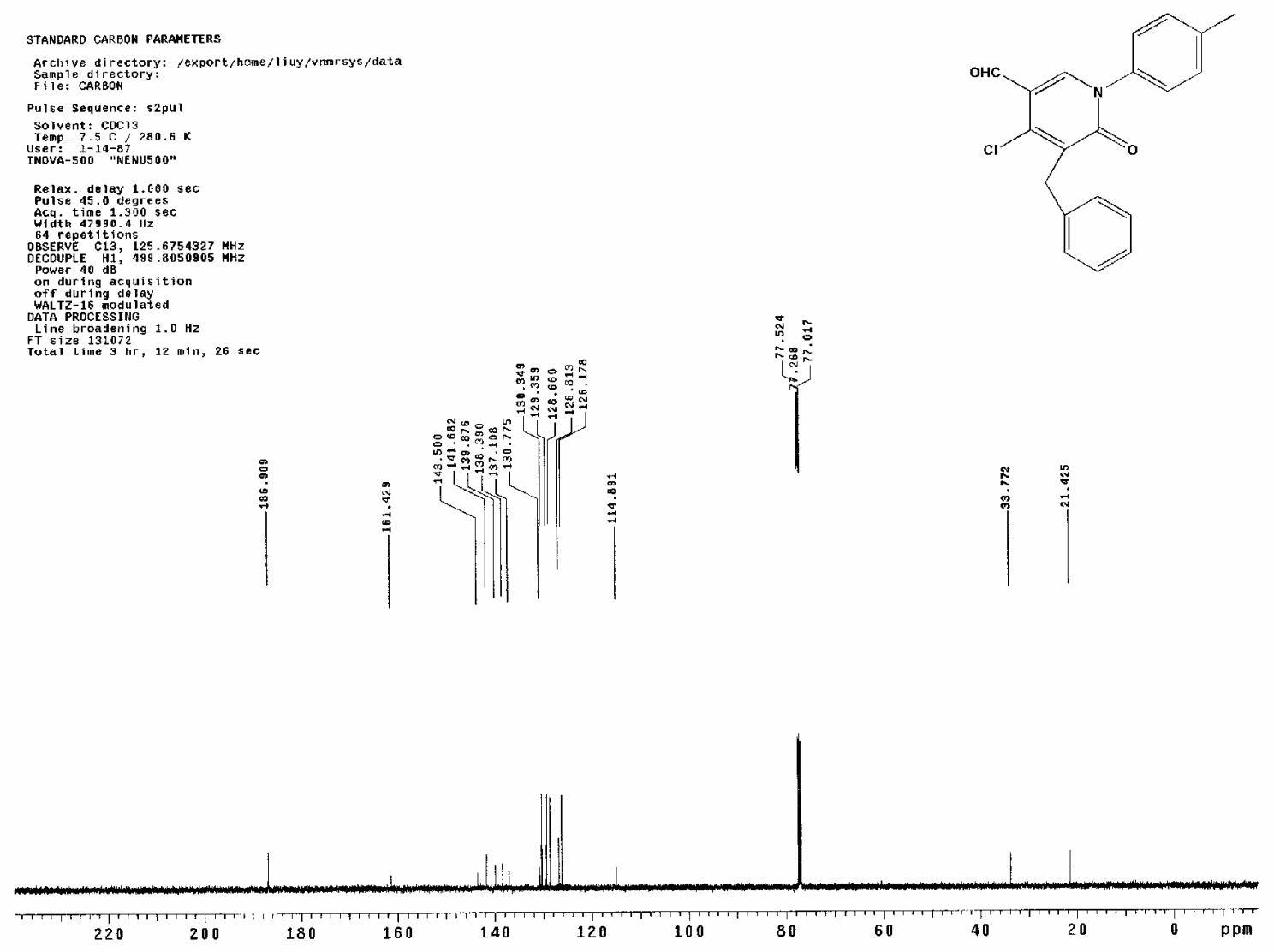
STANDARD PROTON PARAMETERS

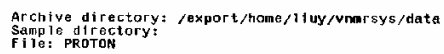

Pulse Sequence: s2pur

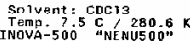

Relax. delay 1.000 sec
Pulsc 45.0 degress

Acqu ${ }^{t}{ }^{2}$ me $1.892 \mathrm{sec}$

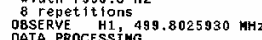

OATA PROCESSTHG
FT Size 6536 .
Total time 0 min, $23 \mathrm{sec}$
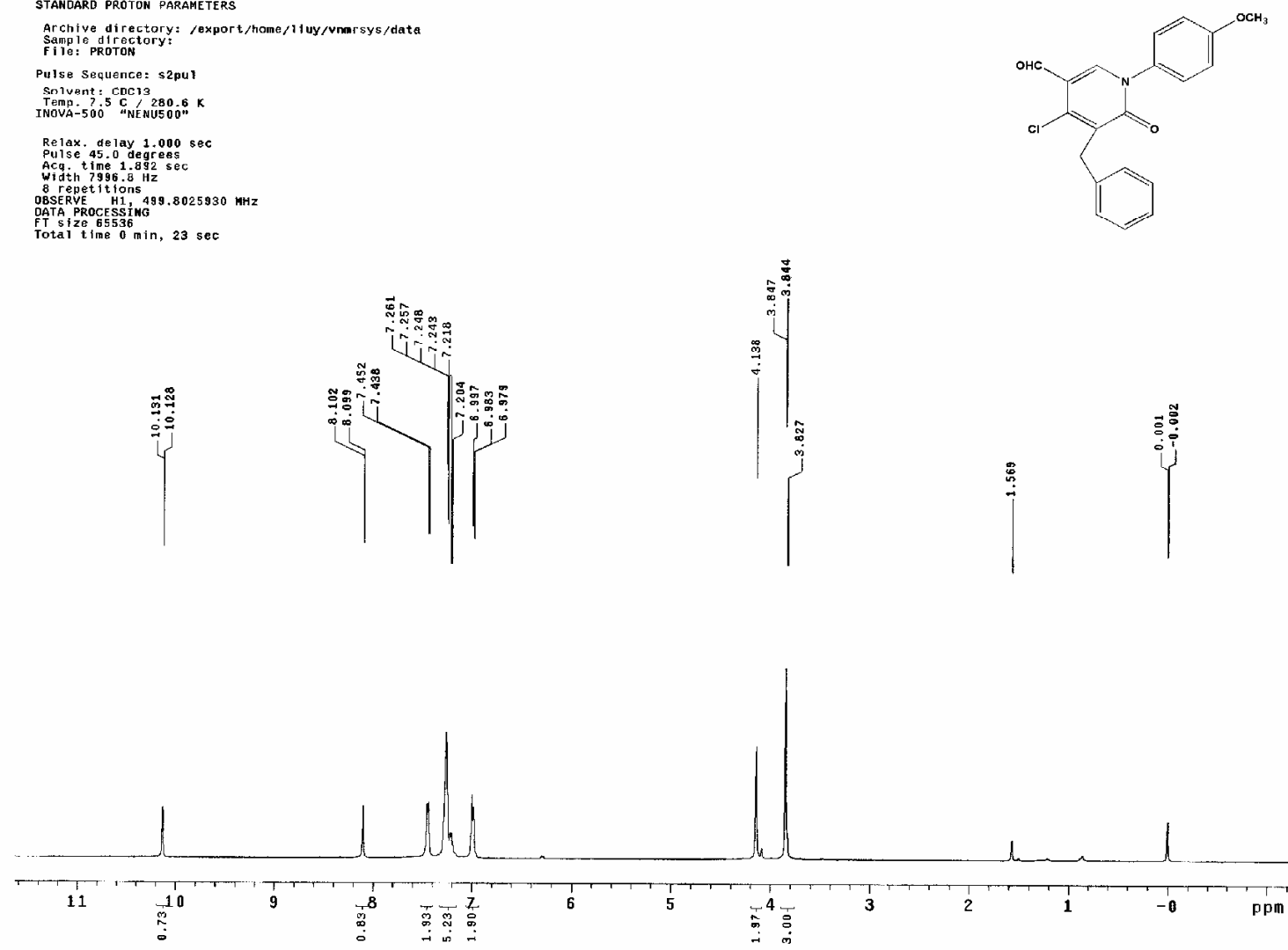

STANUARD GARBon PARAMETER

Archive directory: /export/home/14uy/vnmrsys/data

Pulse sequence

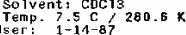

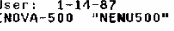

Relax. delay $1.000 \mathrm{sec}$
Puise 45.0 diegrees

Acg time 1.300 se

OBSERE C13, 125.6754708 MHZ
OECOUPLE H1, 499.8050905 MHZ

Power $90 \mathrm{~dB}$.

Off tur

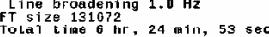
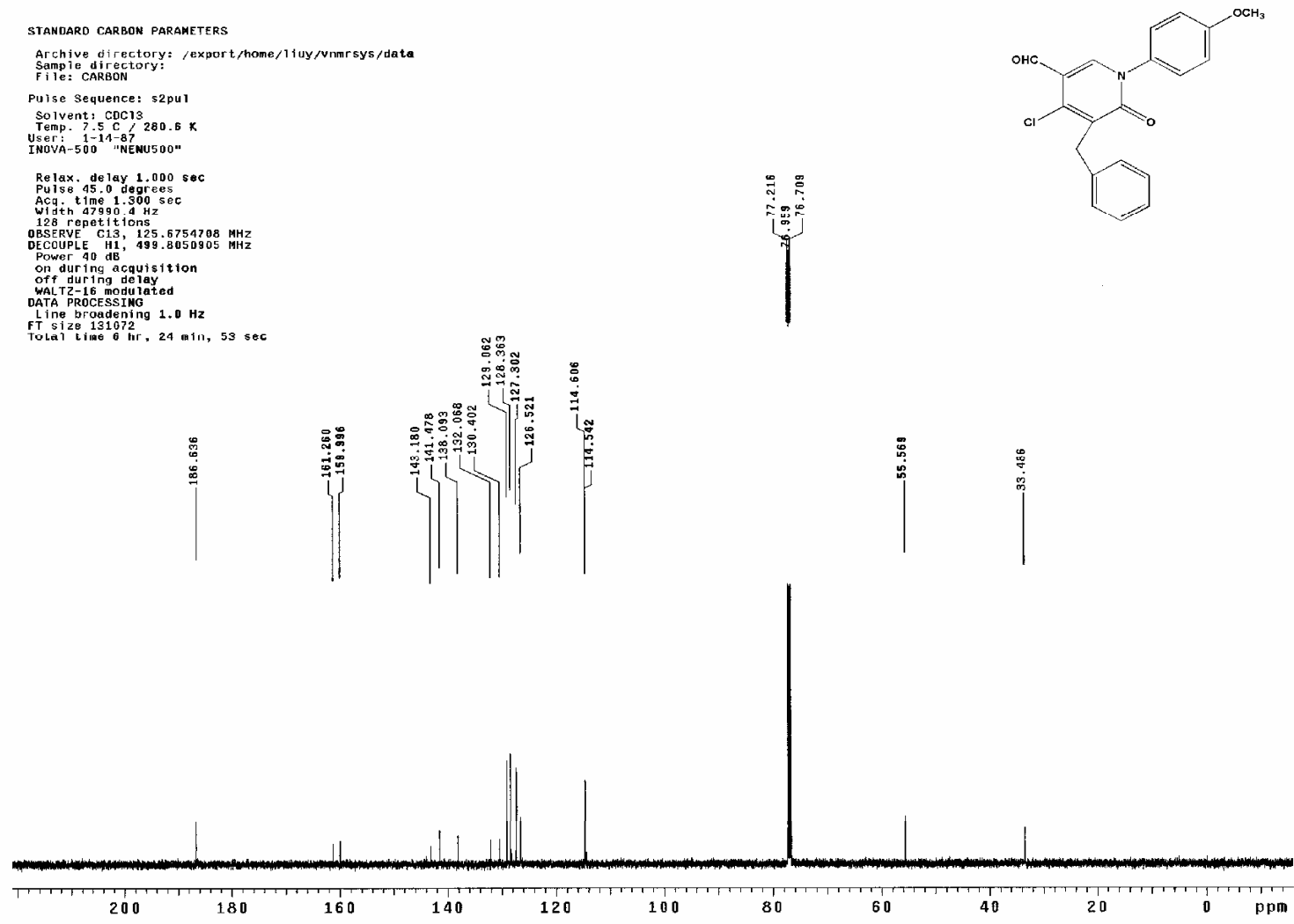
STANDARO PROTON PARAMETERS

Archive directory: lexport/home/liuy/unmrsys/data
Sarnple directory:

Finc: proton

Pulse Sequence: s2pul

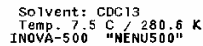

Rejax. delay $1.000 \mathrm{sec}$
Pulse 45.0 degrees

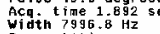

OESRYE H1 499.8025892 HHZ

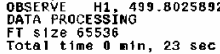
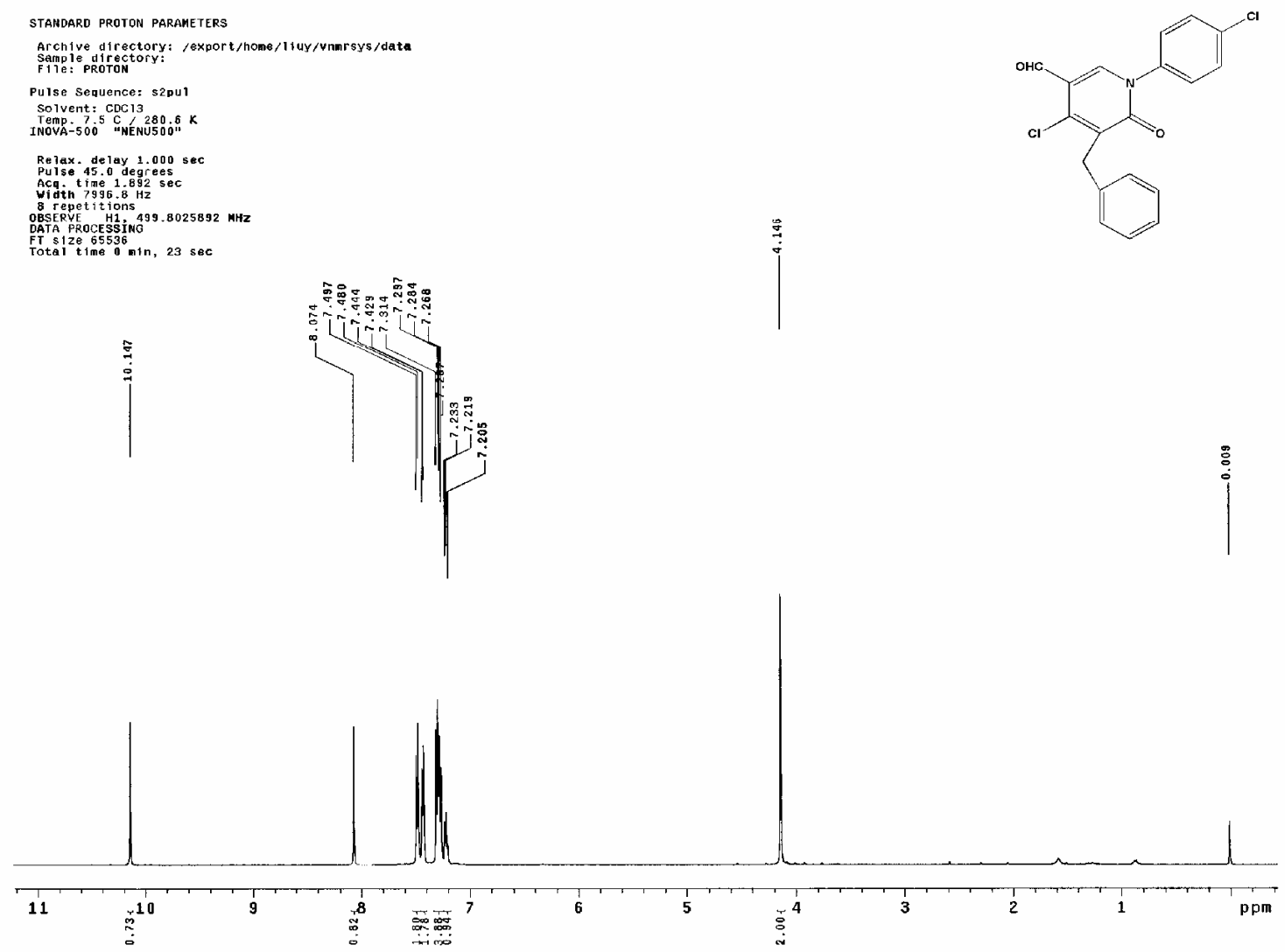

STANOARD GARBol PARAmeters

Archive directory: /export/home/liuy/vnmrsys/data

CARBON

Purse Sequence: s2pul

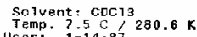

OSER: $1-14-87$
INOVA 500 "NENUS 00"

Re ax. de lay 1.000 se

Acg. time $1.300 \mathrm{sec}$

128 repetitions
OESERE C13, 125.6754335 HHZ
OECCUPLE H1, 499.8050905 HHZ

Power 40 dB
on during acquisition
off

off ururing de ay

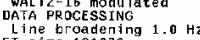

Line broadening $1.0 \mathrm{~Hz}$
FT size 131072
rotal time $6 \mathrm{hr}, 24 \mathrm{~min}, 53 \mathrm{sec}$

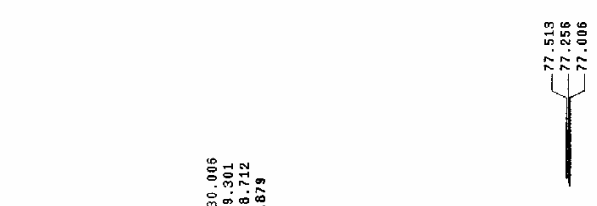

mo응

4
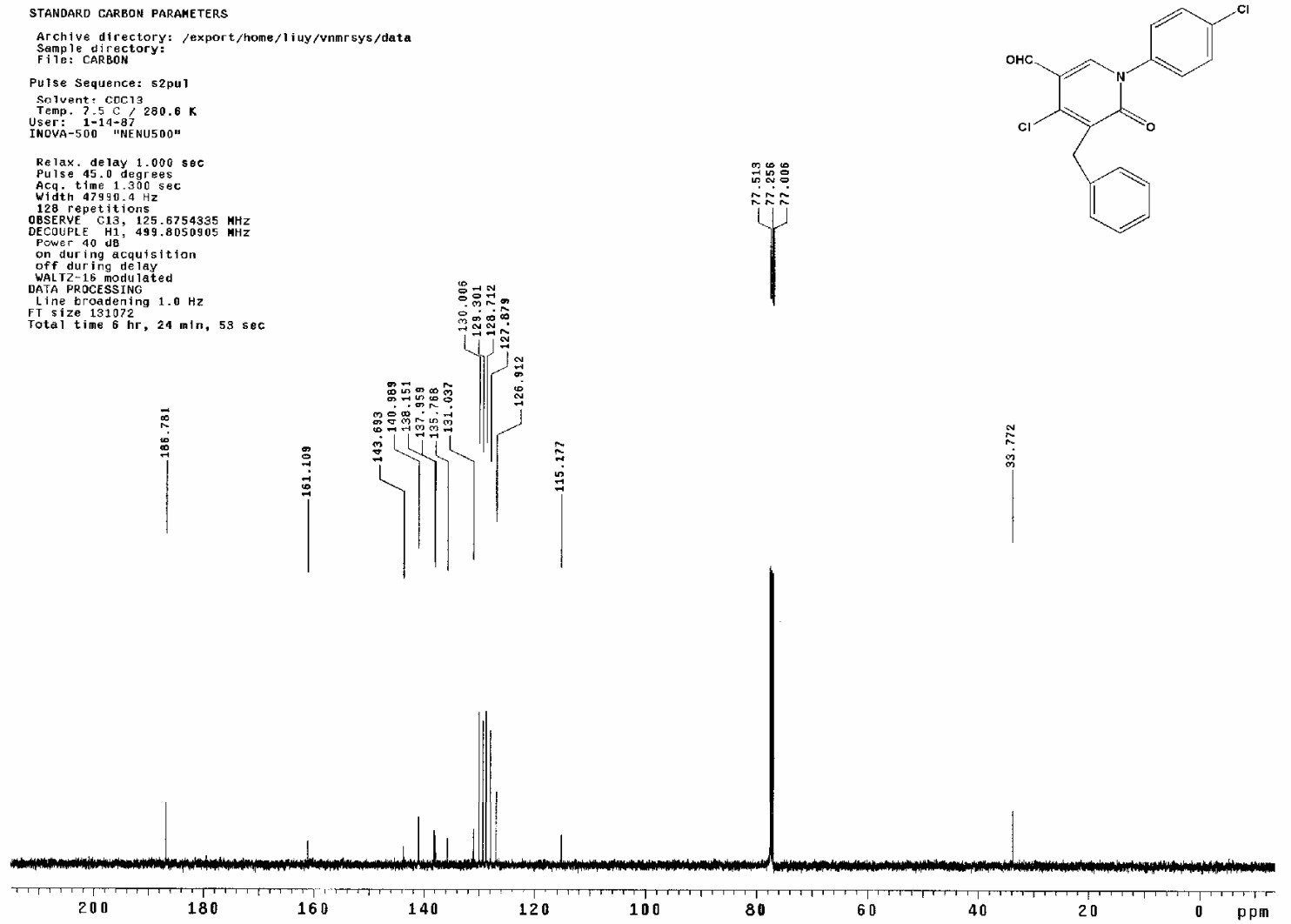
STANDARD PROTON PARAMETERS

Archive dir ectory: export/hame/li uy/unmrsys/data
Sample directory:
File: PROTout

Pu 1 se Sequence: s2pur

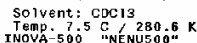

Relax. de 1ay $1.000 \mathrm{sec}$

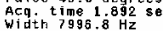

S rept it ions
OBSERVE H1, $499.8025915 \mathrm{MHZ}$

OBSERVE H1 A9 9.8025915
DATA
OATROESSING

FT size. 65536
Total time 0 min, 23 sec
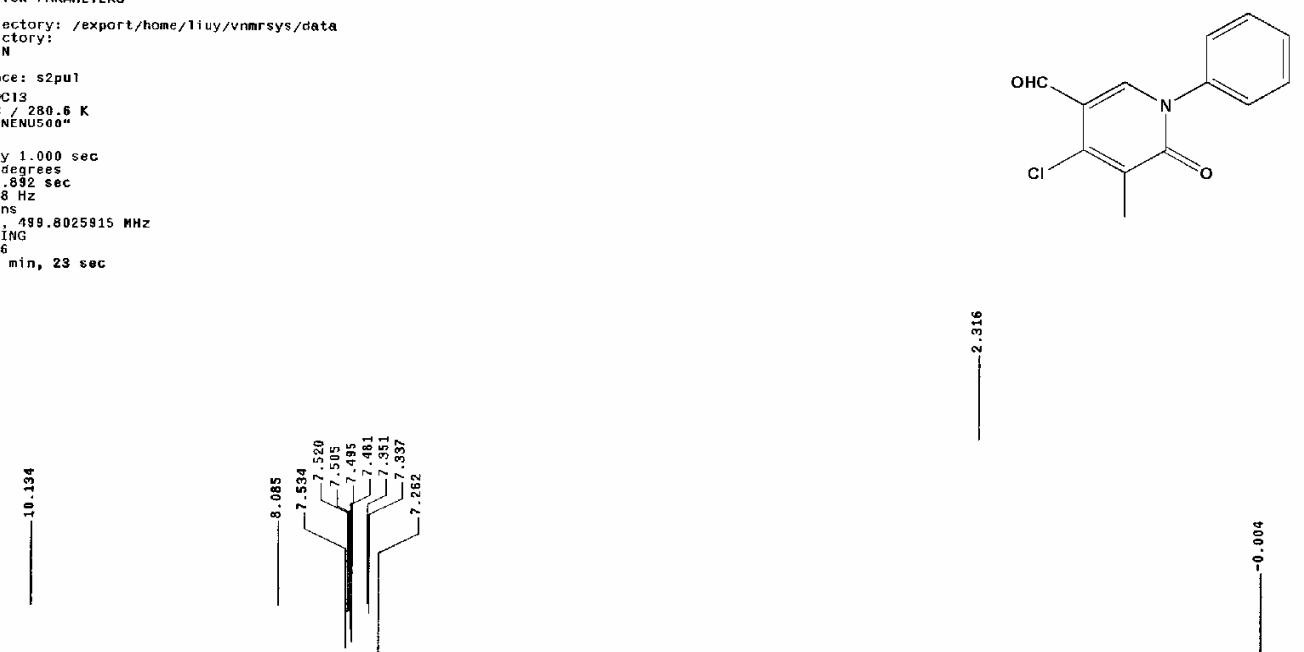

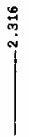

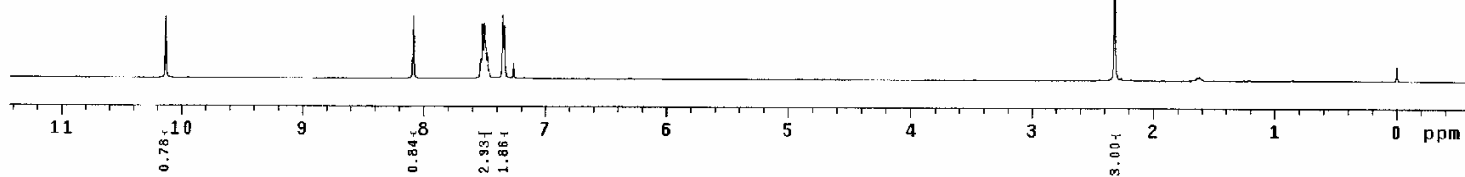

Tandard carbon parameters

Archive di rectory: /export/home/liuy/vnmrsys/data

Pulse Sequence: $52 \mathrm{pu}$

Solvent: $\operatorname{coc} 13$

User: 1-114-8? 280.6
ENOVA-500 "NENUS0a"

Relax. de lay 1.000500
Pulse 45.0 derees

Acq. time 1.300 sec

idth 47990.4 h

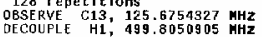

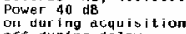

off during de lay

line broadening $1.0 \mathrm{~Hz}$

Iotal time $6 \mathrm{hr}, 24$ min, 53 soc
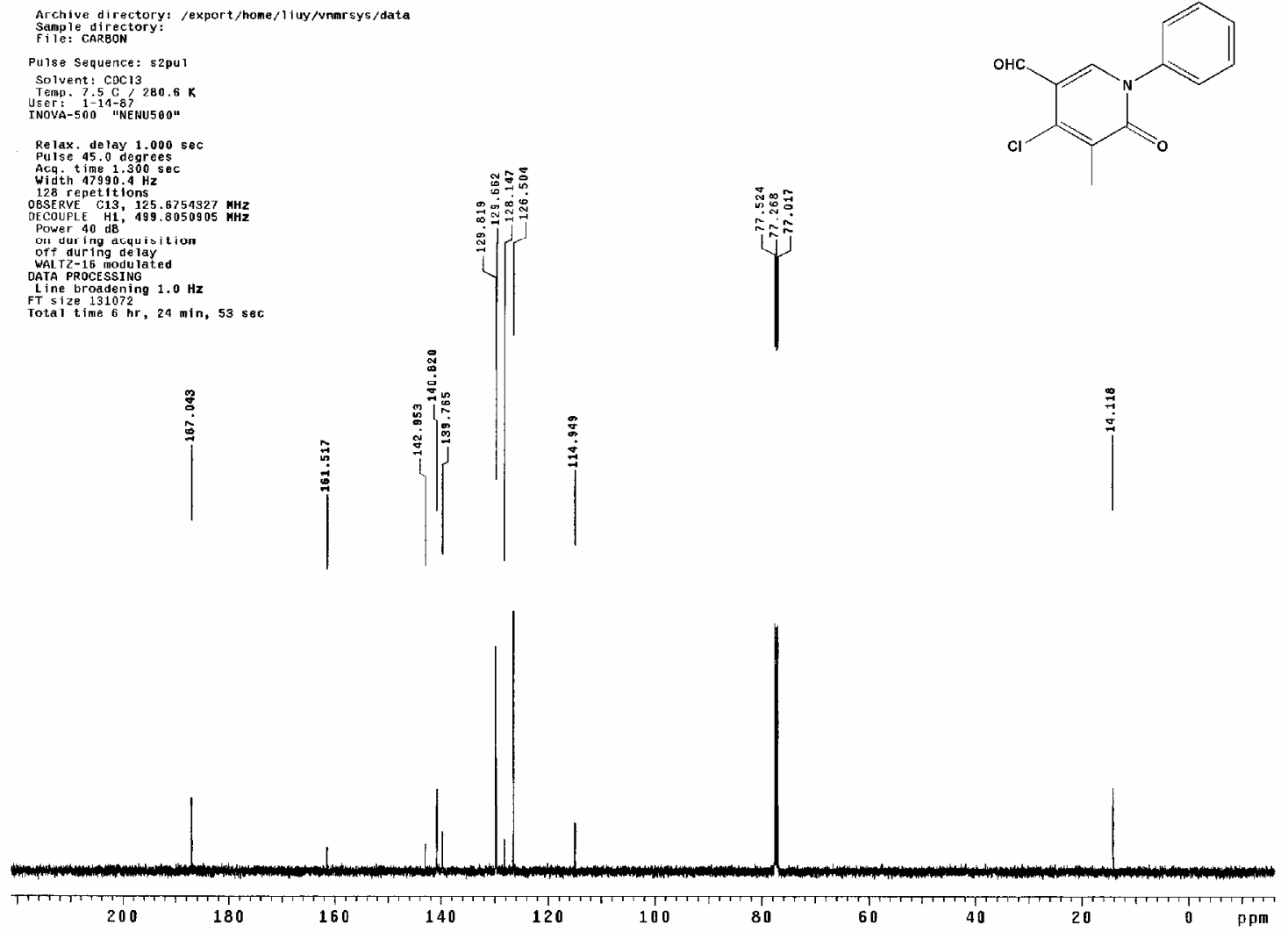
$2 \mathrm{p} 2$

Standarb proton papameters

Arehive directory: /export/home/liu/ynmesys/data
Sample direct tory:

Pulse sequence:

Solvent: $\operatorname{CDC} 13$

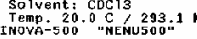

Relax. de lay 1.000 sec
Pulse 45.0 denrees

Act time 1.892 sec
width $11999.4 \mathrm{~Hz}$

S repetitions
OBSERE
OBSE

Total time $0 \mathrm{~min}, 23 \mathrm{sec}$
TT
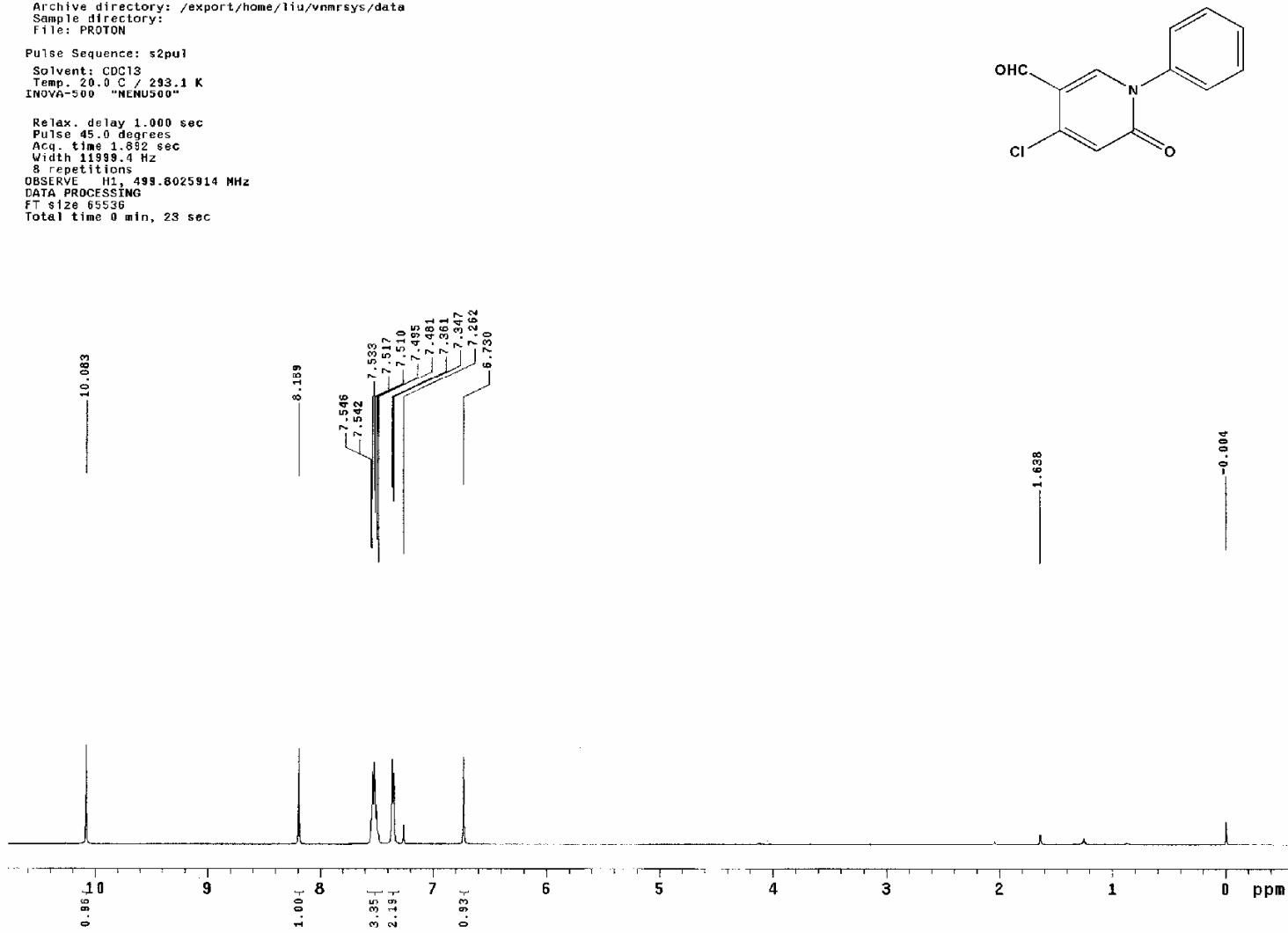

STANDARD GARBON PARAMETERS

Archlue di rectary: lexport/home/liuy/vnmrsys/data
Sample directory:

Pron

Pulse Sequence: \$2pul

Solvent: $\operatorname{CDC} 13$ s20

USer: i-19-87 280.6

Re lax. de lay 1.000 sec
Pulse 45.0 degrees

Aca time $1.300 \mathrm{sec}$

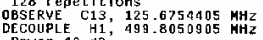

Power 40 de
on dur ing acquistion
off during

YALTZ-16 modulated

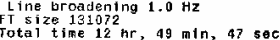

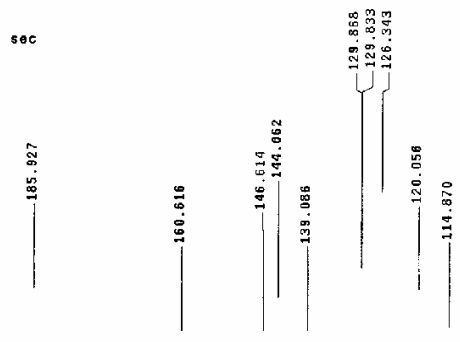

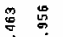
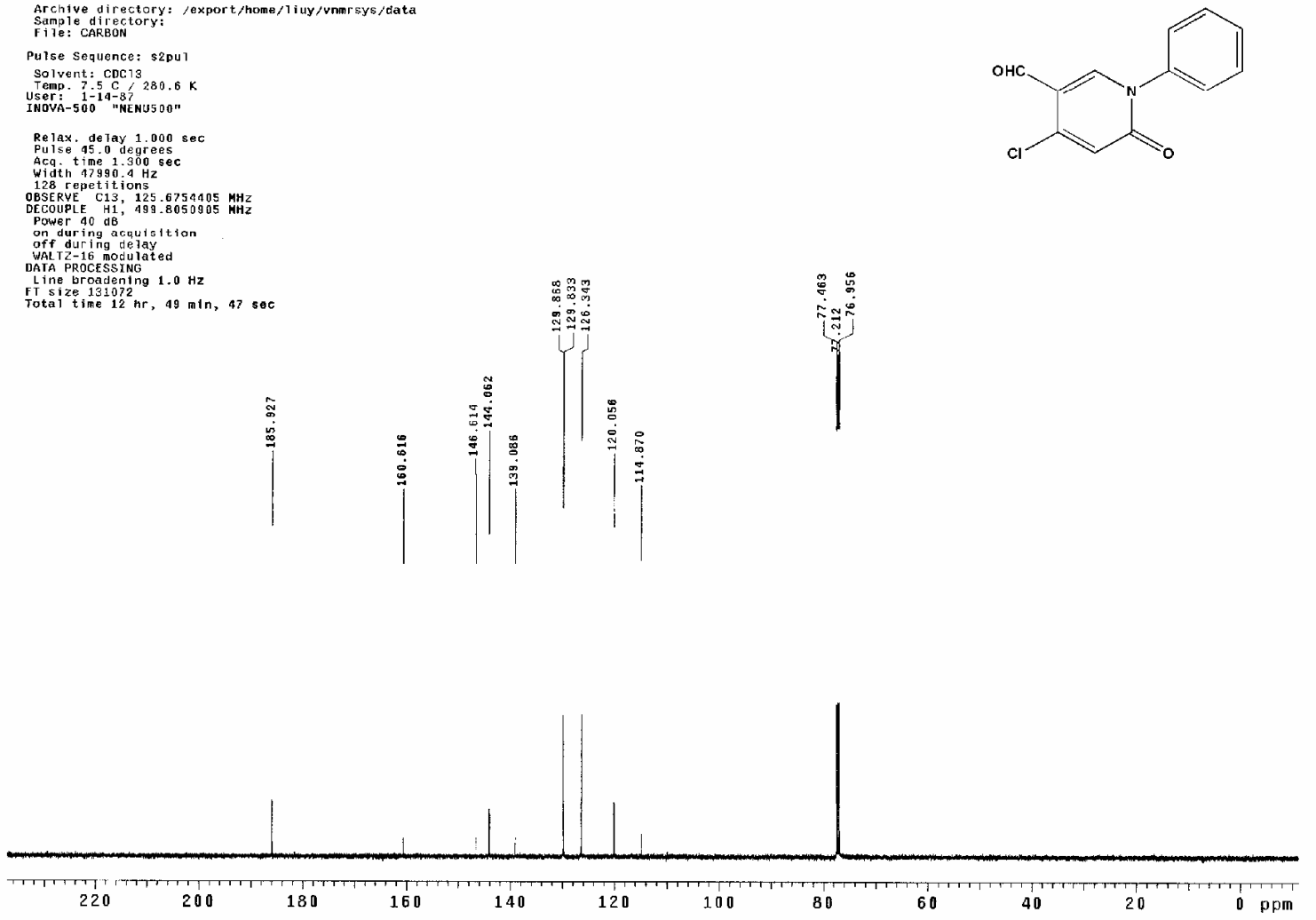
SIANDARD PRDTON PARAMETERS

Archive directory: /expart/home/Ituy/vnmrsys/data

File: PROT

Pulse Sequence: s2pur

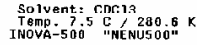

Relax delay 1,000 sec
Pulse 45.0 degres

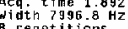

8 reptitions
OBSERYV

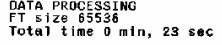
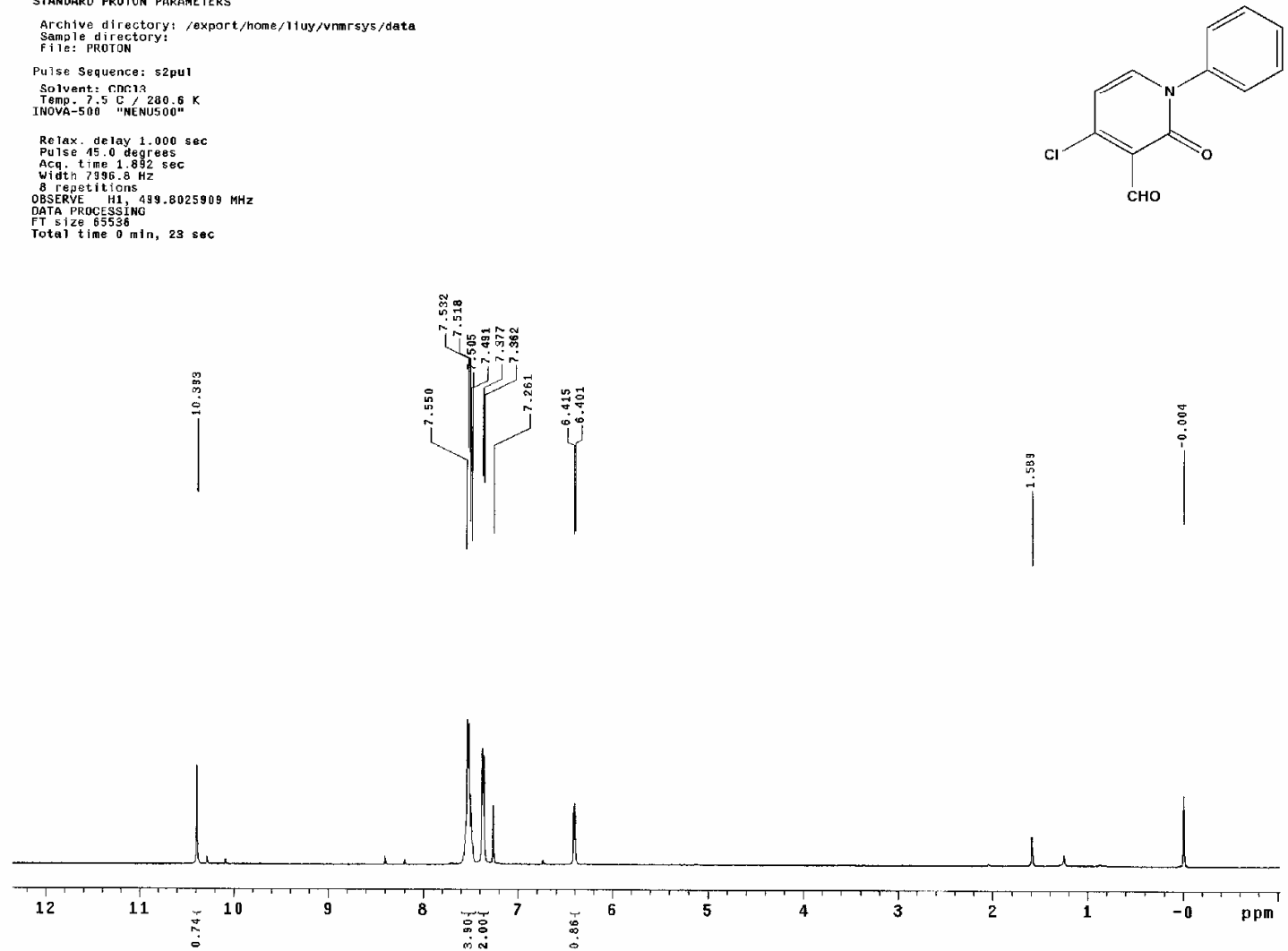

StANDARD CARbon PARAmeTER

Archive directory: /export/home/liuy/vnmrsys/data

i le: CARBON

Pulse Saquence: 52put

Solvent: Coct 13
Temp. 7.5 C 13 . 280.6

SMOVA-500 "NERUS T0"

Relax. delay $1.000 \mathrm{sec}$
Puise 45.0 degrees

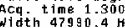

S
BSERVE C13, 125.6754679 MHZ
OECOUPLE H1, 499.8050905 MHZ

Power 40 de
on dur ing acquisition
off during delay

off during telay
waAL L

DATA PROCESSING
Iine brodeding $1.0 \mathrm{~Hz}$

Tine broadening $1.0 \mathrm{~Hz}$
TT size $1310 \mathrm{~g} 2$
Total time $6 \mathrm{hr}, 24 \mathrm{~min}, 53 \mathrm{sec}$
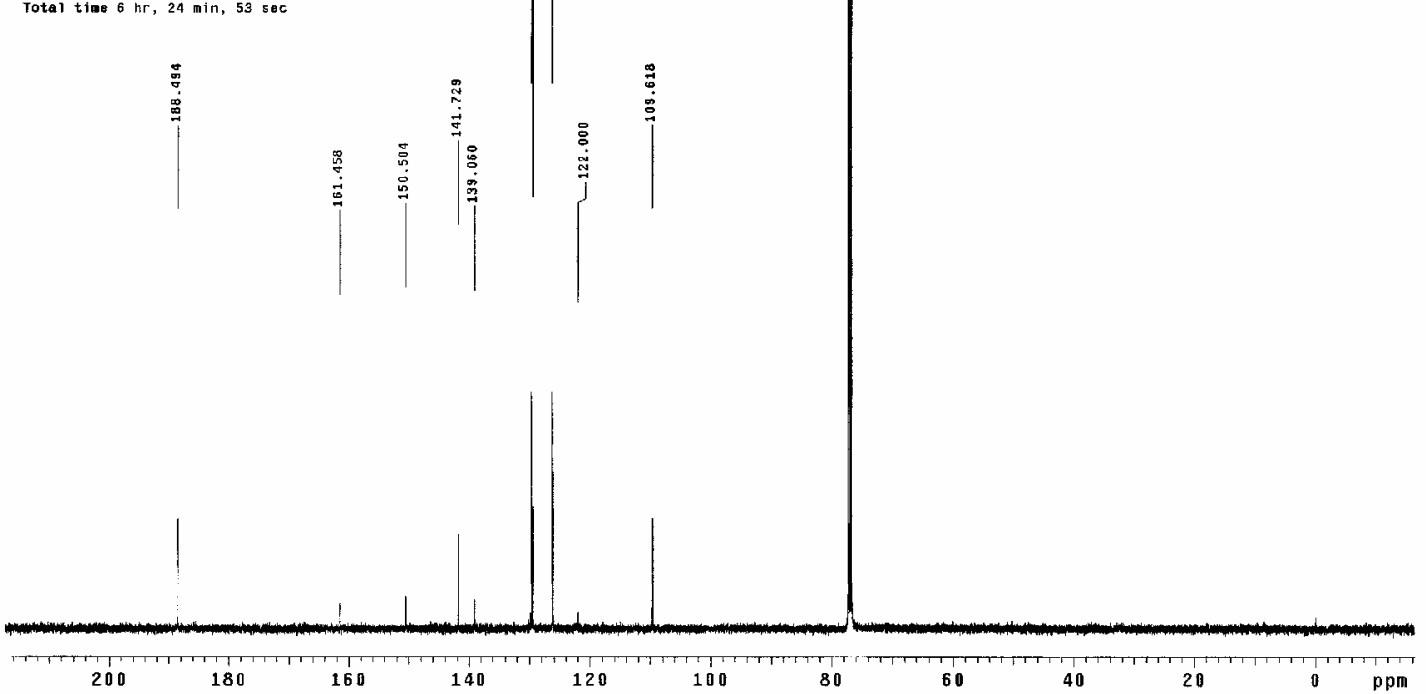


\section{$2 q 2$}

STANDARg PRGTON PARAMETERS

Archive directory: /export/home/i ity/unnrsys/data
Sample directory:
file: PROTOH

Pulse Sequence: s2pul

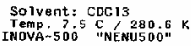

Relax. delay $1.000 \mathrm{sec}$

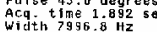

8 repet tions
OBSERVE
OHI

Totize 55596
Totame 0 min, 23 sec
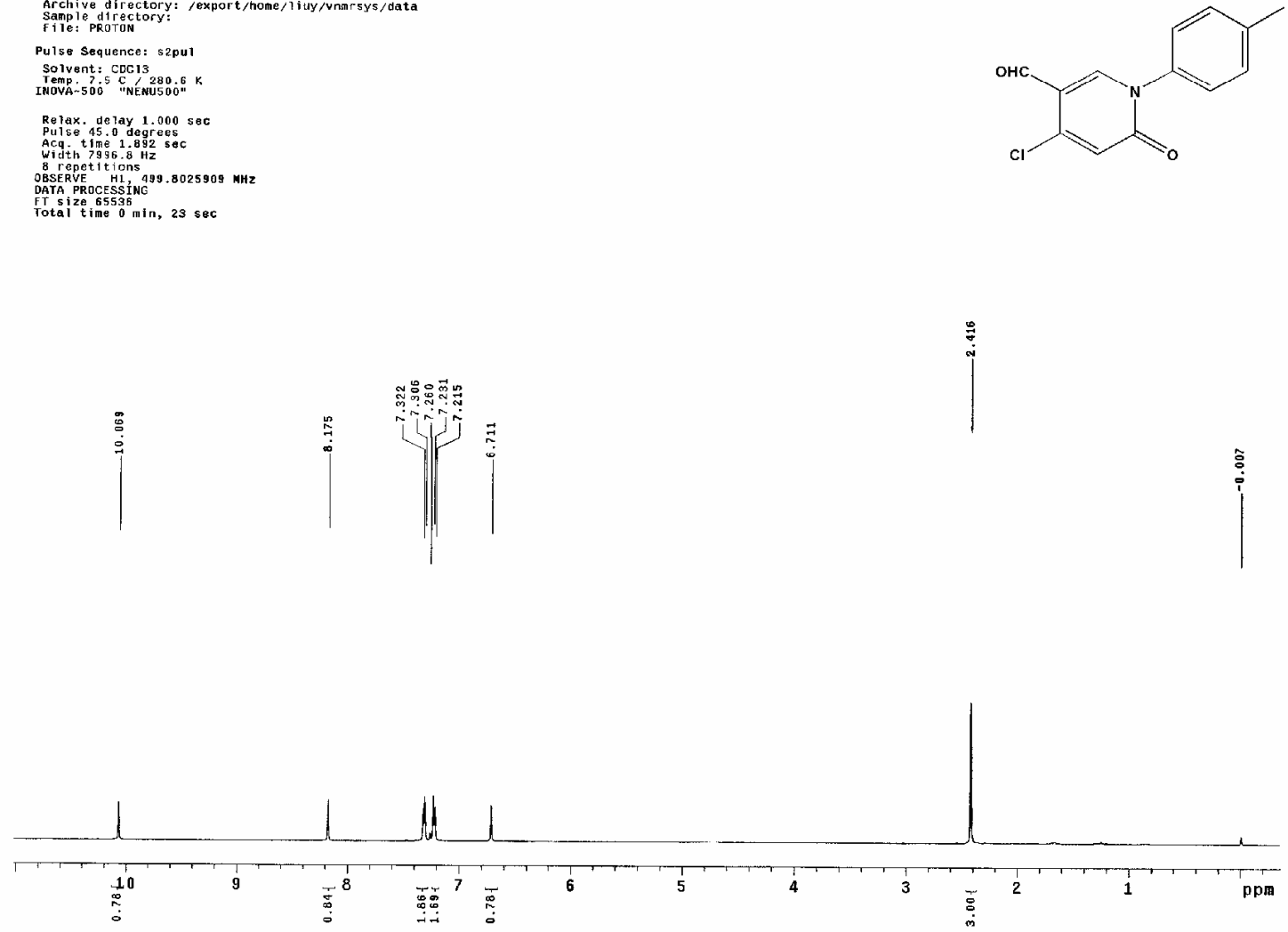

STANDARo Carbon Paraneters

Archive directory: /export/home/liuy/vnmrsys/data

Sample directory
file: CARBC

Pulse Sequence: szpur

Solvent: $\mathrm{CDC}^{2} 13$

USEI: 1-14-87 280.0 "NENU500"

Relax. delay $1.000 \mathrm{sec}$

Act time $1.300 \mathrm{sec}$

64 repetitions
OBSERV C13, $125.6754679 \mathrm{MHz}$

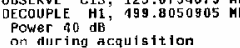

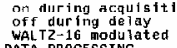

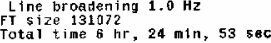
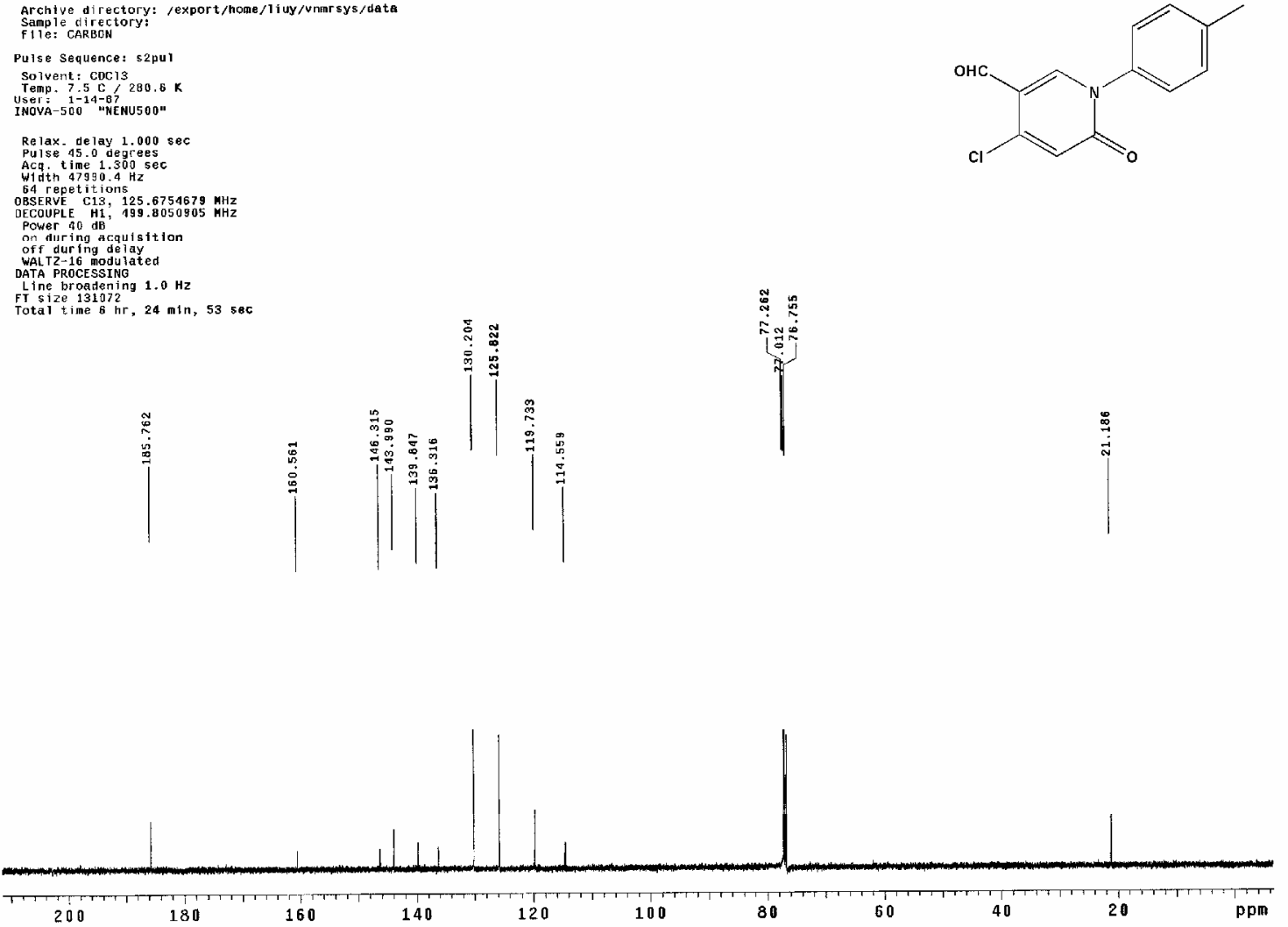
$3 q 2$

SIANDARD PROTON PARAMETERS

Archive directory: /export/home/1 i uy/vnmrsys/data
Sample directory:

Puse senuence

Solvent: cocis s2pul

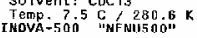

Relax. de lay $1.000 \mathrm{sec}$

Acq. time $1.892 \mathrm{sec}$

Widte $7996.8 \mathrm{~Hz}$

OBSERYE H1, 499.8025909 MHZ

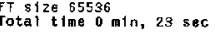
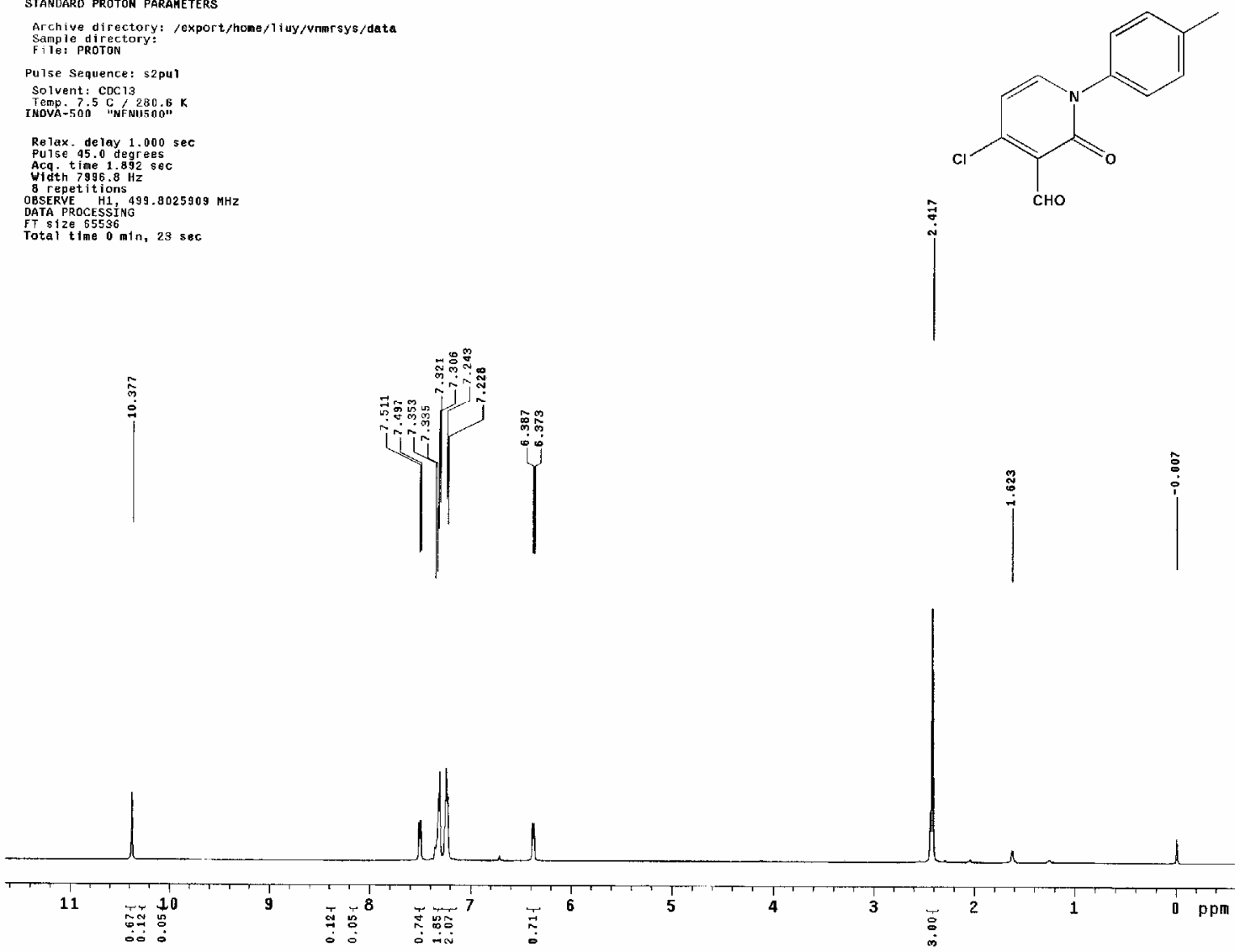

STANDARD CARBON PARAMETERS

Archive di rectory: /export/home/1i uy/vnmrsys/data
Sample directory:
File: CARBON

Pulse Sequence: s2pul

Solvent: coc 73 se.6 K

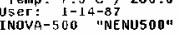

Relax. de lay 1,000 sec

Aca. time $1.300 \mathrm{sec}$

3264 repetitions
OBSERE C13; 125.6754905 MHZ
OECCUPLE HI; 499.8050905 HHZ

ow dur ing acquistetion

WAIT P-16 Dodulated

tine brobatening $1.0 \mathrm{~Hz}$

fT size 131072
Total time $3 \mathrm{hr}, 12 \mathrm{~min}, 26 \mathrm{sec}$
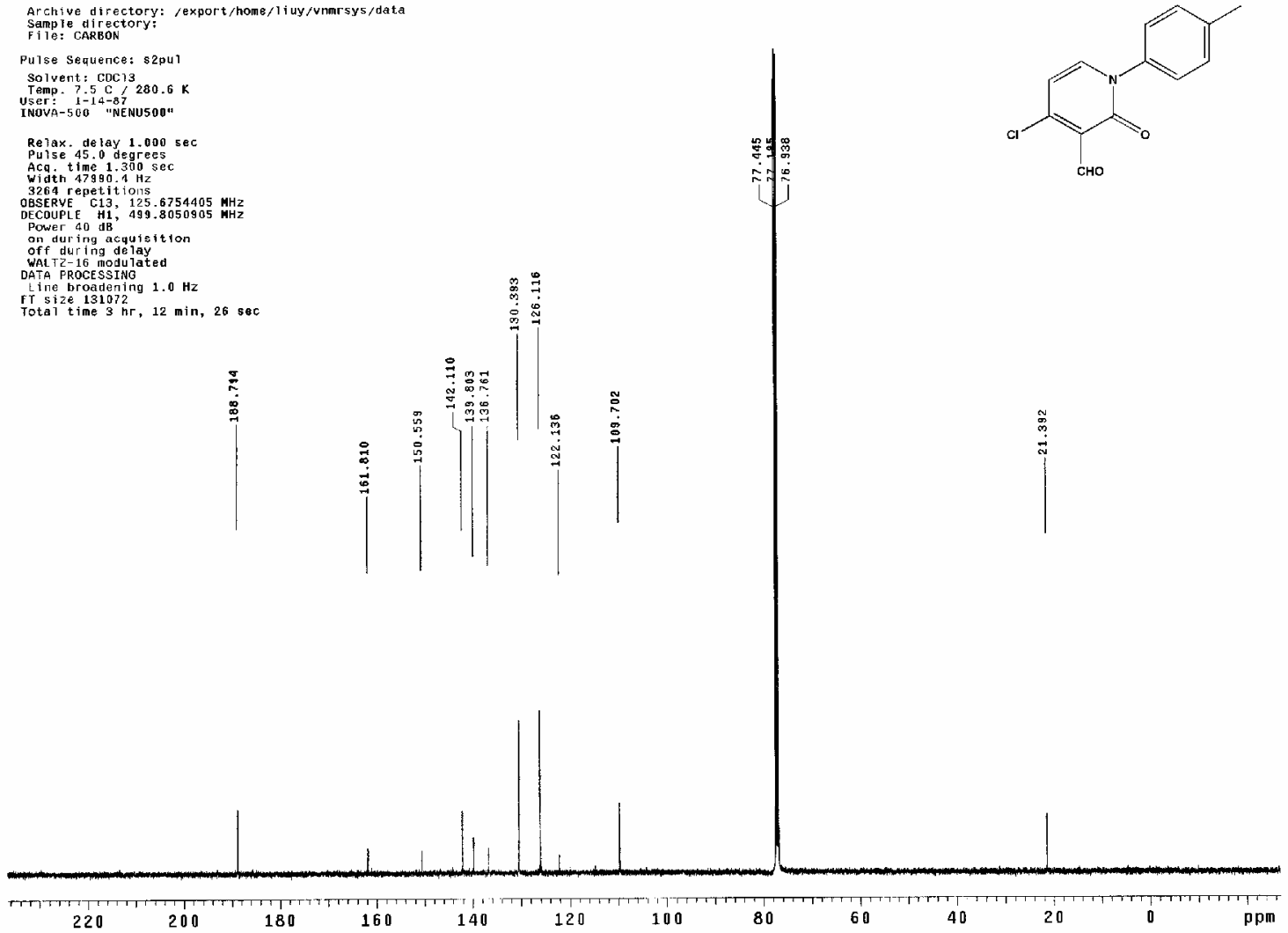
STANDARD PROTON PARAMETERS

Archive directory:
Sample directory:

Pulse sequence: $52 \mathrm{pu} 1$

Solvent: $\operatorname{coc} 13$

INOVA-500 "NEENSOOO"

Relax. de lay $1.000 \mathrm{sec}$
Pulse 45.0 degrees

Acq ${ }^{2}$ time 1.8925

OBSERVE H1, $199.8025915 \mathrm{MHZ}$

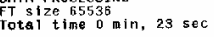
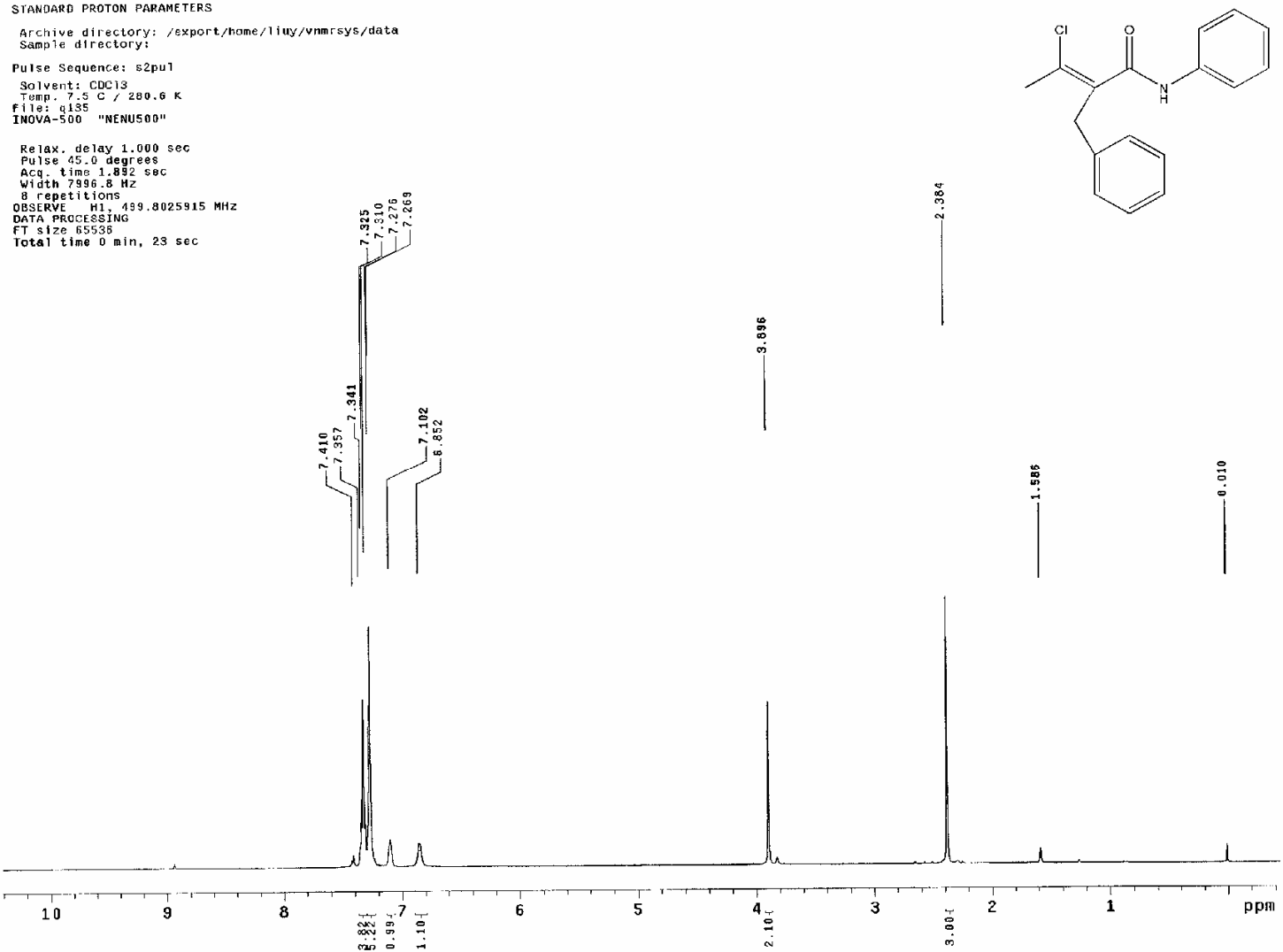

standard carbon parameters

Archive directory: /expart/home/ rituy/urmesys/date

Pulse Sequence: szpur

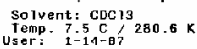

Relax. de lay 1.000 sec

Acy. time $1.300 \mathrm{sec}$

30 .
OBSERE C13, 125.6754327 HHZ
DECOUPLE H1, 499.8050905 MHZ

DECOUPLE $\mathrm{HL}^{\mathrm{H}}, 499.805092$
Power $40 \mathrm{~dB}$
on during acquisit

on during acquisit
off during delay
waLTZ-16 modulated
DATA PBOCESTHated

DiTA PRoCESSIIG

FT 5 i ze 131072
Total t $t$ me $6 \mathrm{hr}, 24 \mathrm{~min}, 53 \mathrm{sec}$

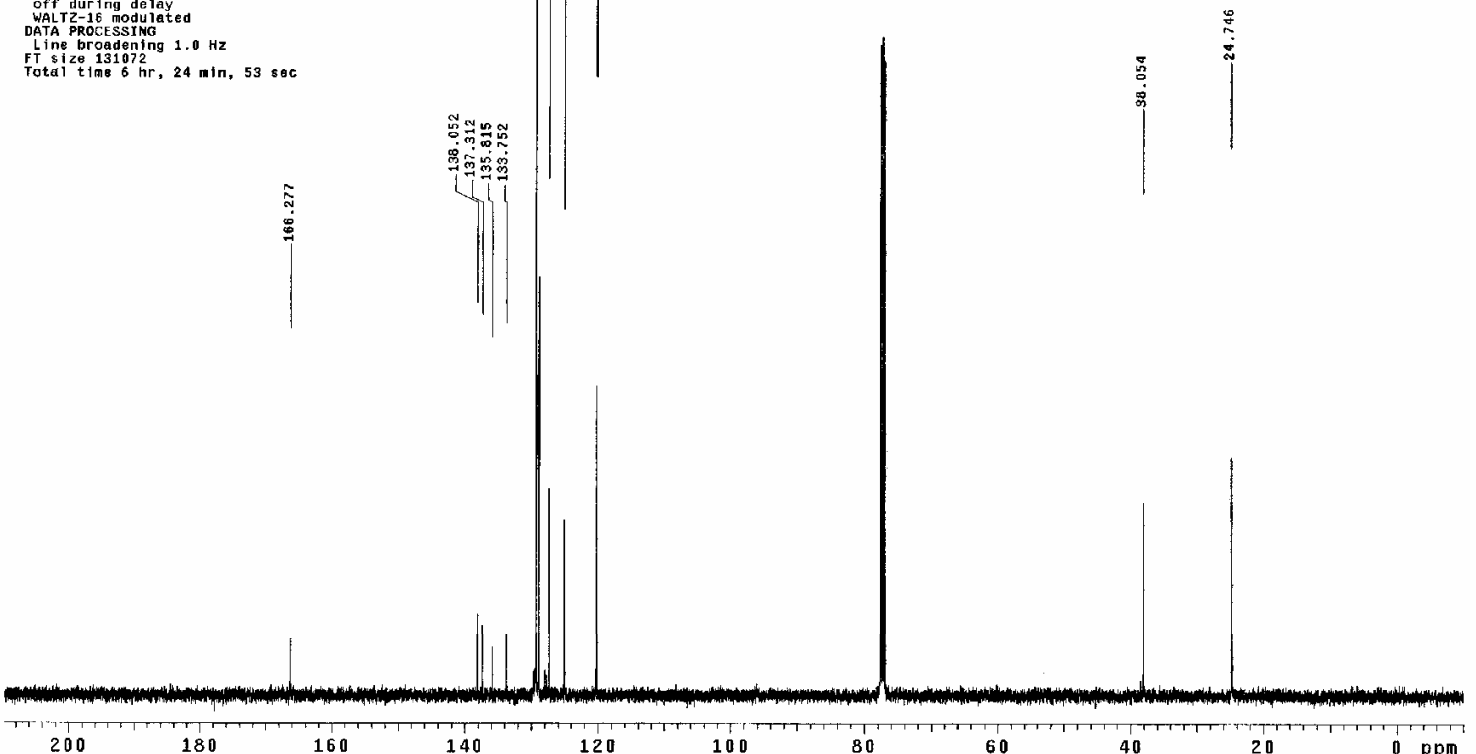

